\title{
Reaction-Diffusion Processes from Equivalent Integrable Quantum Chains
}

\author{
Malte Henkel ${ }^{a}$, Enzo Orlandini ${ }^{b, c}$ and Jaime Santos $^{b}$ \\ ${ }^{a}$ Laboratoire de Physique des Matériaux, Université Henri Poincaré Nancy I, BP 239, \\ F - 54506 Vandouvre-lès-Nancy Cedex, France, \\ ${ }^{b}$ Theoretical Physics, Department of Physics, \\ University of Oxford, 1 Keble Road, Oxford OX1 3NP, UK \\ ${ }^{c}$ Service de Physique Théorique, CEN Saclay, F- 91191 Gif-sur-Yvette Cedex, Francef
}

\begin{abstract}
One-dimensional reaction-diffusion systems are mapped through a similarity transformation onto integrable (and a priori non-stochastic) quantum chains. Time-dependent properties of these chemical models can then be found exactly. The reaction-diffusion processes related to free fermion systems with site-independent interactions are classified. The time-dependence of the mean particle density is calculated. Furthermore new integrable stochastic processes related to the Heisenberg XXZ chain are identified and the relaxation times for the particle density and density correlation for these systems are found.
\end{abstract}

PACS: 02.50.Ga, 05.40.+j, 82.20.Mj

\footnotetext{
${ }^{1}$ Unité de Recherche associée au CRNS no. 155

2 address after 1st october 1996
} 


\section{Introduction}

The physics of interacting particles out of thermodynamic equilibrium is an intriguing and challenging problem. For important reaction-diffusion processes which display a wide variety of interesting and unexpected phenomena, see [1, 2, 3, 团, 5] and references therein. They are not only of experimental importance but also pose a theoretical problem of considerable difficulty as there is no general framework for their treatment, such as detailed balance for equilibrium systems. While the classic approach, involving phenomenological rate equations, is sufficient to the describe the time-dependent behavior of non-equilibrium systems in sufficiently high spatial dimensions, in low dimensions the diffusive mixing is ineffective and new phenomena appear.

As an example, consider a system consisting of a single species of particles $A$ undergoing diffusion and subject to the two chemical reactions $A+A \rightarrow A$ with rate $r_{c}$ and $A+A \rightarrow$ inert with rate $r_{a}$. For this system one expects, starting from an initial particle density $\rho_{0}$, at late times an algebraic decay of the mean particle concentration [6]

$$
\rho_{A}(t) \sim \mathcal{A} t^{-y} \quad ; t \rightarrow \infty
$$

In one-dimensional systems, one has $y=\frac{1}{2}$ independently of the branching ratio $r_{c} / r_{a}$ as opposed to $y=1$ obtained from a (mean field) rate equation. The importance of fluctuations which invalidate the rate equation approach is underlined by recent experimental results for the exponent $y$ in effectively one-dimensional systems. In these experiments, the particles are excitons carried by long polymers and the effective one-dimensionality is achieved by either constraining the polymer to move in small pores of a convenient medium when the excitons move between the polymers [0, 专] or by the structure of the polymer itself when the excitons move along the polymer [9]. For mixed annihilation-coagulation (both $r_{a}, r_{c} \neq 0$ ) one finds $y=0.52-0.59$ [7] and $y=0.47(3)$ [8], while for the pure coagulation case $\left(r_{a}=0\right), y \simeq 0.48$ [9]. In the latter case, the numerical value of the exponent $y$ is also shown to be independent of the initial density $\rho_{0}$ [9], which confirms exponent universality. Furthermore, in that particular $1 D$ system, the amplitude $\mathcal{A}$ is independent of $\rho_{0}$ as well and it was realized [10 that this theoretical prediction is found experimentally [9] as well. This means that for low-dimensional systems with short-ranged interactions, simple kinetic equations as first investigated by Smoluchowski [11] are no longer adequate to describe the strong fluctuations occurring in these systems and leading to so-called anomalous kinetics [12]. More powerful techniques to include these effects must be sought.

Theoretical results of this nature can be obtained by a variety of means, e.g. the renormalization 
group (see e.g. [13]), improved mean-field approaches or exact calculations, see [2] for a collection of reviews. It is the aim of this paper to explore some of the possibilities of obtaining exact results. A convenient starting point for an exact description of a stochastic process is the formulation of the problem in terms of a master equation. The underlying assumption of this approach, viz. the absence of memory effects, is at least approximately true for many real systems. A master equation (see section 2 ) is a first order linear differential equation in time for the probability distribution of the stochastic variables describing the system. Using standard procedures, see [14, 16, 15, 17] it may be conveniently written as a matrix equation and thus its solution is turned into an eigenvalue problem?. The crucial point is that it has been realized in recent years that for many models of interest the matrix appearing in this eigenvalue problem is the quantum Hamiltonian (or the transfer matrix respectively, in the case of discrete time dynamics) of some integrable quantum chain [18, 19, 20, 21, 22, 23, 24, 25, 26, 27, 28, 29, 30, 31, 32, 33, 34. This insight has made available the tool box of integrable models (e.g. Bethe ansatz [35, 36, 37]) for these interacting particle systems far from equilibrium and has led to many new exact results for their dynamical and stationary properties. Clearly, the models tractable by such means are usually rather simple, but the observed universality of many quantities of interest make these models both theoretically and experimentally interesting.

Most integrable stochastic models studied so far are directly given in terms of an integrable quantum chain or transfer matrix. In these cases, "just by looking" at the problem one recognizes some known integrable system. However, it has been observed recently that one may obtain from a given stochastic quantum Hamiltonian by a similarity transformation the Hamiltonian of some other stochastic process, see [38, 39, 10, 40]. This relates a system which is not obviously integrable to another system which is and in this way exact results may be obtained. At the same time it was also realized that indeed one may obtain by a similarity transformation a stochastic Hamiltonian from some integrable Hamiltonian which does not describe a stochastic process [25, 10]. This is implicit in many earlier treatments of reaction-diffusion processes, but has not yet been exploited systematically. It shows that many more stochastic systems than previously thought are actually integrable.

In this paper, our strategy is as follows. We start from some (a priori non-stochastic) integrable quantum chain $H$. The examples we shall consider can be analyzed using Bethe ansatz and/or free fermion techniques. Next we perform a similarity transformation and ask under which conditions on the parameters of the original integrable quantum chain $H$ and on the transformation matrix one obtains

\footnotetext{
${ }^{3}$ For models with discrete-time dynamics one obtains an eigenvalue problem relating the probability distribution of the system at time $t=n+1$ to the distribution at time $t=n$
} 
a stochastic Hamiltonian. In this way we identify several classes of new integrable stochastic models. Finally, we use the knowledge provided by the integrability to obtain some exact results for these processes, in particular, the average density at time $t$, and the (longest) relaxation time in the system.

Pursuing this program in full generality appears to be a formidable task. Therefore we shall restrict ourselves to systems which are (a) translationally invariant, (b) involve particles of only one kind with hard core repulsion preventing double occupancy of a single site and (c) which interact only via effective nearest neighbor interaction. We shall make at various points comments on generalizations, e.g. to other boundary conditions, but we shall not work out any details in these cases. Finally, we shall consider only similarity transformations which are translationally invariant and which preserve the locality of all observables. This excludes e.g. sublattice transformations [41] or duality transformations 42, 43] which also give rise to transformations between different stochastic Hamiltonians or between non-stochastic and stochastic Hamiltonians. We would like to stress that we shall consider only continous-time dynamics. However, all our results apply also to models with suitably chosen discrete time dynamics.

The paper is organized as follows: in section 2 we review the Hamiltonian formulation for nonequilibrium stochastic systems and its connection with integrable quantum Hamiltonians of magnetic systems. We also specify the similarity transformations which will be used to link stochastic systems with integrable ones and comment briefly on the relationship with continuum field theory. In section 3, we give the full classification of the stochastic systems which can be reduced to a free fermion Hamiltonian with site-independent interactions. In section 4, we find stochastic systems which have the same spectrum as a XXZ chain with real matrix elements. In section 5, we use the link with the free fermion case to calculate explicitly the time-dependent particle density. Section 6 gives our conclusions. More technical material is relegated to the appendices. In appendix A, we extended the standard technique of Lieb-Schultz-Mattis for the diagonalization of a hermitian free fermion Hamiltonian to the non-hermitian case. Appendix B gives further details for the classification of those stochastic systems similar to a free fermion Hamiltonian. In appendix $\mathrm{C}$ we recall the relationship of one of the stochastic systems related to free fermions to the biased voter model. Appendix D gives some further details for the construction of stochastic processes from the XXZ chain. Appendix E describes the details of the caculation of the long-time behaviour of particle densities and two-point correlators from both the Bethe ansatz and the truncated equations of motion. Appendix F recalls the relationship between the Hamiltonian spectra for periodic and free boundary conditions. 


\section{Quantum chain formulation of stochastic processes}

In this section, we review the reformulation of a non-equilibrium stochastic system defined by some master equation in terms of the spectral properties of an associated quantum Hamiltonian $H$. To be specific, we only consider systems defined on a chain with $L$ sites and two allowed states per site. We represent the states of the system in terms of spin configurations $\{\sigma\}=\left\{\sigma_{1}, \sigma_{2}, \ldots, \sigma_{L}\right\}$ where $\sigma=+1$ corresponds to an empty site and $\sigma=-1$ corresponds to a site occupied by a single particle. We are interested in the probability distribution function $P(\sigma ; t)$ of the configurations $\{\sigma\}$. Our starting point is the master equation for the $P(\sigma ; t)$

$$
\partial_{t} P(\sigma ; t)=\sum_{\tau \neq \sigma}[w(\tau \rightarrow \sigma) P(\tau ; t)-w(\sigma \rightarrow \tau) P(\sigma ; t)]
$$

where $w(\tau \rightarrow \sigma)$ are the transition rates between the configurations $\{\tau\}$ and $\{\sigma\}$ and are assumed to be given from the phenomenology. In order to rewrite this as a matrix problem, we introduce a state vector

$$
|P\rangle=\sum_{\sigma} P(\sigma ; t)|\sigma\rangle
$$

and eq. 2.1) becomes

$$
\partial_{t}|P\rangle=-H|P\rangle
$$

where the matrix elements of $H$ are given by

$$
\langle\sigma|H| \tau\rangle=-w(\tau \rightarrow \sigma) \text { if } \tau \neq \sigma \quad,\langle\sigma|H| \sigma\rangle=\sum_{\tau \neq \sigma} w(\sigma \rightarrow \tau)
$$

The operator $H$ describes a stochastic process since all the elements of the columns add up to zero. This conservation of probability, viz. $\sum_{\sigma} P(\sigma ; t)=1$, is equivalent to the relation

$$
\langle s| H=0
$$

where $\langle s|=\sum_{\sigma}\langle\sigma|$ is a left eigenvector of $H$ with eigenvalue 0 . Correspondingly, $H$ has at least one right eigenvector with eigenvalue 0 . Such a vector does not evolve in time and therefore corresponds to a steady state distribution of the system. Since in general $H$ is not symmetric, this steady state vector may be highly non-trivial. Note that all this is completely general and applies to any stochastic process defined by a master equation. With a view on the processes that we shall study we call $H$ a quantum Hamiltonian and this formulation of the master equation the quantum Hamiltonian formalism. The reason for this choice of language is the fact that for the processes studied below (and, in fact, many 
other processes as well) $H$ is the Hamiltonian of some quantum system such as the Heisenberg $X X Z$ Hamiltonian. The steady state of a stochastic system corresponds in this mapping to the ground state of the quantum system.

Now, it is straightforward to translate the well-known theorems [44] about the solution of the master equation (2.1) to the Hamiltonian formulation at hand. In particular, the real parts of the eigenvalues $E_{i}$ of $H$ are non-negative, $\Re E_{i} \geq E_{0}=0$, and there are no purely imaginary eigenvalues, which excludes the possibility of undamped oscillations. Furthermore, if the initial distribution $P_{0}(\{\sigma\})=P(\sigma ; 0)=\left\langle\sigma \mid P_{0}\right\rangle$ satisfies $0 \leq P_{0}(\{\sigma\}) \leq 1$ and $\left\langle s \mid P_{0}\right\rangle=1$, it follows that since

$$
|P\rangle=\exp (-H t)\left|P_{0}\right\rangle
$$

the relations $0 \leq\langle\sigma \mid P\rangle \leq 1$ and $\langle s \mid P\rangle=1$ hold for all times $t$. This guarantees the probabilistic interpretation. Then time-dependent averages of an observable $F$ are given by the matrix element

$$
<F>(t)=\sum_{\sigma} F(\sigma) P(\sigma ; t)=\langle s|F| P\rangle=\left\langle s|F \exp (-H t)| P_{0}\right\rangle
$$

Conversely, if a matrix $\widetilde{H}$ satisfies the conditions

$$
\langle s| \widetilde{H}=0,\langle\sigma|\widetilde{H}| \tau\rangle \leq 0 \text { for all } \sigma \neq \tau
$$

it follows that $\widetilde{H}$ is the generator of a stochastic process 44 . These conditions will play a major role later on. We shall refer to the first as probability conservation condition and to the second as positivity conditions.

In what follows, we shall be mainly interested in averages of particle numbers $n_{j}$ at site $j$ and their correlators. These can be expressed in the quantum spin formulation in terms of the projector

$$
\tilde{n}_{j}=\frac{1}{2}\left(1-\sigma_{j}^{z}\right)=\left(\begin{array}{ll}
0 & 0 \\
0 & 1
\end{array}\right)_{j}
$$

and one-point and two-point functions of the $n_{j}$ are then expressed as 1

$$
<\tilde{n}_{j}>(t)=\left\langle s\left|\tilde{n}_{j}\right| P\right\rangle, \quad<\tilde{n}_{j} \tilde{n}_{\ell}>(t)=\left\langle s\left|\tilde{n}_{j} \tilde{n}_{\ell}\right| P\right\rangle
$$

Two basic situations are readily distinguished from the spectrum of $H$. If in the limit of infinite lattice size $L \rightarrow \infty$ the lowest excited states have a finite gap to the ground state energy $E_{0}=0$, then the averages (2.10) will approach their steady state values exponentially fast with time. On the other

\footnotetext{
${ }^{4}$ We stress that the structure of these matrix elements is quite distinct from expectation values in ordinary quantum mechanics where one always considers matrix elements between right and left states that are hermitean conjugates.
} 
hand, if there is in the $L \rightarrow \infty$ limit a continuous spectrum down to $E_{0}=0$, one expects an algebraic approach of the correlators to the steady state.

The recent interest in this setup comes from the integrability of quantum Hamiltonians in one dimension [19, 21, 23, 24, 25, 26, 27]. As the paradigmatic example, consider the asymmetric simple exclusion process where particles are diffusing to the right, $A+\emptyset \rightarrow \emptyset+A$ with rate $1 / q$ and diffusing to the left $\emptyset+A \rightarrow A+\emptyset$ with rate $q$. The Hamiltonian generating the time evolution of the system can, for free boundary conditions, be written in the form [24]

$$
H=-\frac{1}{2} \sum_{j=1}^{L-1}\left[\sigma_{j}^{x} \sigma_{j+1}^{x}+\sigma_{j}^{y} \sigma_{j+1}^{y}+\frac{1}{2}\left(q+q^{-1}\right)\left(\sigma_{j}^{z} \sigma_{j+1}^{z}-1\right)-\frac{1}{2}\left(q-q^{-1}\right)\left(\sigma_{j}^{z}-\sigma_{j+1}^{z}\right)\right]
$$

where $\sigma^{x, y, z}$ are Pauli matrices. Through various mappings this model arises in a variety of contexts, it is related e.g. to the $1 D$ Kardar-Parisi-Zhang equation or to the noisy Burgers equation, see e.g. [4] for a review. $H$ can be analysed exactly using the Bethe ansatz [21]. It is identical to the integrable quantum group $U_{q} S U(2)$ Hamiltonian 45, 46] and gives a clear physical interpretation to the quantum deformation parameter $q$ as hopping asymmetry [24]. The quantum group symmetry may be used for the calculation of correlation functions [28]. It is well-known that the spectrum of $H$ has a finite gap for $q \neq 1$. For $q=1$ however, corresponding to symmetric diffusion, the energy levels scale with the system size $E_{i} \sim L^{-2}$. In contrast to conventional equilibrium systems, the spectral properties of $H$ depend on the boundary conditions. For periodic boundary conditions, it can be shown that for all values of $q$, there is no gap for the infinite system and $E_{i} \sim L^{-\theta}$, where $\theta$ varies between $\frac{3}{2}$ and 2 depending on the particle density [21, 47, 27]. The basic mechanism behind this is explained in appendix F.

In fact, integrable quantum Hamiltonians can be found for much more general systems. We restrict our attention here to models with nearest-neighbor interactions and only consider a single species of particles. Unfortunately, there is no standard notation for the various rates. In table 11 we give the possible reaction-diffusion processes together with their rates, providing at the same time a dictionary between the notations used by different authors. In this paper, we shall use the notation employed in [10].

For the time being and for purposes of illustration let us consider besides diffusion only those reactions which irreversibly reduce the number of particles (that is, $\beta_{L, R}=\sigma_{L, R}=\nu=0$ ). For temporary convenience we rescale $\alpha \rightarrow D \alpha$ and define also

$$
D=\sqrt{D_{L} D_{R}}, \gamma=\frac{\sqrt{\gamma_{L} \gamma_{R}}}{D}, \delta=\frac{\sqrt{\delta_{L} \delta_{R}}}{D}, q=\sqrt{\frac{D_{L}}{D_{R}}}=\sqrt{\frac{\gamma_{L}}{\gamma_{R}}}=\sqrt{\frac{\delta_{L}}{\delta_{R}}}
$$

\footnotetext{
${ }^{5}$ Or equally, from the integrability of transfer matrices for discrete time dynamics [20, 22]
} 


\begin{tabular}{|l|c|c|c|c|c|}
\hline diffusion to the left & $\emptyset+A \rightarrow A+\emptyset$ & $D_{L}$ & $a_{32}$ & $w_{1,1}(1,0)$ & $\Gamma_{10}^{01}$ \\
diffusion to the right & $A+\emptyset \rightarrow \emptyset+A$ & $D_{R}$ & $a_{23}$ & $w_{1,1}(0,1)$ & $\Gamma_{01}^{10}$ \\
pair annihilation & $A+A \rightarrow \emptyset+\emptyset$ & $2 \alpha$ & $a_{14}$ & $w_{1,1}(0,0)$ & $\Gamma_{00}^{11}$ \\
coagulation to the right & $A+A \rightarrow \emptyset+A$ & $\gamma_{R}$ & $a_{24}$ & $w_{1,0}(0,1)$ & $\Gamma_{01}^{11}$ \\
coagulation to the left & $A+A \rightarrow A+\emptyset$ & $\gamma_{L}$ & $a_{34}$ & $w_{0,1}(1,0)$ & $\Gamma_{10}^{11}$ \\
death at the left & $A+\emptyset \rightarrow \emptyset+\emptyset$ & $\delta_{L}$ & $a_{13}$ & $w_{1,0}(0,0)$ & $\Gamma_{00}^{10}$ \\
death at the right & $\emptyset+A \rightarrow \emptyset+\emptyset$ & $\delta_{R}$ & $a_{12}$ & $w_{0,1}(0,0)$ & $\Gamma_{00}^{01}$ \\
decoagulation to the left & $\emptyset+A \rightarrow A+A$ & $\beta_{L}$ & $a_{42}$ & $w_{1,0}(1,1)$ & $\Gamma_{11}^{01}$ \\
decoagulation to the right & $A+\emptyset \rightarrow A+A$ & $\beta_{R}$ & $a_{43}$ & $w_{0,1}(1,1)$ & $\Gamma_{11}^{10}$ \\
birth & $\emptyset+\emptyset \rightarrow A+A$ & $2 \nu$ & $a_{41}$ & $w_{1,1}(1,1)$ & $\Gamma_{11}^{00}$ \\
creation at the right & $\emptyset+\emptyset \rightarrow \emptyset+A$ & $\sigma_{R}$ & $a_{21}$ & $w_{0,1}(0,1)$ & $\Gamma_{01}^{00}$ \\
creation at the left & $\emptyset+\emptyset \rightarrow A+\emptyset$ & $\sigma_{L}$ & $a_{31}$ & $w_{1,0}(1,0)$ & $\Gamma_{10}^{00}$ \\
\hline Rates defined according to reference & {$[10$} & {$[25]$} & {$[24$} & {$[48$} \\
\hline
\end{tabular}

Table 1: Two-sites reaction-diffusion processes and their rates as defined by various authors.

$$
\Delta=\frac{1}{2}\left(q+q^{-1}\right)(1+\delta-\gamma)-\alpha, \quad h=\frac{1}{2}\left(2 \alpha+\gamma\left(q+q^{-1}\right)\right) .
$$

Note that the ratio of the left and right rates is taken to be the same for diffusion, coagulation and death processes. Then the quantum Hamiltonian for the system may be written in the form

$$
H=D\left(H_{X X Z}(h, \Delta, \delta)+H_{\alpha}+H_{\gamma}+H_{\delta}\right)
$$

where $H_{X X Z}(h, \Delta, \delta)$ is the standard $X X Z$ quantum chain, written down again for free boundary conditions

$$
\begin{aligned}
H_{X X Z}(h, \Delta, \delta) & =-\frac{1}{2} \sum_{j=1}^{L-1}\left[\sigma_{j}^{x} \sigma_{j+1}^{x}+\sigma_{j}^{y} \sigma_{j+1}^{y}+\Delta\left(\sigma_{j}^{z} \sigma_{j+1}^{z}-1\right)\right. \\
& \left.+h\left(\sigma_{j}^{z}+\sigma_{j+1}^{z}-2\right)-\frac{1}{2}(1-\delta)\left(q-q^{-1}\right)\left(\sigma_{j}^{z}-\sigma_{j+1}^{z}\right)\right]
\end{aligned}
$$

which contains the diagonal and diffusive matrix elements while the particle annihilation terms are contained in

$$
\begin{aligned}
& H_{\alpha}=-2 \alpha \sum_{j=1}^{L-1} q^{-2 j-1} \sigma_{j}^{+} \sigma_{j+1}^{+} \\
& H_{\gamma}=-\gamma \sum_{j=1}^{L-1} q^{-j}\left(\tilde{n}_{j} \sigma_{j+1}^{+}+q^{-1} \sigma_{j}^{+} \tilde{n}_{j+1}\right) \\
& H_{\delta}=-\delta \sum_{j=1}^{L-1} q^{-j}\left(q^{-2}\left(1-\tilde{n}_{j}\right) \sigma_{j+1}^{+}+q \sigma_{j}^{+}\left(1-\tilde{n}_{j+1}\right)\right)
\end{aligned}
$$

and $\sigma^{ \pm}=\frac{1}{2}\left(\sigma^{x} \pm i \sigma^{y}\right)$ are the one-particle annihilation/creation operators. 
Remarkably, the spectrum of $H$ is independent of the terms contained in (2.16), that is $\operatorname{spec}(H)=$ $\operatorname{spec}\left(D H_{X X Z}(h, \Delta, \delta)\right)$ [24]. To see this, recall that the XXZ Hamiltonian conserves the number of particles while the reaction terms irreversibly decrease the total particle number. Thus, $H$ can be written in a block diagonal form

$$
H=\left(\begin{array}{ccccc}
\mathcal{N}_{0} & X_{\delta} & X_{\alpha} & & \\
& \mathcal{N}_{1} & X_{\gamma, \delta} & X_{\alpha} & \\
& & \mathcal{N}_{2} & X_{\gamma, \delta} & \ddots \\
& & & \ddots & \ddots
\end{array}\right)
$$

where $\mathcal{N}_{n}$ refers to the $n$-particle states and $X$ are the reaction matrix elements. Because of the identity

$$
\operatorname{det}\left(\begin{array}{cc}
\mathcal{A} & X \\
0 & \mathcal{B}
\end{array}\right)=\operatorname{det} \mathcal{A} \operatorname{det} \mathcal{B}
$$

it is clear that the elements of (2.16) do not enter into the characteristic polynomial of $H$.

The phase diagram for $H_{X X Z}(h, \Delta, \delta)$ is known [49, 50]. For our purposes, we need the following. From (2.13), only the portion of the phase diagram where $h+\Delta \geq 1$ is important for us. The spectrum always has a finite gap when $h+\Delta>1$, which is realized whenever $\delta \neq 0$ or $q \neq 1$. Then the ground state is a trivial ferromagnetic frozen state. The spectrum is gapless for $\Delta+h=1$, where the system undergoes a Pokrovsky-Talapov transition. This situation occurs for $\delta=0$ and $q=1$. We have thus identified the cases where the model approaches the steady state exponentially (non-vanishing gap) or algebraically (gapless). A special point is $\Delta=0$ where $H_{X X Z}$ becomes a free fermion system and thus in certain cases not only the spectrum may be obtained, but also correlation functions can be calculated explicitly (see below).

While large classes of reaction-diffusion problems are now recognised as being integrable, see [23, 25, 26] for lists including systems with more than one kind of particles 7 , it is still far from obvious how to exploit the algebraic structure hidden beneath it in order to get explicit expressions for the desired particle number correlators for $\Delta \neq 0$. A very interesting alternative was proposed in [48], where subsets of observables were identified for which closed equation of motion can be obtained, thus leading to partially integrable systems. In any case, it is important to realize that the knowledge about the spectrum (and, in certain cases such as the free fermion case $\Delta=0$, about correlators) does not

\footnotetext{
${ }^{6}$ Alternatively, this can also be seen through a similarity transformation of the form (2.19), where $B$ is diagonal. While this does not change $H_{X X Z}$, the rates occurring in (2.16) become arbitrary, since they contain the elements of $B$. Thus $\operatorname{spec}(H)$ must be independent of them 24 .

${ }^{7}$ We remark that up to degeneracy, the spectrum of $H$ for the processes $A+\emptyset \leftrightarrow \emptyset+A, B+\emptyset \leftrightarrow \emptyset+B$ with rate 1 , $A+A \rightarrow B, B+B \rightarrow A$ with rate $\alpha$ and $A+B \rightarrow \emptyset+\emptyset$ with rate $2 \alpha$, is the one of $H_{X X Z}(\alpha, 1-\alpha, 0)$ [24]. For $\alpha=1$, one recovers a free fermion system and closed equations of motion can be found and solved [51, leading for example to $\rho_{A}(t) \sim \rho_{B}(t) \sim 1 / \sqrt{t}$.
} 
come from the assumption that the Hamiltonian is stochastic, but from its integrability. It is therefore natural to ask whether one can find new integrable non-equilibrium systems from transformations of known integrable quantum chains.

Specifically, we shall investigate the transformation 38, 39, 10]

$$
\widetilde{H}=\mathcal{B H B}^{-1}, \mathcal{B}=\bigotimes_{j=1}^{L} B_{j}
$$

where $H$ is a quantum Hamiltonian with known properties, $\widetilde{H}$ a stochastic Hamiltonian and $B_{j}$ is the transformation matrix $B$ acting on the site $j$. From now on, we shall focus on translationally invariant systems, i.e. we shall consider periodic boundary conditions. To study the effect of the transformation $\mathcal{B}$, it is sufficient to consider the effect on a two-particle Hamiltonian $H_{j, j+1}$, that is we write

$$
H=\sum_{j=1}^{L} \mathbf{1}_{1} \otimes \cdots \otimes \mathbf{1}_{j-1} \otimes H_{j, j+1} \otimes \mathbf{1}_{j+2} \cdots \otimes \mathbf{1}_{L}
$$

where $\mathbf{1}_{\ell}$ is the unit matrix acting on the site $\ell$. For the model (2.14) with left-right symmetry, that is $q=1$, we have for example

$$
H_{j, j+1}=D\left(\begin{array}{cccc}
0 & -\delta & -\delta & -2 \alpha \\
0 & 1+\delta & -1 & -\gamma \\
0 & -1 & 1+\delta & -\gamma \\
0 & 0 & 0 & 2(\alpha+\gamma)
\end{array}\right)
$$

Transformations of the type (2.19) have been mainly studied when both $H$ and $\widetilde{H}$ represent stochastic systems. Besides, an equivalent way of relating stochastic systems was developed [52] in the context of the Lagrangian formulation of the model (see below), which is more suitable for field theory techniques. In particular, these transformations allow to treat the general model (2.21), since the observables in the two systems are simply related [10, 38, 39, 40, 52. The results for the long-time behaviour of the oneand two-point functions for translationally invariant initial conditions are collected in table 2. For the derivation, see appendix E.

From the spectrum of $H$, we would naively expect exponential factors $e^{-2 k \delta t}$ to be present in the $k$-point correlator $C_{k}$, but we also see that for example algebraic prefactors are not readily predicted from the spectrum alone. This expectation, however, is only valid provided there are no bound states in the system. This is so as long the model stays sufficiently close to the case $\delta=\alpha+\gamma-1$, where the exact spectrum of $H$ can be found from free fermion techniques. In general, however, one can show from the Bethe ansatz [53, 35, 36, 37] that there does exist a bound state in the two-particle sector, which has the energy $4 \delta+4-2 \Delta-2 / \Delta$. While the results given in table 2 are valid for homogeneous initial

\footnotetext{
${ }^{8}$ We thank G.M. Schütz for a useful discussion on this point.
} 


\begin{tabular}{|c|cc|}
\hline$\delta$ & $C_{1}$ & $C_{2}$ \\
\hline 0 & $t^{-1 / 2}$ & $t^{-3 / 2}$ \\
$<\alpha+\gamma$ & $\exp (-2 \delta t)$ & $t^{-1 / 2} \exp (-4 \delta t)$ \\
$>\alpha+\gamma$ & $\exp (-2 \delta t)$ & $\exp [-4 \delta t+2(\Delta+1 / \Delta-2) t]$ \\
\hline
\end{tabular}

Table 2: Generic long time behaviour of the one-point function $\left.C_{1}(t)=\sum_{j}<n_{j}\right\rangle(t)$ and the two-point function $C_{2}(t)=\sum_{j}\left\langle n_{j} n_{j+r}\right\rangle(t)$ in the system eq. (2.21) for $r$ finite, translation invariant initial conditions and finite initial particle density. The abbreviation $\Delta=1+\delta-\alpha-\gamma$ is used .

conditions, further power law prefactors may appear for other types of initial conditions (an example of this will be discussed in section 5).

A few comments are in order. When $\alpha, \gamma$ are arbitrary and $\delta=0$, the model can be reduced [39, 10] to the cases $\gamma=0$ or $\alpha=0$ which had so far been the only ones amenable to exact treatment, see [54, 55]. Thus the particle number correlators can be found exactly for all times. An equivalent transformation was subsequently given in the continuum field theory formulation of the theory [52]. For $\delta \neq 0$, the same technique allows the transformation to a system which can be interpreted as describing radioactively decaying particles $\square$ which in addition undergo diffusion and either annihilation or death processes. Then it can be shown that in the long-time limit the equations of motion for the $n$-point functions decouple and the long-time behaviour of the correlators can be extracted [10]. Furthermore, for left-right nonsymmetric rates $(q \neq 1)$ by the same reduction technique it can be shown that for $\delta_{L, R}=0$, the coagulation rates $\gamma_{L, R}$ can be eliminated if and only if the free-fermion condition is satisfied [10] and the correlators are then again known exactly for all times.

Here, we concentrate on transformations between an integrable, but not necessarily stochastic, quantum chain $H$ and a stochastic Hamiltonian $\widetilde{H}$. Some examples of this have already been given [38, 10]. We attempt to treat the transformation of type (2.19) systematically starting from two types of an integrable quantum chain

1. The most general translationally invariant Hamiltonian which be may written in terms of free

\footnotetext{
${ }^{9}$ The above analysis assumes that the associated amplitudes are non-vanishing. This is not always so, as can be seen in the calculation of the correlator $\left\langle n_{j}(t) n_{\ell}(0)>\right.$ for a model in which the only non-vanishing rates are $\alpha$ and $\nu$. Although a bound state is known to be present, the associated amplitude can be shown to vanish. We thank R. B. Stinchcombe for pointing this out to us.

${ }^{10}$ The radioactive decay of particles is described in this setting by the conditions $\alpha=0$ and $\gamma=\delta$.

${ }^{11}$ We limit ourselves to these two types of integrable systems for pragmatic reasons. In fact, for the second type of integrable system we consider here we merely use the integrability of the spectrum and do not even ask whether or not the entire system might be integrable. A sufficient criterion to establish integrability of the full Hamiltonian $H$ is provided by showing that the $H_{j, j+1}$ satisfy a Hecke algebra. It can be shown that for $\delta=0$ in the model (2.14) the entire non-symmetric Hamiltonian matrix and not just the spectrum-determining part is integrable [24]. See [23] for a list of integrable stochastic Hamiltonians through the Hecke algebra technique.
} 
fermions. We shall give in the next section the complete list of stochastic systems with spatially constant reaction rates which can be transformed into this form.

2. The most general Hamiltonian with the spectrum of the $X X Z$ chain and with real matrix elements. Here we shall obtain a new 3-parameter manifold (after normalization) of integrable stochastic processes.

Before starting this, however, it might be useful to recall briefly how results obtained from the quantum Hamiltonian formulation of reaction-diffusion systems presented here compare with the Lagrangian techniques, as developed in [15, 17, 56]. See [13] for a recent review. As for the quantum Hamiltonian formulation, the starting point is the master equation. The creation and destruction of particles is conveniently described in a Fock space formalism, where the state vector becomes

$$
|P(t)\rangle=\sum_{\sigma} p\left(n_{1}, n_{2}, \ldots ; t\right)\left(a_{1}^{\dagger}\right)^{n_{1}}\left(a_{2}^{\dagger}\right)^{n_{2}} \cdots|0\rangle
$$

where $a_{i}^{\dagger}$ creates a particle at site $i$ and $p\left(n_{1}, n_{2}, \ldots ; t\right)$ is the probability of having $n_{1}$ particles at site $1, n_{2}$ particles at site 2 and so on at time $t$. Consider for example the case when only diffusion and annihilation occur. Then the Hamiltonian is

$$
H=D \sum_{(i, j)}\left(a_{i}^{\dagger}-a_{j}^{\dagger}\right)\left(a_{i}-a_{j}\right)-2 \alpha \sum_{i}\left(a_{i}^{2}-a_{i}^{\dagger^{2}} a_{i}^{2}\right) \rightarrow \int\left[D\left(\nabla a^{\dagger}\right)(\nabla a)-2 \alpha\left(a^{2}-a^{\dagger^{2}} a^{2}\right)\right] d^{d} x
$$

where a formal continuum limit is taken $\square$. One then goes over to a path integral representation involving fields $a(x, t)$ and $a^{*}(x, t)$ and an action $S\left[a, a^{*}\right]$. In order to reduce the calculation of avarages to the usual QFT expectation values, it is customary to perform a shift $a^{*}=1+\bar{a}$. Then the action becomes [15, 17, 56]

$$
S[a, \bar{a}]=\int\left[\bar{a} \partial_{t} a+D \nabla \bar{a} \nabla a+4 \alpha \bar{a} a^{2}+2 \alpha \bar{a}^{2} a^{2}\right] d t d^{d} x
$$

Remarkably, since the particle number can only decrease, the diagrammatics of the associated field theory is greatly simplified. In particular, there are no loop corrections to the propagator and thus no wave function renormalization [15, 17, 56] and only the reaction rate $\alpha$ needs to be renormalized. This can be done by summing up the corresponding diagrams to all orders, with the result that the one-loop beta function is exact. From the renormalization group equations it then follows that the upper critical dimension is at $d^{*}=2$ and that the exponent $y$ describing the decay of the mean particle number (1.1)

\footnotetext{
${ }^{12}$ In distinction to the quantum Hamiltonians written down above, one usually considers 'bosonic' operators, where the number of particles per site is unrestricted. While that does not appear to be an essential feature when restricting attention to purely destructive reactions, this may be different when considering processes which create new particles, see 57, 58.
} 
is given by $y=\min (1, d / 2)$, in agreement with previous heuristic arguments [6, 59]. In particular, it transpires that the whole probability distribution for fluctuations becomes universal in the late time regime 13 .

What is the analogue of the similarity transformation (2.19) in the Lagrangian formulation? To see this, consider the process with only diffusion, annihilation and coagulation present [52]. The action takes the form, where $V$ describes the interactions of the particles undergoing a reaction

$$
S_{\lambda}=\int d t d x \bar{a}\left(\dot{a}-D \nabla^{2} a\right)+\int d t d x d x^{\prime} V\left(\left|x-x^{\prime}\right|\right)\left[\bar{a}(x) \bar{a}\left(x^{\prime}\right)+\lambda \bar{a}(x)\right] a(x) a\left(x^{\prime}\right)
$$

such that for $\lambda=1$ only coagulation and for $\lambda=2$ only annihilation reactions occur. Following [52], consider the potentials $V_{\alpha, \gamma}$ for pure annihilation and coagulation, respectively, to be of the form $V_{\alpha}(x)=\alpha V(x)$ and $V_{\gamma}(x)=\gamma V(x)$. Then

$$
\lambda=\frac{2 \alpha+\gamma}{\alpha+\gamma}
$$

Thus, through the transformation $a \rightarrow \lambda a, \bar{a} \rightarrow \lambda^{-1} \bar{a}$ the coagulation-annihilation model can be reduced to pure coagulation. For the quantum Hamiltonian, the corresponding transformation matrix is $B=$

$\left(\begin{array}{cc}1 & 1-\lambda \\ 0 & \lambda\end{array}\right)$ 10, 39]. In both formalisms, one arrives at the same relationship between the $n$-point correlators of the two models [10, 39, 52]

$$
\begin{aligned}
& \left\langle n\left(x_{1}, t_{1}\right) \ldots n\left(x_{n}, t_{n}\right)\right\rangle_{\lambda}=\left\langle s\left|a\left(x_{1}, t_{1}\right) \ldots a\left(x_{n}, t_{n}\right)\right| P(t)\right\rangle_{\lambda} \\
= & \lambda^{-n}\left\langle s\left|a\left(x_{1}, t_{1}\right) \ldots a\left(x_{n}, t_{n}\right)\right| P(t)^{\prime}\right\rangle_{1}=\lambda^{-n}\left\langle n\left(x_{1}, t_{1}\right) \ldots n\left(x_{n}, t_{n}\right)\right\rangle_{1}^{\prime}
\end{aligned}
$$

where the primes refer to the rescaling of the initial densities and correlators (since the ave rage of $a$ corresponds to a particle density).

This transformation can be extended to more general systems [60].

\section{Stochastic systems from free fermions}

In this section we give the classification of those stochastic systems which can be obtained from a free fermion Hamiltonian through the transformation (2.19).

\subsection{Calculation of the matrix elements}

Our starting point is the quantum chain

$$
H=-\sum_{j=1}^{L}\left[D_{1} \sigma_{j}^{+} \sigma_{j+1}^{-}+D_{2} \sigma_{j}^{-} \sigma_{j+1}^{+}+\eta_{1} \sigma_{j}^{+} \sigma_{j+1}^{+}+\eta_{2} \sigma_{j}^{-} \sigma_{j+1}^{-}+h_{1} \sigma_{j}^{z}+h_{2} \sigma_{j+1}^{z}-C\right]
$$


which is diagonalizable through a Jordan-Wigner transformation followed by a Bogoliubov transformation or by a canonical transformation as described in appendix A. Here, $D_{1,2}, \eta_{1,2}, h_{1,2}$ are free parameters. In the following, we write $h=h_{1}+h_{2}, g=h_{1}-h_{2}$ and $\eta^{2}=\eta_{1} \eta_{2}$. We shall consider in this section $\eta \neq 0$. The case $\eta=0$ is a special case of a Hamiltonian with $X X Z$ spectrum and will be considered in the next section. Using (2.20), we can write down the matrix describing the nearest-neighbor interactions

$$
H_{j, j+1}=-\left(\begin{array}{cccc}
h-C & 0 & 0 & \eta_{1} \\
0 & g-C & D_{1} & 0 \\
0 & D_{2} & -g-C & 0 \\
\eta_{2} & 0 & 0 & -h-C
\end{array}\right)
$$

and we look for a matrix $\widetilde{H}$ through the transformation

$$
\widetilde{H}=\mathcal{B H B}^{-1}, \mathcal{B}=\bigotimes_{j=1}^{L} B_{j}
$$

and describing a stochastic system. Following the logic described in the preceding section we want to calculate correlation functions of the stochastic system in terms of correlators of the non-stochastic model which we can solve. We therefore rewrite a correlator

$$
\left\langle s\left|\tilde{n}_{x_{1}}\left(t_{1}\right) \ldots \tilde{n}_{x_{k}}\left(t_{k}\right)\right| P_{0}\right\rangle=\left\langle s\left|\tilde{n}_{x_{1}} \exp \left(-\widetilde{H}\left(t_{1}-t_{2}\right)\right) \tilde{n}_{x_{2}} \ldots \tilde{n}_{x_{k}} \exp \left(-\widetilde{H} t_{k}\right)\right| P_{0}\right\rangle
$$

in terms of operators transformed using $B$, thus reducing this correlator to a matrix element computable through free fermion techniques. Inverting this strategy, one may first calculate matrix elements in the free fermion system, and then obtain the corresponding quantity for all stochastic processes related to this system by choosing an appropriate $B$. This is the strength of this approach, illustrated in section 5 for the density $\tilde{\rho}(t)$ as a function of time for an uncorrelated initial state with initial density $\tilde{\rho}_{0}$. A single calculation gives $\tilde{\rho}(t)$ for all stochastic processes related to the free fermion system (3.1).

In order to find a convenient parametrization for $B$ we first fix the determinant of $B$ to be 1 which is no loss of generality since $B$ must be non-singular and $\widetilde{H}$ is independent of $\operatorname{det} B$. We can then write $B$ in a parametrization analogous to the Euler angles (alternatives are discussed in appendix B)

$$
B=\left(\begin{array}{cc}
\sqrt{a} & 0 \\
0 & \sqrt{a^{-1}}
\end{array}\right)\left(\begin{array}{cc}
\cosh \phi & \sinh \phi \\
\sinh \phi & \cosh \phi
\end{array}\right)\left(\begin{array}{cc}
b & 0 \\
0 & b^{-1}
\end{array}\right)
$$

with transformation parameters $a, b, \phi$. Of these, we can always arrange for $b=1$, since otherwise, its effect could be absorbed by redefining $\eta_{1} \rightarrow b^{4} \eta_{1}$ and $\eta_{2} \rightarrow b^{-4} \eta_{2}$. So we are left with just two transformation parameters $a$ and $\phi$. Below, we shall work with $Y$ defined by

$$
Y=e^{2 \phi}
$$


In order to ensure that $\widetilde{H}$ is stochastic we shall have to satisfy the conditions (2.8). Let $\widetilde{C}_{m}=$ $\sum_{k=1}^{4}\left(\widetilde{H}_{j, j+1}\right)_{k, m}$ denote the sum of the elements of the $m$-th column of $\widetilde{H}$. Then, up to boundary terms in $\sigma_{1, L}^{z}$ (which are absent for periodic boundary conditions) and up to an additive constant in $\widetilde{H}$ (which will be fixed later by specifying the constant $C$ ), probability conservation (2.8) is implemented by demanding that

$$
\widetilde{C}_{1}=\widetilde{C}_{4}=\frac{1}{2}\left(\widetilde{C}_{2}+\widetilde{C}_{3}\right)
$$

These are solved by fixing $\eta_{1,2}$

$$
\eta_{1}=\frac{\left(D_{1}+D_{2}+2 h\right)(1-a+Y+a Y)^{2}}{2(-1+a+Y+a Y)^{2}}, \quad \eta_{2}=\frac{\left(D_{1}+D_{2}-2 h\right)(-1+a+Y+a Y)^{2}}{2(1-a+Y+a Y)^{2}}
$$

The vanishing of one of the denominators is not dangerous here, because then either $\eta_{1}$ or $\eta_{2}$ would have to vanish. Systems of this kind are discussed in section 4.

For the discussion of the positivity conditions on the rates it is convenient to introduce the parity symmetric and parity antisymmetric combinations of reaction rates $\varphi_{L, R}$ by

$$
\varphi=\frac{1}{2}\left(\varphi_{L}+\varphi_{R}\right) \quad, \quad \varphi^{\prime}=\frac{1}{2}\left(\varphi_{L}-\varphi_{R}\right)
$$

respectively, where $\varphi$ is taken to be a collective symbol for any member of the set $\{\beta, \gamma, \delta, \sigma, D\}$, see table 1. In order to satisfy the positivity conditions for the stochastic rates appearing in $\widetilde{H}$ one might think that all off-diagonal elements in $\widetilde{H}_{j, j+1}$ have to be negative or zero. The actual conditions on these matrix elements are, however, weaker. To see this, it is useful to write the nearest-neighbor interactions in $\widetilde{H}$ in form of the matrix

$$
\widetilde{H}_{j, j+1}=\left(\begin{array}{cccc}
\widetilde{\sigma}_{L}+\widetilde{\sigma}_{R}+2 \widetilde{\nu} & -\widetilde{\delta}_{R}+y & -\widetilde{\delta}_{L}-y & -2 \widetilde{\alpha} \\
-\widetilde{\sigma}_{R}+x & \left(\widetilde{\delta}_{R}+\widetilde{\beta}_{L}+\widetilde{D}_{L}\right)+x-y & -\widetilde{D}_{R} & -\widetilde{\gamma}_{R}-y \\
-\widetilde{\sigma}_{L}-x & -\widetilde{D}_{L} & \left(\widetilde{\delta}_{L}+\widetilde{\beta}_{R}+\widetilde{D}_{R}\right)-x+y & -\widetilde{\gamma}_{L}+y \\
-2 \widetilde{\nu} & -\widetilde{\beta}_{L}-x & -\widetilde{\beta}_{R}+x & 2 \widetilde{\alpha}+\widetilde{\gamma}_{R}+\widetilde{\gamma}_{L}
\end{array}\right)
$$

where $x, y$ are free parameters. This matrix form corresponds to the decomposition

$$
\widetilde{H}_{j, j+1}=\widetilde{H}_{j, j+1}^{\mathrm{phys}}+x\left(\sigma_{j+1}^{-}-\sigma_{j}^{-}\right)+y\left(\sigma_{j+1}^{+}-\sigma_{j}^{+}\right)
$$

where $\widetilde{H}_{j, j+1}$ is the transformed matrix which in general is non-stochastic, $\widetilde{H}_{j, j+1}^{\text {phys }}$ contains the physical reaction rates and is stochastic and the last two terms parametrized by $x$ and $y$ are divergence terms. For periodic boundary conditions, these extra terms cancel, while for other boundaries, they correspond to surface fields. 
In this paper, we study exclusively periodic boundary conditions. Thus it is sufficient to impose the positivity conditons (2.8) on the parity symmetric combinations of the matrix elements of $\widetilde{H}$ and on two other combinations of transformed matrix elements in which the terms containing $x, y$ cancel. This reduces the number of inequalities one has to satisfy by two. It is important to keep this distinction between the transformed matrix elements (which occur in $\widetilde{H}_{j, j+1}$ ) and the actual rates of the stochastic system (which occur in $\widetilde{H}_{j, j+1}^{\mathrm{phys}}$ ) which have all to be positive.

Next, we have to write down the elements of the transformed matrix $\widetilde{H}_{j, j+1}$ in terms of the matrix elements of $H_{j, j+1}$ and the transformation parameters. To simplify expressions, we make use of the freedom to take the normalization of time scale

$$
D_{1}+D_{2}=2
$$

and we shall consider the other possible case, when $D_{1}+D_{2}=0$, in appendix B. Using the convention of (3.9), we get for the parity symmetric matrix elements

$$
\begin{aligned}
-2 \widetilde{\alpha} & =a^{2}\left(-1+a-Y^{2}-a Y^{2}\right) \cdot F_{1} \cdot N \\
-\widetilde{\gamma} & =\frac{1}{2} a\left(a^{2}-1\right)\left(Y^{2}-1\right) \cdot F_{1} \cdot N \\
-2 \widetilde{\nu} & =a^{-1}\left(-1+a+Y^{2}+a Y^{2}\right) \cdot F_{2} \cdot N \\
-\widetilde{\sigma} & =\frac{1}{2 a}\left(a^{2}-1\right)\left(Y^{2}-1\right) \cdot F_{2} \cdot N \\
-\widetilde{\delta} & =a\left(Y^{2}-1\right)\left(1-a+Y^{2}+a Y^{2}\right) \cdot F_{3} \cdot N \\
-\widetilde{\beta} & =-\left(Y^{2}-1\right)\left(-1+a+Y^{2}+a Y^{2}\right) \cdot F_{3} \cdot N \\
-\widetilde{D} & =\frac{1}{2} F_{4} \cdot N
\end{aligned}
$$

where

$$
\begin{aligned}
F_{1} & =h-2 a h+a^{2} h+4 Y-4 a Y+6 h Y^{2}-2 a^{2} h Y^{2}+4 Y^{3}+4 a Y^{3}+h Y^{4}+2 a h Y^{4}+a^{2} h Y^{4} \\
F_{2} & =h-2 a h+a^{2} h+4 a Y-4 a^{2} Y-2 h Y^{2}+6 a^{2} h Y^{2}-4 a Y^{3}-4 a^{2} Y^{3}+h Y^{4}+2 a h Y^{4}+a^{2} h Y^{4} \\
F_{3} & =h-2 a h+a^{2} h+2 Y-2 a^{2} Y+h Y^{2}+2 a h Y^{2}+a^{2} h Y^{2} \\
F_{4} & =h-2 a h+2 a^{3} h-a^{4} h+8 a Y-16 a^{2} Y+8 a^{3} Y-h Y^{2}+6 a h Y-6 a^{3} h Y^{2}+a^{4} h Y^{2} \\
& -h Y^{4}-6 a h Y^{4}+6 a^{3} h Y^{4}+a^{4} h Y^{4}-8 a Y^{5}-16 a^{2} Y^{5}-8 a^{3} Y^{5} \\
& +h Y^{6}+2 a h Y^{6}-2 a^{3} h Y^{6}-a^{4} h Y^{6} \\
N^{-1} & =Y(1-a+Y+a Y)^{2}(-1+a+Y+a Y)^{2}
\end{aligned}
$$


It is remarkable that the matrix elements can be factorised in terms of only very few building blocks. This observation is going to be essential in the classification of the possible stochastic systems. Whether this factorization property has a deeper algebraic meaning is still open. Note that if we use the scaling $\frac{1}{2}\left(D_{1}+D_{2}\right)=-1$, we recover the same expressions with $Y \rightarrow-Y$, which gives nothing new. The parity-antisymmetric matrix elements are

$$
\begin{aligned}
\widetilde{\gamma}^{\prime}-y & =\left(D_{1}-D_{2}+2 g\right) \frac{1}{8 Y} a\left(Y^{2}-1\right) \\
\widetilde{\sigma}^{\prime}+x & =\left(D_{1}-D_{2}+2 g\right) \frac{1}{8 a Y}\left(Y^{2}-1\right) \\
\widetilde{\delta}^{\prime}+y & =\left(D_{1}-D_{2}-2 g\right) \frac{1}{8 Y} a\left(Y^{2}-1\right) \\
-\widetilde{\beta}^{\prime}-x & =\left(D_{1}-D_{2}-2 g\right) \frac{1}{8 a Y}\left(Y^{2}-1\right) \\
\widetilde{D^{\prime}} & =-\left(D_{1}-D_{2}\right) \frac{1}{4 Y}\left(Y^{2}+1\right)
\end{aligned}
$$

In order to get a stochastic system from the matrix elements (3.13, 3.15), we must implement the positivity conditions (2.8) on the parity symmetric rates $\widetilde{\alpha}, \widetilde{\gamma}, \widetilde{\nu}, \widetilde{\sigma}, \widetilde{\delta}, \widetilde{\beta}, \widetilde{D}$. These conditions completely determine the transformation matrix $B$. The physical systems thus found will have left-right symmetric reaction-diffusion rates. Then, in a second step and using the extra freedom coming from adding the divergence terms to $\widetilde{H}_{j, j+1}^{\text {phys }}$ as done in (3.11), we shall identify free fermion systems with left-right biased diffusion and reaction rates.

We now turn to the analysis of the positivity conditions (2.8).

\subsection{Reality of the transformation parameters $a, Y$}

Before embarking on checking the positivity of the symmetric rates (3.13), it is useful to break the problem into smaller pieces. It is a necessary condition to positivity that all matrix elements of $\widetilde{H}$ are real. This reality condition allows us to distinguish a few subcases. First, for $Y^{2}=1$, the transformation from $H$ to $\widetilde{H}$ merely amounts to the diagonal transformation encountered before 61]. So from now on we always assume $Y \neq 1,-1$. Second, since the ratios $\widetilde{\gamma}^{\prime} / \widetilde{\sigma}^{\prime}$ and $\widetilde{\delta}^{\prime} / \widetilde{\beta}^{\prime}$ must be real, it follows from (3.15) that

$$
a^{2} \text { real }
$$

and we distinguish the following.

1. a real. Then, since $2 \widetilde{\alpha} / \widetilde{\gamma}$ is real, it follows that

$$
\frac{a\left(-1+a-Y^{2}-a Y^{2}\right)}{\left(a^{2}-1\right)\left(Y^{2}-1\right)} \text { real }
$$


and it follows that $Y^{2}$ must be real. In the first case, $Y$ itself is real, and reality of $\widetilde{H}$ is guaranteed if $h, g$ and $D_{1}-D_{2}$ are real. In the second case, $Y$ is purely imaginary and reality of the elements of $\widetilde{H}$ is assured if $D_{1}-D_{2}, h$ and $g$ are all imaginary.

2. $a$ imaginary. We write $a=i A$ with $A$ real. Since $2 \widetilde{\nu} / \widetilde{\sigma}$ must be real, it follows from (3.13) that

$$
\frac{-1+a+Y^{2}+a Y^{2}}{\left(a^{2}-1\right)\left(Y^{2}-1\right)} \text { real }
$$

which can be rewritten as

$$
\Re \frac{Y^{2}+1}{Y^{2}-1}=0
$$

or, letting $Y^{2}=P+i Q$, we find that $P^{2}+Q^{2}=1$ and thus have

$$
\left|Y^{2}\right|^{2}=1 \text { if } a \text { is imaginary }
$$

In summary, we have to consider the following cases separately

A) $a$ real and $Y$ real, B) $a$ real and $Y$ imaginary , C) $a$ imaginary and $|Y|=1$

and we now ask when the conditions $\widetilde{\alpha}, \widetilde{\beta}, \widetilde{\gamma}, \widetilde{\delta}, \widetilde{\nu}, \widetilde{\sigma}, \widetilde{D} \geq 0$ can be simultaneously met.

\subsection{A no-go theorem}

In this section, we prove the lemma stated below. The reader not interested in the details of the proof can go on to the next section, where the implications will be discussed.

Lemma: For the parity-symmetric reaction rates $\widetilde{\alpha}, \widetilde{\gamma}, \widetilde{\nu}$ and $\widetilde{\sigma}$ from eq. (3.13) with the scaling $\frac{1}{2}\left(D_{1}+\right.$ $\left.D_{2}\right)=1$, the inequalities

$$
\widetilde{\alpha}>0, \widetilde{\gamma}>0, \widetilde{\nu}>0, \tilde{\sigma}>0
$$

cannot be simultaneously satisfied.

Proof: We show this by examining each of the cases in (3.21) separately. For the sake of clarity and brevity, we are not going to go through the inequalities explicitly, but rather prefer to illustrate graphically what is happening. Once having understood from the graphics the result to be proved, it is a straightforward matter to check the relevant inequalities. The matrix elements depend on the three free parameters $a, Y$ and $h$. We are interested in localising the regions where the rates $\widetilde{\varphi}$ (where as in (3.9), $\widetilde{\varphi}$ can stand for any of the rates $\widetilde{\alpha}, \widetilde{\gamma}, \widetilde{\nu}, \widetilde{\sigma})$ have a constant sign. These regions are bounded by those lines along which the $\widetilde{\varphi}$ have a simple zero or a simple pole. We now fix $h$ and look for the curves 
$a=a(Y)$ such that the rates $\widetilde{\varphi}$ vanish. These curves are readily identified from the factorized form of the matrix elements in eq. (3.13).

\section{Case A: $a, Y$ both real.}

For $h$ fixed, the changes of sign of $\widetilde{\alpha}$ are along the curves

$$
\begin{aligned}
a & =\frac{1+Y^{2}}{1-Y^{2}} \\
a_{ \pm} & =\left[h\left(1-Y^{2}\right)\right]^{-1}\left[h\left(1+Y^{2}\right)+2 Y\left(1 \pm \sqrt{1-h^{2}}\right)\right] \\
Y & =0
\end{aligned}
$$

$\widetilde{\gamma}$ changes sign along the curves

$$
\begin{aligned}
a_{ \pm} & =\left[h\left(1-Y^{2}\right)\right]^{-1}\left[h\left(1+Y^{2}\right)+2 Y\left(1 \pm \sqrt{1-h^{2}}\right)\right] \\
a & = \pm 1, \quad a=0 \\
Y & = \pm 1, \quad Y=0
\end{aligned}
$$

$\widetilde{\nu}$ changes sign along the curves

$$
\begin{aligned}
a & =\frac{1-Y^{2}}{1+Y^{2}} \\
a_{ \pm} & =\left[h-4 Y+6 h Y^{2}-4 Y^{3}+h Y^{4}\right]^{-1}\left[\left(1-Y^{2}\right)\left(h\left(1+Y^{2}\right)-2 Y\left(1 \pm \sqrt{1-h^{2}}\right)\right)\right] \\
a & =0 \\
Y & =0
\end{aligned}
$$

$\widetilde{\sigma}$ changes sign along the curves

$$
\begin{aligned}
a_{ \pm} & =\left[h-4 Y+6 h Y^{2}-4 Y^{3}+h Y^{4}\right]^{-1}\left[\left(1-Y^{2}\right)\left(h\left(1+Y^{2}\right)-2 Y\left(1 \pm \sqrt{1-h^{2}}\right)\right)\right] \\
a & = \pm 1, \quad a=0 \\
Y & = \pm 1, \quad Y=0
\end{aligned}
$$

Next, we have to distinguish between the cases $h^{2}>1$ and $h^{2} \leq 1$. In the first case, the $a_{ \pm}$given above are complex and will not play a role in dividing the $(a, Y)$ plane into regions where the $\widetilde{\varphi}$ are positive or negative, respectively. In fact, in this case the boundaries are completely independent of $h$. In figure 1 , we show the excluded (shaded) regions for the four rates $\widetilde{\alpha}, \widetilde{\gamma}, \widetilde{\nu}, \widetilde{\sigma}$. Comparing them, it follows that no region in the $(a, Y)$ plane remains where the four inequalities (3.22) are all satisfied.

In the second case, the $a_{ \pm}$are all real and must be taken into account. In order to illustrate the mutual exclusion of the allowed regions, we show in figure 2 the excluded (shaded) regions for the four 
rates for the special value $h=\frac{1}{2} \sqrt{3}$. In comparing the excluded regions for the different rates, note that because of the factor structure of the matrix elements, the boundary curves in the different figures coincide. Again, it is apparent that (3.22) cannot be satisfied.

Case B: a real and $Y$ imaginary.

We write $Y=i y$ and $h=i k$ where $y, k$ are both real. We fix $k$ and look for the curves in the $(a, y)$ plane where the $\widetilde{\varphi}$ have simple zeroes or poles. For $\widetilde{\alpha}$, these occur at

$$
\begin{aligned}
& a=\frac{1-y^{2}}{1+y^{2}} \\
& a=\left[k\left(1+y^{2}\right)\right]^{-1}\left[k\left(1-y^{2}\right)+2 y\left(1 \pm \sqrt{1+k^{2}}\right)\right] \\
& y=0
\end{aligned}
$$

$\widetilde{\gamma}$ changes sign at

$$
\begin{aligned}
& a=\left[k\left(1+y^{2}\right)\right]^{-1}\left[k\left(1-y^{2}\right)+2 y\left(1 \pm \sqrt{1+k^{2}}\right)\right] \\
& a= \pm 1, \quad a=0 \\
& y=0
\end{aligned}
$$

$\widetilde{\nu}$ changes sign at

$$
\begin{aligned}
& a=\frac{1-y^{2}}{1+y^{2}} \\
& a=\left[k-4 y-6 k y^{2}+4 y^{3}+k y^{4}\right]^{-1}\left[\left(1+y^{2}\right)\left(k\left(1-y^{2}\right)-2 y\left(1 \pm \sqrt{1+k^{2}}\right)\right)\right] \\
& a=0 \\
& y=0
\end{aligned}
$$

$\widetilde{\sigma}$ changes sign at

$$
\begin{aligned}
& a=\left[k-4 y-6 k y^{2}+4 y^{3}+k y^{4}\right]^{-1}\left[\left(1+y^{2}\right)\left(k\left(1-y^{2}\right)-2 y\left(1 \pm \sqrt{1+k^{2}}\right)\right)\right] \\
& a= \pm 1, \quad a=0 \\
& y=0
\end{aligned}
$$

In analogy what was done in case $\mathrm{A}$, one can show that each region in the $(a, y)$ plane is excluded by at least one of the four rates and thus (3.22) cannot be satisfied.

Case C: $a$ imaginary and $|Y|=1$.

We write $a=i A$ and $Y=\cos q+i \sin q$ with $A, q$ real. We fix $h$ and look for the simple zeroes or poles 
of the rates $\widetilde{\varphi}$. For $\widetilde{\alpha}$, these occur at

$$
\begin{aligned}
A_{ \pm} & =h^{-1} \csc q \cdot\left(1 \pm \sqrt{1-h^{2}}+h \cos q\right) \\
A & =\cot q
\end{aligned}
$$

For $\widetilde{\gamma}$, they occur at

$$
\begin{aligned}
A_{ \pm} & =h^{-1} \csc q \cdot\left(1 \pm \sqrt{1-h^{2}}+h \cos q\right) \\
A & =0 \\
q & =0, \pm \pi
\end{aligned}
$$

For $\widetilde{\nu}$, they occur at

$$
\begin{aligned}
A_{ \pm} & =[-3 h+4 \cos q-h \cos 2 q]^{-1}\left[2 \sin q \cdot\left(-1 \pm \sqrt{1-h^{2}}+h \cos q\right)\right] \\
A & =-\tan q \\
A & =0
\end{aligned}
$$

and for $\tilde{\sigma}$, they occur at

$$
\begin{aligned}
A_{ \pm} & =[-3 h+4 \cos q-h \cos 2 q]^{-1}\left[2 \sin q \cdot\left(-1 \pm \sqrt{1-h^{2}}+h \cos q\right)\right] \\
A & =0 \\
q & =0, \pm \pi
\end{aligned}
$$

For $h^{2}>1$, the $A_{ \pm}$are complex and can be ignored. For $h^{2} \leq 1$, the $A_{ \pm}$are real. To illustrate the mutual exclusion of the positivity conditions, we display in figure 3 the graphs for the case $h^{2}>1$. Again, it is apparent that (3.22) cannot be all satisfied. The case $h^{2}<1$ is treated similarly.

This proves the assertion.

q.e.d.

\subsection{The parity-symmetric stochastic systems}

The lemma just proven severely restricts the possibilities for the choice of the transformation parameters

$a, Y$ and still find a stochastic quantum Hamiltonian $\widetilde{H}$. In fact, it follows that at least one of the rates $\widetilde{\alpha}, \widetilde{\gamma}, \widetilde{\nu}, \widetilde{\sigma}$ has to vanish. But because of the explicit factorization (3.13) of the matrix elements, this means that two of the four rates considered will be zero. Comparing with (3.13), we see that one of the following conditions must be implemented

$$
F_{1}=0, \quad F_{2}=0,-1+a-Y^{2}-a Y^{2}=0,-1+a+Y^{2}+a Y^{2}=0, a^{2}=1
$$


We turn to them one by one.

1. $F_{1}=0$. This means that $\widetilde{\alpha}=\widetilde{\gamma}=0$. We solve this for $h$ and get

$$
\begin{aligned}
h & =-4 Y\left(1-a+Y^{2}+a Y^{2}\right) N_{1} \\
N_{1}^{-1} & =1-2 a+a^{2}+6 Y^{2}-2 a^{2} Y^{2}+Y^{4}+2 a Y^{4}+a^{2} Y^{4}
\end{aligned}
$$

and the non-vanishing rates then become

$$
\begin{aligned}
-2 \widetilde{\nu} & =\frac{4}{a}\left(1+Y^{2}\right)\left(1-a-Y^{2}-a Y^{2}\right) N_{1} \\
-\widetilde{\delta} & =2 a\left(1-Y^{2}\right)\left(1-a+Y^{2}+a Y^{2}\right) N_{1} \\
-\widetilde{\beta} & =2\left(Y^{2}-1\right)\left(-1+a+Y^{2}+a Y^{2}\right) N_{1} \\
-\widetilde{\sigma} & =\frac{2}{a}\left(a^{2}-1\right)\left(1-Y^{2}\right)\left(1+Y^{2}\right) N_{1} \\
-\widetilde{D} & =2\left(1+Y^{2}\right)\left(-1+a-Y^{2}-a Y^{2}\right) N_{1}
\end{aligned}
$$

A necessary condition for positivity are the relations

$$
\frac{2 \widetilde{\nu}}{\widetilde{\beta}} \geq 0, \frac{\widetilde{D}}{\widetilde{\delta}} \geq 0
$$

Provided none of the factors in the rates vanishes, we then find

$$
0 \leq \frac{1}{a} \frac{Y^{2}+1}{Y^{2}-1} \leq 0
$$

This means that one of the following conditions must be satisfied:

$$
\text { i) } Y= \pm \sqrt{\frac{1-a}{1+a}}, \text { ii) } Y= \pm \sqrt{\frac{a-1}{a+1}}, \text { iii) } Y= \pm i
$$

In the first case, we write $a=i A$ with $A$ real, i.e. $Y^{2}=(1-i A) /(1+i A)$, and find that

$$
\widetilde{H}_{j, j+1}=\frac{1}{2+A^{2}}\left(\begin{array}{cccc}
4 & -2 A^{2} & -2 A^{2} & 0 \\
-2 & 2+2 A^{2} & -2 & 0 \\
-2 & -2 & 2+2 A^{2} & 0 \\
0 & 0 & 0 & 0
\end{array}\right)
$$

does correspond to a stochastic system, which we call model I. We identify

$$
C=1 \quad, \quad h=-2 \frac{\sqrt{1+A^{2}}}{2+A^{2}} \quad, \quad \eta_{1}=\eta_{2}=-\frac{A^{2}}{2+A^{2}} \quad, \quad D_{1}=D_{2}=1
$$

In the second case, we get

$$
\widetilde{\nu}=\widetilde{\beta}=-\widetilde{\sigma}=2
$$


which is not stochastic. Finally, the third case $Y^{2}=-1$ gives, with $a=i A$ and $A$ real

$$
\widetilde{H}_{j, j+1}=\frac{1}{1+A^{2}}\left(\begin{array}{cccc}
0 & -2 A^{2} & -2 A^{2} & 0 \\
0 & 2+2 A^{2} & 0 & 0 \\
0 & 0 & 2+2 A^{2} & 0 \\
0 & -2 & -2 & 0
\end{array}\right)
$$

which is again stochastic, is called model III, and we identify

$$
C=1, \quad h=\frac{2 A}{1+A^{2}}, \quad \eta_{1}=-\frac{(A-1)^{2}}{1+A^{2}}, \quad \eta_{2}=-\frac{(A+1)^{2}}{1+A^{2}}, \quad D_{1}=D_{2}=1
$$

2. $F_{2}=0$. This means that $\widetilde{\nu}=\widetilde{\sigma}=0$. Solving for $h$ we find

$$
\begin{aligned}
h & =4 a Y\left(-1+a+Y^{2}+a Y^{2}\right) N_{2} \\
N_{2}^{-1} & =1-2 a+a^{2}-2 Y^{2}+6 a^{2} Y^{2}+Y^{4}+2 a Y^{4}+a^{2} Y^{4}
\end{aligned}
$$

The non-vanishing rates are now found as above and the discussion is completely parallel. We take $Y=\sqrt{(a-1) /(a+1)}$ and $a=i A^{-1}$ and get the stochastic quantum Hamiltonian

$$
\widetilde{H}_{j, j+1}=\frac{1}{2+A^{2}}\left(\begin{array}{cccc}
0 & 0 & 0 & 0 \\
0 & 2+2 A^{2} & -2 & -2 \\
0 & -2 & 2+2 A^{2} & -2 \\
0 & -2 A^{2} & -2 A^{2} & 4
\end{array}\right)
$$

which is called model II and we identify

$$
C=1, \quad h=2 \frac{\sqrt{1+A^{2}}}{2+A^{2}}, \quad \eta_{1}=\eta_{2}=-\frac{A^{2}}{2+A^{2}}, \quad D_{1}=D_{2}=1
$$

This transformation of model II to free fermions had been given before [38. Note that the only difference with model I (3.41) occurs in the sign of $h$, which expresses the fact that the two models I and II are related by a particle-hole exchange. The other possibilities (analogous to (3.40)) do not give anything new.

3. $Y= \pm \sqrt{(a-1) /(a+1)}$. We write again $a=i A^{-1}$ and find in particular

$$
-\widetilde{\sigma}=4 \widetilde{\nu}=2-h \frac{2+A^{2}}{\sqrt{1+A^{2}}}
$$

This can only be made stochastic if $\widetilde{\nu}=\widetilde{\sigma}=0$. But then $h=2 \sqrt{1+A^{2}} /\left(2+A^{2}\right)$ and we are back to the case (3.47) already found.

4. $Y= \pm \sqrt{(1-a) /(1+a)}$. In the same way, we find that $h=-2 \sqrt{1+A^{2}} /\left(2+A^{2}\right)$ and we recover the system (3.41). 
5. $a^{2}=1$. We find that $\tilde{\delta} / \widetilde{\beta}=-1$. Now $\widetilde{\delta}=h\left(1-Y^{2}\right) /(2 Y)$ which only vanishes for $h=0$ or $Y^{2}=1$. In both cases, the transformation $\mathcal{B}$ is diagonal and we are back to the systems studied in [61].

In conclusion, we have found the complete list of parity-symmetric stochastic systems which can be reduced to a free fermion model with site-independent interactions through the transformation (2.19). These are given in (3.47), (3.41), (3.44) and describe particles undergoing coagulation/decoagulation with the coagulation rate equal to the diffusion rate (in this case, the transformation was already known [38]), the model obtained from the latter one when particles and holes are exchanged and finally the so-called biased voter model, see [62]. This correspondence is not immediately obvious and is explained in appendix $\mathrm{C}$.

\subsection{Stochastic systems with a drift}

Having found the set of transformations which render the symmetric rates $\widetilde{\varphi}$ positive, we can now extend the identification with free fermion systems to models including a drift.

We illustrate the procedure for the case when $F_{1}=0$ and then proceed to list the results. When $F_{1}=0$, we have $\widetilde{\gamma}=0$ and thus $\widetilde{\gamma}_{L}=\widetilde{\gamma}_{R}=0$, since both must be positive. From (3.15), this fixes $y=\left(D_{1}-D_{2}+2 g\right) a\left(1-Y^{2}\right) /(8 Y)$. But on the other hand, we have

$$
\begin{aligned}
\widetilde{\delta}_{L}+\widetilde{\delta}_{R} & =2 \widetilde{\delta} \\
\widetilde{\delta}_{L}-\widetilde{\delta}_{R}+2 y & =\left(D_{1}-D_{2}-2 g\right) \frac{a\left(Y^{2}-1\right)}{4 Y}
\end{aligned}
$$

Thus, solving for $\widetilde{\delta}_{L, R}$, we find

$$
\widetilde{\delta}_{L, R}=\widetilde{\delta} \pm \frac{a\left(Y^{2}-1\right)}{4 Y}\left(D_{1}-D_{2}\right)
$$

Similar considerations apply for the other rates. We can now list the stochastic systems with a drift which can be mapped to a free fermion system via (2.19).

1. Model I with death/creation process (3.41) extends to the following system with the only nonvanishing rates

$$
\widetilde{\delta}_{R, L}=A^{2} \widetilde{\sigma}_{L, R}=A^{2} \widetilde{D}_{L, R}=\frac{2 A^{2}}{2+A^{2}} \mp \frac{A^{2}}{2 \sqrt{1+A^{2}}}\left(D_{1}-D_{2}\right)
$$

and the surface fields are given by

$$
x=\frac{1}{4}\left(D_{1}-D_{2}-2 g\right) \frac{1}{\sqrt{A^{2}+1}}, y=-\frac{1}{4}\left(D_{1}-D_{2}+2 g\right) \frac{A^{2}}{\sqrt{A^{2}+1}}
$$

The positivity of the rates restricts $D_{1}-D_{2}$ to a certain interval whose boundaries depend only on the real parameter $A$. 
2. For the coagulation/decoagulation model II (3.47) we get the following system with the nonvanishing rates

$$
\widetilde{\beta}_{L, R}=A^{2} \widetilde{\gamma}_{L, R}=A^{2} \widetilde{D}_{L, R}=\frac{2 A^{2}}{2+A^{2}} \pm \frac{A^{2}}{2 \sqrt{1+A^{2}}}\left(D_{1}-D_{2}\right)
$$

and the surface fields are given by

$$
x=-\frac{1}{4}\left(D_{1}-D_{2}+2 g\right) \frac{A^{2}}{\sqrt{A^{2}+1}}, y=\frac{1}{4}\left(D_{1}-D_{2}-2 g\right) \frac{1}{\sqrt{A^{2}+1}}
$$

For this model, the transformation to free fermions is already known [63].

3. Finally, the biased voter model III (3.44) is extended to the following system with the nonvanishing rates

$$
\widetilde{\delta}_{L, R}=A^{2} \widetilde{\beta}_{L, R}=\frac{2 A^{2}}{1+A^{2}} \pm \frac{A}{2}\left(D_{1}-D_{2}\right)
$$

and the surface fields are given by

$$
x=-\frac{1}{4}\left(D_{1}-D_{2}+2 g\right) A^{-1}, y=-\frac{1}{4}\left(D_{1}-D_{2}+2 g\right) A
$$

In order to calculate the particle number correlators using the free fermion representation, we have to rewrite the particle number operator $\widetilde{n}_{j}(2.9)$ in the free fermion basis. This is straightforward from the known transformations, and thus completely determined from the parity symmetric case. First, for the system I (3.41), we can rewrite the parameters in the transformation matrix (3.5) in the form

$$
A=-\tan (2 \psi), \phi=i \psi, a=i A, \quad b=1
$$

and we want $n_{j}=B^{-1} \widetilde{n}_{j} B$ with $\widetilde{n}_{j}=\left(\begin{array}{ll}0 & 0 \\ 0 & 1\end{array}\right)_{j}$. We find

$$
\begin{aligned}
n_{j} & =\frac{1}{2}\left(\mathbf{1}-\cos 2 \psi \sigma_{j}^{z}+\sin 2 \psi \sigma_{j}^{y}\right) \\
& =\frac{1}{2}\left(\mathbf{1}-\frac{1}{\sqrt{1+A^{2}}} \sigma_{j}^{z}-\frac{A}{\sqrt{1+A^{2}}} \sigma_{j}^{y}\right)
\end{aligned}
$$

Second, for the system III (3.44), the transformation parameters are

$$
\phi=\frac{i \pi}{4}, a=i A, \quad b=1
$$

and we find

$$
n_{j}=\frac{1}{2}\left(\mathbf{1}+\sigma_{j}^{y}\right)
$$


Finally, for the system II (3.47), the transformation parameters are

$$
A=\tan (2 \psi), \phi=i \psi, a=i A^{-1}, b=1
$$

and we have

$$
n_{j}=\frac{1}{2}\left(1-\frac{1}{\sqrt{1+A^{2}}} \sigma_{j}^{z}+\frac{A}{\sqrt{1+A^{2}}} \sigma_{j}^{y}\right)
$$

If one wants to calculate time correlators of the number operators in the stochastic system then one needs the above equalities, the state $\langle s| \mathcal{B}$ and has also to compute the action of $\mathcal{B}^{-1}$ on the initial state. The calculation of $\langle s| \mathcal{B}$ reduces to a single site problem since $\langle s|$ is a factorized state, $\langle s|=(1,1)^{\otimes L}$ and also $\mathcal{B}=B^{\otimes L}$ factorizes. Later on we shall need the identity

$$
(1,1) B=\left(\sqrt{a} b \cosh \phi+\sqrt{a^{-1}} b \sinh \phi, \sqrt{a} b^{-1} \sinh \phi+\sqrt{a^{-1}} b^{-1} \cosh \phi\right)
$$

From this one can, in particular, calculate density correlators for all four cases (see section 5).

\subsection{Summary}

To summarize our classification of the stochastic systems with site-independent interactions which may be transformed through a single-site transformation (3.3) into a free fermion system with siteindependent couplings, we list in table 3 the stochastic systems, as characterized by the non-vanishing reaction rates as defined in table 1, which can be transformed into the free fermion Hamiltonian

$$
H=-\sum_{j}\left[D_{1} \sigma_{j}^{-} \sigma_{j+1}^{+}+D_{2} \sigma_{j}^{+} \sigma_{j+1}^{-}+\eta\left(\sigma_{j}^{-} \sigma_{j+1}^{-}+\sigma_{j}^{+} \sigma_{j+1}^{+}\right)+h_{1} \sigma_{j}^{z}+h_{2} \sigma_{j+1}^{z}-1\right]
$$

with $h=h_{1}+h_{2}$ and $g=h_{1}-h_{2}$. Note that we also used the transformation parameter $b$ in (3.5) and chosen to be $b^{8}=\eta_{1} / \eta_{2}$ to enforce $\eta_{1}=\eta_{2}=\eta$ in table 3. In table 3, we also list the values $x, y$ for the surface fields which are needed to guarantee the stochastic interpretation in case of a drift, when $D_{1} \neq D_{2}$, and the particle number operator (2.9) when transformed into the free fermion basis.

Model I describes particles undergoing creation and diffusion with the same rate and death with an arbitrary rate, with and without a parity-breaking bias. Model II is obtained from this by a particlevacancy exchange and describes particles undergoing coagulation and diffusion with the same rate and decoagulation with an arbitrary rate, with and without a drift. The transformation of this model to free fermions had been given before [38, 63]. Model III is the biased voter model [62] with and without spatial asymmetry. While the voter model is usually defined in terms of particle reactions involving three neighboring sites, we remind the reader in appendix $\mathrm{C}$ how to reformulate it in terms of two-sites 


\begin{tabular}{|c|c|c|c|c|c|}
\hline model & rates / number operator & $x$ & $y$ & $h$ & $\eta$ \\
\hline $\mathrm{I}$ & $\begin{array}{c}\widetilde{\delta}_{R, L}=A^{2} \widetilde{\sigma}_{L, R}=A^{2} \widetilde{D}_{L, R}= \\
A^{2}\left[2 /\left(A^{2}+2\right) \mp\left(D_{1}-D_{2}\right) /\left(2 \sqrt{A^{2}+1}\right)\right] \\
2 n=1-\frac{1}{\sqrt{1+A^{2}}} \sigma^{z}-\frac{A}{\sqrt{1+A^{2}}} \sigma^{y}\end{array}$ & $-\mathcal{D}_{-} \frac{1}{\sqrt{A^{2}+1}}$ & $-\mathcal{D}_{+} \frac{A^{2}}{\sqrt{A^{2}+1}}$ & $-\frac{2 \sqrt{A^{2}+1}}{A^{2}+2}$ & $-\frac{A^{2}}{A^{2}+2}$ \\
\hline II & $\begin{array}{c}\widetilde{\beta}_{L, R}=A^{2} \widetilde{\gamma}_{L, R}=A^{2} \widetilde{D}_{L, R}= \\
A^{2}\left[2 /\left(A^{2}+2\right) \pm\left(D_{1}-D_{2}\right) /\left(2 \sqrt{A^{2}+1}\right)\right] \\
2 n=1-\frac{1}{\sqrt{1+A^{2}}} \sigma^{z}+\frac{A}{\sqrt{1+A^{2}}} \sigma^{y}\end{array}$ & $-\mathcal{D}_{+} \frac{A^{2}}{\sqrt{A^{2}+1}}$ & $-\mathcal{D}_{-} \frac{1}{\sqrt{A^{2}+1}}$ & $\frac{2 \sqrt{A^{2}+1}}{A^{2}+2}$ & $-\frac{A^{2}}{A^{2}+2}$ \\
\hline III & $\begin{array}{c}\widetilde{\delta}_{L, R}=A^{2} \widetilde{\beta}_{L, R}= \\
A^{2}\left[2 /\left(A^{2}+1\right) \pm\left(D_{1}-D_{2}\right) /(2 A)\right] \\
2 n=1-\frac{i}{\sqrt{A^{2}-1}} \sigma^{x}+\frac{A}{\sqrt{A^{2}-1}} \sigma^{y}\end{array}$ & $-\mathcal{D}_{+} / A$ & $-\mathcal{D}_{+} A$ & $\frac{2 A}{A^{2}+1}$ & $\frac{A^{2}-1}{A^{2}+1}$ \\
\hline IV & $\begin{array}{c}\widetilde{\alpha}, \widetilde{\nu}, \widetilde{D}_{L}, \widetilde{D}_{R} \\
2 \widetilde{\alpha}+2 \widetilde{\nu}=\widetilde{D}_{L}+\widetilde{D}_{R}=2,2 g=D_{2}-D_{1} \\
2 n=1-\sigma^{z}\end{array}$ & 0 & 0 & $\widetilde{\alpha}-\widetilde{\nu}$ & $2 \sqrt{\widetilde{\alpha} \widetilde{\nu}}$ \\
\hline
\end{tabular}

Table 3: Stochastic models which can be transformed into free fermions, with the normalization $\left(D_{1}+\right.$ $\left.D_{2}\right) / 2=1$. The rates given are the only non-vanishing ones. $x$ and $y$ are the surface fields and $h$ and $\eta$ the parameters of the free fermion model. $A$ is a free real parameter and the short-hand $\mathcal{D}_{ \pm}= \pm\left(D_{1}-D_{2} \pm 2 g\right) / 4$ is used. We also write the expression for the particle number operator $\tilde{n}$ transformed into the free fermion basis $n=B^{-1} \widetilde{n} B$ and expressed in terms of Pauli matrices such that $\eta_{1}=\eta_{2}=\eta$ in $H$, see eq. (3.65). 
reactions. Finally, model IV describes annihilation and birth together with diffusion with or without a drift and considered in detail in [55, 61]. This last model is trivial from our transformation point of view, because by fixing $h$ and $g$ the original Hamiltonian $H$ is already a stochastic matrix itself and the transformation matrix $B$ will be diagonal.

As expected, the four models share the property that the parameters $h, \eta$ in (3.65) satisfy $h^{2}+\eta^{2}=1$, which is the well-known stochastic line in the free fermion system (3.65). These systems can therefore be mapped via a duality transformation [64] onto the one-dimensional kinetic Ising model with Glauber dynamics. However, since the transformation to free fermions of model IV is trivial and of models I,II and III is not, the correlators to be discussed in the free fermion language to get the particle number correlations are quite different.

Another distinction becomes apparent in the presence of a drift. For models I,II, the divergence terms parametrized by $x$ and $y$ are automatically generated through the similarity transformation. Although for periodic boundary conditions these terms cancel, for non-periodic boundary conditions, they will generate surface fields. In order to find a stochastic system, it will be necessary to add into (3.65) further surface fields and see how they will transform under (2.19). As a consequence, boundary conditions will generically affect the spectrum in an important way. This is different for model III. Here, for a fixed value of the parameter $A$, the couplings $x, y$ of the divergence terms are proportional to each other and the parameter $g$ can be chosen in (3.65) such that $x=y=0$ simultaneously. Then even for non-periodic boundary conditions with a drift, the free fermion Hamiltonian only contains terms which are bilinear in the fermion operators and the techniques of appendix A for its diagonalization can be applied immediately. For model IV the couplings $x$ and $y$ always vanish.

It is of interest to compare the list of free fermion systems of table 3 with the systems obtained through the approach of Peschel et al. [48]. They consider a diffusive coagulation/decoagulation system where in addition birth and creation processes may also be present and identify certain classes of observables for which closed systems of equations of motion can be established and solved. The exitation spectrum of the corresponding quantum Hamiltonian is the one of a XY quantum chain in a site-dependent transverse field [48]. Obviously, our models I,II are special cases of the systems studied in 48], but the voter model is not included there. It remains a challenge to extend the present transformation technique to integrable Hamiltonians with site-dependent interactions and investigate which stochastic systems beyond those studied in [48] exist (in particular, in what directions model III can be extended). 
Finally, the transformation (2.19) we studied is completely local. Although we derived everything here for one-dimensional systems with translational invariance, the transformations as they stand can also be applied to models which are either higher dimensional or contain non-translational invariant interactions, as they might arise for disordered or quasiperiodically modulated systems. Since this type of problem has been much studied for free fermion equilibrium models, see [65] for a review, the techniques developed for equilibrium systems can be carried over to non-equilibrium problems as well.

\section{Stochastic systems from Hamiltonian with $X X Z$ spectrum}

In this section we give the classification of those stochastic systems which can be obtained through the transformation (2.19) from the most general real Hamiltonian having the spectrum of an integrable generalized Heisenberg $X X Z$ quantum chain.

\subsection{Determination of the transformation matrix}

Our starting point is a matrix $H=\sum_{j} H_{j, j+1}$ where $H_{j, j+1}$ is the $4 \times 4$ matrix describing the nearestneighbor interactions ${ }^{13}$

$$
H_{j, j+1}=\left(\begin{array}{cccc}
h_{11} & h_{12} & h_{13} & h_{14} \\
0 & h_{22} & h_{23} & h_{24} \\
0 & h_{32} & h_{33} & h_{34} \\
0 & 0 & 0 & h_{44}
\end{array}\right)
$$

and we look for a matrix $\widetilde{H}$ through the transformation

$$
\widetilde{H}=\mathcal{B H B}^{-1} \quad, \quad \mathcal{B}=\bigotimes_{j=1}^{L} B_{j}
$$

with

$$
B=\left(\begin{array}{ll}
b_{11} & b_{12} \\
b_{21} & b_{22}
\end{array}\right)
$$

and describing a stochastic system.

As pointed out in [24] for a matrix of the form (4.1) the spectrum does not depend on the parameters $h_{12}, h_{13}, h_{14}, h_{24}, h_{34}$. Without these matrix elements, this Hamiltonian can be diagonalized using standard coordinate Bethe ansatz [66, 53]. The eigenvalues of the system with $L$ sites and periodic boundary conditions are

$$
E_{0}=h_{11} L
$$

\footnotetext{
${ }^{13}$ One might also consider a lower triangular form for $H$, which physically merely amounts to a particle-hole interchange.
} 


$$
\begin{aligned}
E\left(p_{1}, \ldots, p_{N}\right)=E_{0} \quad- & \sum_{\ell=1}^{N}\left[\left(2 h_{11}-h_{22}-h_{33}\right)-\left(h_{23}+h_{32}\right) \cos p_{\ell}+\mathrm{i}\left(h_{23}-h_{32}\right) \sin p_{\ell}\right] \\
& 1 \leq N \leq L / 2 \\
E\left(p_{1}, \ldots, p_{N}\right)=E_{L} \quad- & \sum_{\ell=1}^{N}\left[\left(2 h_{44}-h_{22}-h_{33}\right)-\left(h_{23}+h_{32}\right) \cos p_{\ell}-\mathrm{i}\left(h_{23}-h_{32}\right) \sin p_{\ell}\right] \\
& L / 2 \leq N \leq L-1 \\
E_{L}= & h_{44} L
\end{aligned}
$$

where for fixed $N$ the $p_{\ell}$ are given by the Bethe ansatz equations [35]

$$
p_{l}=\frac{2 \pi n_{\ell}+\sum_{p_{j} \neq p_{\ell}} \psi_{p_{\ell} p_{j}}}{L}
$$

where the $n_{\ell}, n_{j}$ are distinct integers and the phase shifts $\psi_{p_{\ell} p_{j}}$ are given by

$$
\cot \left(\frac{\psi_{p_{\ell} p_{j}}}{2}\right)=\frac{\left(h_{11}+h_{44}-h_{22}-h_{33}\right) \sin \left(\frac{p_{\ell}-p_{j}}{2}\right)}{\left(h_{23}+h_{32}\right) \cos \left(\frac{p_{\ell}+p_{j}}{2}\right)-\mathrm{i}\left(h_{23}-h_{32}\right) \sin \left(\frac{p_{\ell}+p_{j}}{2}\right)-\left(h_{11}+h_{44}-h_{22}-h_{33}\right) \cos \left(\frac{p_{\ell}-p_{j}}{2}\right)}
$$

if $1 \leq N \leq L / 2$ and

$$
\cot \left(\frac{\psi_{p_{\ell} p_{j}}}{2}\right)=\frac{\left(h_{11}+h_{44}-h_{22}-h_{33}\right) \sin \left(\frac{p_{\ell}-p_{j}}{2}\right)}{\left(h_{23}+h_{32}\right) \cos \left(\frac{p_{\ell}+p_{j}}{2}\right)+\mathrm{i}\left(h_{23}-h_{32}\right) \sin \left(\frac{p_{\ell}+p_{j}}{2}\right)-\left(h_{11}+h_{44}-h_{22}-h_{33}\right) \cos \left(\frac{p_{\ell}-p_{j}}{2}\right)}
$$

if $L / 2 \leq N \leq L-1$. From these equations we can decide how many steady states the systems has, i.e. how many eigenvalues 0 , and whether the low-lying excitation spectrum has a non-vanishing gap or is gapless.

The first step towards a classification of stochastic processes with a spectrum given by (4.4) - (4.7) consists in determining the matrix $B$. Since $\widetilde{H}$ is stochastic, $\widetilde{H}_{j, j+1}$ has the left ground state

$$
\langle s|=(1,1)^{\otimes^{2}}
$$

which is a product state. This implies that also $H$ will have a left ground state as a product state and related to $\langle s|$

$$
\langle s| B=\langle\omega|=\left(\omega_{1}, \omega_{2}\right)^{\otimes^{2}}=\left(\omega_{1}^{2}, \omega_{1} \omega_{2}, \omega_{1} \omega_{2}, \omega_{2}^{2}\right)
$$

where $\omega_{1,2}$ are free parameter. It follows that we must have

$$
\langle\omega| H_{j, j+1}=\langle\omega|\left(A_{j}-A_{j+1}\right)=(0, x,-x, 0)
$$

where $A_{j}$ is an arbitrary matrix acting on site $j$ and $x$ is an arbitrary constant (depending on $A$ ). Next, we observe from (4.13) that we can always arrange to have $\omega_{1}+\omega_{2}=1$. It is thus enough to keep only $\omega:=\omega_{1}$ as free parameter. 
Now the implementation of eq. (4.13) allows us to distinguish three cases.

Case I: $\omega=0$. In this case $\langle\omega|=(0,0,0,1)$ and by imposing (4.13) we have:

$$
h_{44}=0
$$

whereas, from (4.12):

$$
B=\left(\begin{array}{cc}
b_{11} & 1-b_{22} \\
-b_{11} & b_{22}
\end{array}\right)
$$

Moreover, from the positivity of the spectrum we do have the following inequalities:

$$
\begin{aligned}
h_{11} & \geq 0 \\
\left(h_{23}+h_{32}\right) \cos k+h_{22}+h_{33} & \geq 0 \quad \forall k \in[0,2 \pi]
\end{aligned}
$$

where we have used (4.6) and (4.10) for $N=1$.

Case II: $\omega=1$. The left eigenvector is $\langle\omega|=(1,0,0,0)$ and eq. (4.13) gives:

$$
\begin{aligned}
h_{11} & =0 \\
h_{12}+h_{13} & =0 \\
h_{14} & =0
\end{aligned}
$$

By using eq. (4.12) the transformation matrix $B$ simplifies to:

$$
B=\left(\begin{array}{cc}
b_{11} & -b_{22} \\
1-b_{11} & b_{22}
\end{array}\right)
$$

Positivity of the spectrum translates, in the same way as above, in this case into the following inequalities:

$$
\begin{aligned}
h_{44} & \geq 0 \\
\left(h_{23}+h_{32}\right) \cos k+h_{22}+h_{33} & \geq 0 \quad \forall k \in[0,2 \pi]
\end{aligned}
$$

where we have used (4.6) and (4.10) for $N=L-1$.

Further information can be gained by considering how many steady states exist. If there is a single steady state, we must have

$$
0 \leq b_{11} \leq 1
$$


The important inequality (4.20) is seen as follows. If $h_{11}=0$, the right ground state of $H_{j, j+1}$ is $|0\rangle=(1,0,0,0)=(1,0)^{\otimes^{2}}$. This corresponds to the right ground state $|\widetilde{0}\rangle$ of $\widetilde{H}_{j, j+1}$ through

$$
|\widetilde{0}\rangle=(\widetilde{\rho}, 1-\widetilde{\rho})^{\otimes^{2}}=B_{1} B_{2}|0\rangle
$$

where $\widetilde{\rho}$ is the mean particle density per site in the steady state and obviously $0 \leq \widetilde{\rho} \leq 1$. From the explicit form of the matrix $B$, one has directly

$$
\widetilde{\rho}=b_{11}
$$

and (4.20) follows. In addition, eq. (4.22) allows to calculate $\widetilde{\rho}$ once the transformation matrix is found.

If there are two steady states, one of the following conditions must be satisfied:

$$
\left(h_{23}+h_{32}\right) \cos k+h_{22}+h_{33}=0 \quad \text { for } \quad k=0, \pi
$$

or

$$
h_{44}=0
$$

In these cases, which we shall not treat explicitly, we did not find a similar constraint on the transformation parameter.

Case III: $\omega \neq 0,1$. Since $(\omega, 1-\omega)=(1,1) \mathcal{R}$ with

$$
\mathcal{R}=\left(\begin{array}{cc}
\omega & 0 \\
0 & 1-\omega
\end{array}\right)
$$

condition 4.13 can be written as

$$
\langle\omega| H_{j, j+1}=\langle s| \mathcal{R} \otimes \mathcal{R} H_{j, j+1} \mathcal{R}^{-1} \otimes \mathcal{R}^{-1}=(0, x,-x, 0)
$$

In this way we have "absorbed" the parameter $\omega$ in the coefficients of the Hamiltonian and condition (4.13) simplifies to:

$$
(1,1,1,1) H_{j, j+1}=(0, x,-x, 0)
$$

From eq. (4.26) we then obtain:

$$
\begin{aligned}
h_{11} & =0 \\
h_{12}+h_{13}+h_{22}+h_{33}+h_{23}+h_{32} & =0 \\
h_{14}+h_{24}+h_{34}+h_{44} & =0
\end{aligned}
$$




\begin{tabular}{|c|c|}
\hline parameter & definition \\
\hline$\Delta$ & $\left(h_{23}+h_{32}\right) / P$ \\
\hline$\Omega$ & $h_{24}+h_{34}$ \\
\hline$Q$ & $h_{22}+h_{33}+h_{23}+h_{32}$ \\
\hline$P$ & $h_{44}-Q$ \\
\hline$R$ & $Q /(2 P)$ \\
\hline & Case III \\
\hline$S$ & $\Omega / P \quad 2+(Q+\Omega) / P$ \\
\hline
\end{tabular}

Table 4: Combinations of the coefficients $h_{i j}$ that occur frequently in the text. With the exception of $S$, which is different for the cases II and III, all parameters are defined in the same way in all three cases.

For $B$, eq. (4.12) becomes $(1,1) B=(1,1)$ and we obtain

$$
B=\left(\begin{array}{cc}
b_{11} & 1-b_{22} \\
1-b_{11} & b_{22}
\end{array}\right)
$$

with $\operatorname{det} B=b_{11}+b_{22}-1$. Positivity constraints are the same as in case II. Again, we can distinguish between subcases in which we have one steady steady state or two steady states obtaining the same additional constraints on the parameters that we obtained for case II.

A final comment is in order. From eqs. (4.26) it is clear that $H$ by itself also satisfies the probability conservation condition eq. (2.8) for a stochastic system. Furthermore, the transformation (4.29) has exactly the same form as previously derived [10, 39] for the stochastic similarity transformation between two stochastic systems. Thus, taking the off-diagonal elements of $H$ to be negative, we shall obtain the complete list of two-states stochastic systems which can be reduced through a stochastic similiarity transformation to the integrable model which just contains the rates $\alpha, \gamma$ and $\delta$, corresponding to annihilation, coagulation and death processes, respectively.

By giving the explicit forms for the transformation matrices, we have implemented the probability conservation condition (2.8). It remains to implement the positivity conditions. As we had done before in the case of the free fermion integrable Hamiltonian $H$, we consider first the parity-symmetric case, which by itself will completely specify the transformation parameters $b_{11}$ and $b_{22}$. Subsequently, we can then generalize to include parity-non symmetric situations, although we defer from carrying out this explicitly here. It will turn out that the task of analysing this set of seven coupled inequalities is considerably simplified by going over to new parameters, as defined in table 4 . Also, it will turn out to be convenient in the cases II and III to replace $b_{11}$ and $b_{22}$ by $q_{1,2}$ as follows 


$$
b_{11}=q_{1} \quad, \quad b_{22}=\left\{\begin{array}{cc}
S q_{2} & ; \text { case II } \\
1-b_{11}+S q_{2} & ; \text { case III }
\end{array}\right.
$$

\subsection{Calculation of the stochastic rates}

We now give the rates of the stochastic system as obtained from the integrable chain $H$.

\subsubsection{Case I: integrability through equations of motion}

After performing the similarity transformation (4.2) on the matrix

$$
H^{I}=\left(\begin{array}{cccc}
h_{11} & h_{12} & h_{13} & h_{14} \\
0 & h_{22} & h_{23} & h_{24} \\
0 & h_{32} & h_{33} & h_{34} \\
0 & 0 & 0 & 0
\end{array}\right)
$$

with $B$ given by $(4.15)$ we obtain the transformed matrix $\widetilde{H^{I}}$. The probability conservation condition (2.8), expressed, by eqs. (3.7) are here automatically satisfied and we have only to worry about the positivity conditions of the elements $\widetilde{h}_{i j}^{I}$ for all $i \neq j$.

In this case, however, it turns out that the transformed elements $\widetilde{h}_{i j}^{I}$ do satisfy the following pair of equations:

$$
\begin{aligned}
& D_{1}^{I}:=\widetilde{h}_{34}^{I}-\widetilde{h}_{21}^{I}-\widetilde{h}_{41}^{I}-\widetilde{h}_{12}^{I}-\widetilde{h}_{32}^{I}+\widetilde{h}_{23}^{I}+\widetilde{h}_{43}^{I}+\widetilde{h}_{14}^{I}=0 \\
& D_{2}^{I}:=\widetilde{h}_{24}^{I}-\widetilde{h}_{31}^{I}-\widetilde{h}_{41}^{I}-\widetilde{h}_{13}^{I}-\widetilde{h}_{23}^{I}+\widetilde{h}_{32}^{I}+\widetilde{h}_{42}^{I}+\widetilde{h}_{14}^{I}=0
\end{aligned}
$$

When these conditions are both satisfied, one has a closed system of $k$ equations for the equal-time $k$-point correlation functions [25]. Since it can be checked immediately from the rates of the stochastic system whether or not the conditions eqs. (4.32) are satisfied, the description either through an integrable quantum chain or through the equations of motion offer the same amount of information. While it might be easier to extract relaxation times from the quantum Hamiltonian, the full time-dependence of observables like the density is probably more easily extracted from the equations of motion.

For practical applications it might be useful to repeat eqs. (4.32) where the $\widetilde{h}_{i j}$ are replaced by the physical rates

$$
\begin{aligned}
\widetilde{\beta}_{R}+\widetilde{\gamma}_{L}+2 \widetilde{\alpha}+\widetilde{D}_{R} & =\widetilde{\delta}_{R}+\widetilde{\sigma}_{R}+2 \widetilde{\nu}+\widetilde{D}_{L} \\
\widetilde{\beta}_{L}+\widetilde{\gamma}_{R}+2 \widetilde{\alpha}+\widetilde{D}_{L} & =\widetilde{\delta}_{L}+\widetilde{\sigma}_{L}+2 \widetilde{\nu}+\widetilde{D}_{R}
\end{aligned}
$$

The two conditions coincide in the parity-symmetric case when they read

$$
\widetilde{\delta}+\widetilde{\sigma}+2 \widetilde{\nu}=\widetilde{\beta}+\widetilde{\gamma}+2 \widetilde{\alpha}
$$


and we note that the value of the diffusion constant $\widetilde{D}$ does not enter into the integrability condition.

\subsubsection{Case II}

The matrix to be transformed is now

$$
H^{I I}=\left(\begin{array}{cccc}
0 & h_{12} & -h_{12} & 0 \\
0 & h_{22} & h_{23} & h_{24} \\
0 & h_{32} & h_{33} & h_{34} \\
0 & 0 & 0 & h_{44}
\end{array}\right)
$$

and the transformation matrix $B$ is given by (4.18). Probability conservation is already implemented. In view of the result of the previous subsection, it is of interest to check for integrablity through the equations of motion. The expressions from eqs. (4.32) become in this case:

$$
\begin{aligned}
& D_{1}^{I I}=-\left(h_{12}+h_{24}\right) /\left(S q_{2}\right) \\
& D_{2}^{I I}=\left(h_{12}-h_{34}\right) /\left(S q_{2}\right)
\end{aligned}
$$

and we see that the integrability from case $\mathrm{I}$ is only recovered if simultaneously $\Omega=0$ and $h_{12}=h_{34}$ (provided $q_{2}$ remains finite).

Using the notation of table 1 , we can now write down the parity-symmetric rates

$$
\begin{aligned}
\widetilde{\alpha} & =-\frac{P}{2 q_{2}} q_{1}^{2}\left(q_{2}-q_{1}\right) \\
\widetilde{\beta} & =\frac{P}{q_{2}}\left(q_{1}-1\right)\left[q_{1}\left(q_{1}-1\right)-q_{2}\left(q_{1}+R\right)\right] \\
\widetilde{\gamma} & =-\frac{P}{q_{2}} q_{1}\left[q_{1}\left(q_{1}-\frac{1}{2}\right)-q_{2}\left(q_{1}+R\right)\right] \\
\widetilde{\delta} & =\frac{P}{q_{2}} q_{1}\left[q_{1}\left(q_{1}-1\right)-q_{2}\left(q_{1}-1-R\right)\right] \\
\widetilde{\nu} & =-\frac{P}{2 q_{2}}\left(q_{1}-1\right)^{2}\left(q_{2}+1-q_{1}\right) \\
\widetilde{\sigma} & =-\frac{P}{q_{2}}\left(q_{1}-1\right)\left[\left(q_{1}-\frac{1}{2}\right)\left(q_{1}-1\right)-q_{2}\left(q_{1}-1-R\right)\right] \\
\widetilde{D} & =-\frac{P}{q_{2}}\left[q_{1}\left(q_{1}-\frac{1}{2}\right)\left(q_{1}-1\right)-q_{2} q_{1}\left(q_{1}-1\right)+\frac{1}{2} q_{2} \Delta\right]
\end{aligned}
$$

We remark that the parameters are defined such that $P$ always emerges as a prefactor. This implies of course that $P \neq 0$ and we shall consider later what happens when $P=0$. In order to get a stochastic system, the seven rates of eq. (4.37) must all be positive. We postpone the analysis of these inequalities to the next section and discuss first the rates for case III. 


\subsubsection{Case III}

The matrix to be transformed is

$$
H^{I I I}=\left(\begin{array}{cccc}
0 & h_{12} & -h_{12}-h_{22}-h_{33}-h_{23}-h_{32} & -h_{24}-h_{34}-h_{44} \\
0 & h_{22} & h_{23} & h_{24} \\
0 & h_{32} & h_{33} & h_{34} \\
0 & 0 & 0 & h_{44}
\end{array}\right)
$$

and the transformation matrix $B$ is given by (4.29). Again, eqs. (3.7) are automatically satisfied with $\widetilde{C_{1}}=\widetilde{C_{4}}=0$ and $\widetilde{C_{2}}=-\widetilde{C_{3}}=h_{22}+h_{32}+h_{12}$. We begin by looking at the expressions $D_{1,2}^{I I I}$, see (4.32), which for integrability through the equation of motions for the particle number correlators whould have to vanish and which become in this case

$$
\begin{aligned}
& D_{1}^{I I I}=\left(h_{23}-h_{32}-h_{44}-h_{12}-h_{24}\right) /\left(S q_{2}\right) \\
& D_{2}^{I I I}=\left(h_{22}+2 h_{32}+h_{33}+h_{12}-h_{44}-h_{34}\right) /\left(S q_{2}\right)
\end{aligned}
$$

Now, we observe that with the parametrization of table 1 and eq. (4.30), the expression for rates in the parity-symmetric case are exactly the same as in case II and already given in eqs. (4.37). Therefore for the analysis of the positivity, it is enough to consider one of the cases only. As a bonus, we shall afterwards have the freedom to work with the integrable matrices $H$ of either case II or case III, depending of the applications of interest.

We stress, however, that the coincidence of the rates for the cases II and III only holds when $P \neq 0$. When $P=0$, both cases must be discussed separately.

\subsection{Analysis of the positivity conditions}

We now adress the problem of how to characterize those stochastic systems which can be transformed via (2.19) to the integrable Hamiltonian $H^{I I}$ or $H^{I I I}$. We restrict attention to the case when the elements of $H$ as well as the transformation parameters $q_{1,2}$ are real. ${ }^{\text {If }}$ We thus have to satisfy simultaneously the seven inequalities

$$
\widetilde{\alpha} \geq 0, \widetilde{\beta} \geq 0, \widetilde{\gamma} \geq 0, \widetilde{\delta} \geq 0, \widetilde{\nu} \geq 0, \widetilde{\sigma} \geq 0, \widetilde{D} \geq 0
$$

and the rates are given explicitly in eqs. (4.37). Since the parameter $\Delta$ appears only in $\widetilde{D}$, we can always satisfy the corresponding inequality by suitably choosing $\Delta$ and it remains to consider the other six inequalities.

\footnotetext{
${ }^{14}$ We had already seen in the discussion of case III above that this condition might be the one needed for applications anyway. In addition our results from section 3, where all the elements of the free fermion Hamiltonian turned out to be real at the end makes it plausible that this should be the most important case.
} 
While at first sight this problem looks rather difficult, it can be solved, following the ideas presented in section 3 after having made a few observations. From eqs. (4.37), we first note that $P / q_{2}$ only appears as a scale factor and thus only its sign is going to be relevant for the positivity discussion. Second, after rescaling the rates depend only on the transformation parameters $q_{1}$ and $q_{2}$, which characterize the similarity transformation $B$, and the parameter $R$, which characterizes the Hamiltonian $H$. We think of the rates as functions of these three parameters, e.g. $\widetilde{\alpha}=\widetilde{\alpha}\left(q_{1}, q_{2}, R\right)$. We now fix $R$ and look at the values of the function $\widetilde{\alpha}$ in $q_{1}-q_{2}$ space. There will be regions $G_{+}^{(\alpha)}$ for which $\widetilde{\alpha}$ will be positive and regions for which $\widetilde{\alpha}$ will be negative. The boundaries of the regions $G_{+}^{(\alpha)}$ are characterized by the curves $\widetilde{\alpha}\left(q_{1}, q_{2} ; R\right)=0$. Mapping out the boundary curves for a fixed value of $R$ will rapidly furnish a geometrical idea of the shape of the region $G_{+}^{(\alpha)}$. This process is repeated for all six rates and the finally the desired region in $q_{1}-q_{2}$ space where all rates are positive is the intersection

$$
G_{+}=G_{+}^{(\alpha)} \cap G_{+}^{(\beta)} \cap G_{+}^{(\gamma)} \cap G_{+}^{(\delta)} \cap G_{+}^{(\nu)} \cap G_{+}^{(\sigma)}
$$

The graphical inspection of these regions will then suggest how to satisfy in a simple way the inequalities (4.40). Technically, the proof is much shortened by concentrating on the case with a single steady state which makes the inequalities (4.20) available. Then the following distinction is sensible: $i$ ) $q_{1}=0$, ii) $q_{1}=1$ and $\left.i i i\right) 0<q_{1}<1$. (If eq. (4.20) does not apply, it can be shown that no further stochastic systems exist.)

\subsection{1 $\quad q_{1}=0$}

In this case the parity-symmetric rates become

$$
\begin{aligned}
& \widetilde{\alpha}=\widetilde{\gamma}=\widetilde{\delta}=0, \widetilde{\beta}=P R, \quad \widetilde{\nu}=-\frac{P}{2 q_{2}}\left(q_{2}+1\right) \\
& \widetilde{\sigma}=\frac{P}{2 q_{2}}\left(2 q_{2}(1+R)+1\right), \widetilde{D}=-\frac{1}{2} P \Delta
\end{aligned}
$$

It is easy to see that for these rates, the positivity conditions can be satisfied. However, the integrability of this model is physically trivial, since only particle creation reactions are allowed.

\subsection{2 $q_{1}=1$}

In this case the parity-symmetric rates become

$$
\begin{aligned}
& \widetilde{\beta}=\widetilde{\nu}=\widetilde{\sigma}=0, \quad \widetilde{\alpha}=\frac{P}{2 q_{2}}\left(1-q_{2}\right), \quad \widetilde{\gamma}=\frac{P}{2 q_{2}}\left(2 q_{2}(1+R)-1\right) \\
& \widetilde{\delta}=P R, \widetilde{D}=-\frac{1}{2} P \Delta
\end{aligned}
$$


The discussion of positivity is completely analogous to the case above. Again, since only particle annihilation reactions are allowed, the integrability of the system is trivial and we just recover the model reviewed in section 2 .

\subsection{3 $0<q_{1}<1$}

We now turn to the discussion of the remaining cases. Again, the different possibilities for the signs of $P$ and $q_{2}$ must be treated separately.

1. $P=-1$ and $q_{2}>0$. From the positivity of the rates, provided that neither $q_{1}=0$ nor $q_{1}=1$, we get the following inequalities

$$
\begin{aligned}
& \widetilde{\alpha} \geq 0 \quad \Longrightarrow \quad q_{1}-q_{2} \leq 0 \\
& \widetilde{\beta} \geq 0 \quad \Longrightarrow \quad q_{1}\left(q_{1}-1\right)-q_{2}\left(q_{1}+R\right) \geq 0 \\
& \widetilde{\gamma} \geq 0 \quad \Longrightarrow \quad q_{1}\left(q_{1}-\frac{1}{2}\right)-q_{2}\left(q_{1}+R\right) \geq 0 \\
& \widetilde{\delta} \geq 0 \quad \Longrightarrow q_{1}\left(q_{1}-1\right)-q_{2}\left(q_{1}-1-R\right) \leq 0 \\
& \widetilde{\nu} \geq 0 \quad \Longrightarrow \quad q_{1}-q_{2}-1 \leq 0 \\
& \widetilde{\sigma} \geq 0 \quad \Longrightarrow\left(q_{1}-\frac{1}{2}\right)\left(q_{1}-1\right)-q_{2}\left(q_{1}-1-R\right) \leq 0 \\
& \widetilde{D} \geq 0 \quad \Longrightarrow \quad q_{1}\left(q_{1}-\frac{1}{2}\right)\left(q_{1}-1\right)-q_{2}\left(q_{1}\left(q_{1}-1\right)-\Delta / 2\right) \geq 0
\end{aligned}
$$

From these, we observe that

$$
R \leq-\frac{1}{2}
$$

To see this, we note that from $\widetilde{\beta} \geq 0$ and $\widetilde{\delta} \geq 0$ we have the inequalities $q_{1}\left(q_{1}-1\right) \geq q_{2}\left(q_{1}+R\right)$ and $q_{1}\left(q_{1}-1\right) \leq q_{2}\left(q_{1}-1-R\right)$, respectively. Combining these, we find that $q_{2}\left(q_{1}+R\right) \leq q_{2}\left(q_{1}-1-R\right)$ and since $q_{2}>0$, the assertion follows. Next, we have the following

Lemma 1: If $P=-1$ and $q_{2}>0$ and $0<q_{1}<1$, the conditions

$$
\widetilde{\beta} \geq 0, \widetilde{\nu} \geq 0, \widetilde{\sigma} \geq 0, \widetilde{D} \geq 0
$$

are sufficient for positivity.

Proof: Since $0<q_{1}<1$, we have $q_{1}\left(q_{1}-1\right) \leq\left(q_{1}-1 / 2\right)\left(q_{1}-1\right)$. Now, $\widetilde{\sigma} \geq 0$ and $\tilde{\delta} \geq 0$ are equivalent to the inequalities $\left(q_{1}-1 / 2\right)\left(q_{1}-1\right) \leq q_{2}\left(q_{1}-1-R\right)$ and $\left(q_{1}-1\right) q_{1} \leq q_{2}\left(q_{1}-1-R\right)$, respectively. It is now obvious that $\widetilde{\sigma} \geq 0$ implies $\widetilde{\delta} \geq 0$. On the other hand, we also have the inequality $q_{1}\left(q_{1}-1 / 2\right) \geq q_{1}\left(q_{1}-1\right)$. Now, $\widetilde{\beta} \geq 0$ and $\widetilde{\gamma} \geq 0$ are equivalent to the inequalities $q_{1}\left(q_{1}-1\right) \geq q_{2}\left(q_{1}+R\right)$ and $q_{1}\left(q_{1}-1 / 2\right) \geq q_{2}\left(q_{1}+R\right)$, respectively. It follows that $\widetilde{\beta} \geq 0$ implies that $\widetilde{\gamma} \geq 0$. Finally, $\widetilde{\alpha} \geq 0$ 
and $\widetilde{\nu} \geq 0$ are equilavent to the inequalities $q_{1} \leq q_{2}$ and $q_{1} \leq q_{2}+1$, respectively, which completes the proof.

q.e.d.

It remains to characterize $G_{+}$from the remaining rates. The result is

$$
G_{+, 1}=\left\{\left(q_{1}, q_{2}, R\right) \mid 1+R \leq q_{1} \leq-R ; q_{2} \geq \max \left(\frac{q_{1}\left(q_{1}-1\right)}{q_{1}+R}, \frac{\left(q_{1}-1 / 2\right)\left(q_{1}-1\right)}{q_{1}-1-R}, q_{1}-1\right) ; R \leq-\frac{1}{2}\right\}
$$

2. $P=-1$ and $q_{2}<0$. Now we have the following set of inequalities

$$
\begin{aligned}
& \widetilde{\alpha} \geq 0 \quad \Longrightarrow \quad q_{1}-q_{2} \geq 0 \\
& \widetilde{\beta} \geq 0 \quad \Longrightarrow \quad q_{1}\left(q_{1}-1\right)-q_{2}\left(q_{1}+R\right) \leq 0 \\
& \widetilde{\gamma} \geq 0 \quad \Longrightarrow \quad q_{1}\left(q_{1}-\frac{1}{2}\right)-q_{2}\left(q_{1}+R\right) \leq 0 \\
& \widetilde{\delta} \geq 0 \quad \Longrightarrow q_{1}\left(q_{1}-1\right)-q_{2}\left(q_{1}-1-R\right) \geq 0 \\
& \widetilde{\nu} \geq 0 \quad \Longrightarrow \quad q_{1}-q_{2}-1 \geq 0 \\
& \widetilde{\sigma} \geq 0 \quad \Longrightarrow\left(q_{1}-\frac{1}{2}\right)\left(q_{1}-1\right)-q_{2}\left(q_{1}-1-R\right) \geq 0 \\
& \widetilde{D} \geq 0 \quad \Longrightarrow q_{1}\left(q_{1}-\frac{1}{2}\right)\left(q_{1}-1\right)-q_{2}\left(q_{1}\left(q_{1}-1\right)-\Delta / 2\right) \leq 0
\end{aligned}
$$

The treatment is completely analogous to the first case. Again, from $\widetilde{\beta}$ and $\widetilde{\delta}$ we find that $R \leq-\frac{1}{2}$. Then we prove the

Lemma 2: If $P=-1$ and $q_{2}<0$ and $0<q_{1}<1$, the conditions

$$
\widetilde{\alpha} \geq 0, \widetilde{\gamma} \geq 0, \widetilde{\delta} \geq 0, \widetilde{D} \geq 0
$$

are sufficient for positivity.

Finally, the region $G_{+}$where all rates are positive is found to be

$$
G_{+, 2}=\left\{\left(q_{1}, q_{2}, R\right) \mid 1+R \leq q_{1} \leq-R ; q_{2} \leq \min \left(\frac{q_{1}\left(q_{1}-1 / 2\right)}{q_{1}+R}, \frac{q_{1}\left(q_{1}-1\right)}{q_{1}-1-R}, q_{1}\right) ; R \leq-\frac{1}{2}\right\}
$$

3. $P=+1$ and $q_{2}>0$. In fact this case does not give anything new. By assumption we have $0<q_{1}<1$. From $\widetilde{\nu} \geq 0$ it follows that $0<q_{2} \leq q_{1}-1<0$, which is impossible, because $q_{2}=0$ would correspond to a singular transformation matrix $B$.

4. $P=+1$ and $q_{2}<0$. Also here no new system arises. From $\widetilde{\alpha} \geq 0$ it follows that $0<q_{1} \leq q_{2}<0$ which is impossible. The only way to find stochastic systems for $P$ positive is to consider the cases $q_{1}=0,1$ which have been dealt with before.

In conclusion, the only non-trivial parity-symmetric stochastic system which can be reduced through (2.19) to a real integrable quantum chain with the spectrum of the XXZ Hamiltonian is the class of 
models with rates given in eq. (4.37) and the domain of positivity

$$
G_{+}=G_{+, 1} \cup G_{+, 2}
$$

We remark that in the $q_{1}-q_{2}$ plane, the regions $G_{+, 1}$ and $G_{+, 2}$ are mapped onto each other under the transformation $\left(q_{1}, q_{2}\right) \rightarrow\left(1-q_{1},-q_{2}\right)$, or geometrically speaking, by point symmetry through the point $q_{1}=\frac{1}{2}, q_{2}=0$.

The positivity condition in (4.51) is supplemented, for $P=-1$, but independently of the sign of $q_{2}$, by the inequality

$$
\Delta \geq 2 q_{1}\left(q_{1}-1\right) \frac{q_{2}-q_{1}+1 / 2}{q_{2}}
$$

Eqs. (4.51,4.52) specify completely, for the non-trivial case $P=-1$, the restrictions which have to be put on the values of the free parameters $q_{1}, q_{2}, R, \Delta$.

\subsubsection{The case $P=0$}

We complete the analysis by stating the results in the case when $P=0$. For the proofs, we refer the reader to appendix D.

Case II: Here positivity requires that $\Omega=Q=0$ and consequently, all reaction rates vanish. The only processes which survive are diffusion to the right and to the left.

Case III: If there is just a single steady state, we find the stochastic system

$$
\widetilde{H}_{j, j+1}=\frac{1}{2}\left(\begin{array}{cccc}
g_{1} & -b_{11} h_{44} & -b_{11} h_{44} & 0 \\
\left(b_{11}-1\right) h_{44} & g_{2} & \left(h_{23}+h_{32}\right) & -b_{11} h_{44} \\
\left(b_{11}-1\right) h_{44} & \left(h_{23}+h_{32}\right) & g_{3} & -b_{11} h_{44} \\
0 & \left(b_{11}-1\right) h_{44} & \left(b_{11}-1\right) h_{44} & g_{4}
\end{array}\right)
$$

where the $g_{\ell}$ are determined such that the sum of each of the column vector of $\widetilde{H}_{j, j+1}$ vanishes. From a physical point of view, however, the integrability of the model is obvious, since it describes particles moving diffusively and, since $\widetilde{\gamma}=\widetilde{\delta}=r_{-}$and $\widetilde{\beta}=\widetilde{\sigma}=r_{+}$, decay radioactively with rate $r_{-}$and are created independently of their neighbors with rate $r_{+}$. We remark that in this case, it is easily checked that the integrability conditions through the equations of motion, viz. $D_{1}^{I I I}=D_{2}^{I I I}=0$, are satisfied.

\subsection{Some applications}

We are now in a position to give a more physical characterization of the new integrable stochastic systems obtained. We shall discuss here only the class of models of which the rates are explicitly given in (4.37) with the domain $G_{+}$(4.51), together with (4.52), gives the range of variables where the 
stochastic rates are positive, and shall leave out completely all the other systems, since their behaviour is easily obtained without reference to the XXZ chain.

Let us begin with a discussion of the meaning of the condition $R \leq-\frac{1}{2}$. Recalling the definition of $R$ from table $⿴$ t and the fact that $P$ must be negative, we get the inequality $Q \geq-P=Q-h_{44}$, from which it follows that both $h_{44} \geq 0$ and $Q>h_{44}$ are necessarily satisfied. These are also necessary conditions for $H$ to be a stochastic matrix by itself. In order to understand eq. (4.45) further, we consider the example of $H^{I I I}$ in the parity-symmetric case and we also assume that $H^{I I I}$ is itself stochastic. Then the matrix elements of $H$ can be written in terms of chemical reaction rates, using the notation of table 田as

$$
h_{22}=h_{33}=D+\delta, h_{23}=h_{32}=D, h_{44}=2(\alpha+\gamma)
$$

We then find that $Q=2 \delta$ and $P=2(\alpha+\gamma+\delta)$ and finally

$$
R=\frac{Q}{2 P}=-\frac{1}{2} \frac{\delta}{\delta-\alpha-\gamma} \leq-\frac{1}{2}
$$

because $Q>h_{44}$ implies $\delta>\alpha+\gamma$. So we see that in this particular example eq. (4.45) simply means that also $H$ is stochastic.

Next, we ask for a characterization of integrability through similarity transformation to the XXZ chain solely in terms of the chemical reaction rates. In eqs. 4.37), the six rates (without the diffusion constant $\widetilde{D}$ ) are expressed in terms of the four parameters $q_{1}, q_{2}, P$ and $R$. So we should expect to find two relations between the rates. Indeed, tedious calculations lead to the following relations

$$
\begin{aligned}
& \left\{-4 \widetilde{\nu}^{3}+[8 \widetilde{\delta}+4 \widetilde{\beta}-12 \widetilde{\sigma}] \widetilde{\nu}^{2}+[(8 \widetilde{\sigma}-2 \widetilde{\beta}) \widetilde{\delta}-(\widetilde{\beta}-2 \widetilde{\sigma})(\widetilde{\beta}-6 \widetilde{\sigma})] \widetilde{\nu}-\widetilde{\sigma}(\widetilde{\beta}-2 \widetilde{\sigma})^{2}\right\} \widetilde{\alpha} \\
+ & 4 \widetilde{\alpha}^{2} \widetilde{\nu}^{2}-\widetilde{\delta}^{2} \widetilde{\nu}^{2}+\left(2 \widetilde{\delta}^{3}+(\widetilde{\beta}-2 \widetilde{\sigma}) \widetilde{\delta}^{2}\right) \widetilde{\nu}+\widetilde{\sigma}(-\widetilde{\sigma}+\widetilde{\beta}) \widetilde{\delta}^{2}=0
\end{aligned}
$$

and

$$
\begin{gathered}
\left\{-4 \widetilde{\nu}^{3}+[4 \widetilde{\gamma}+4 \widetilde{\delta}-12 \widetilde{\sigma}] \widetilde{\nu}^{2}+2(\widetilde{\gamma}-2 \widetilde{\sigma}) \widetilde{\sigma}^{2}+\left[\widetilde{\delta}^{2}+4 \widetilde{\delta} \widetilde{\sigma}+6(\widetilde{\gamma}-2 \widetilde{\sigma}) \widetilde{\sigma}\right] \widetilde{\nu}\right\} \widetilde{\alpha} \\
+\widetilde{\gamma}^{2} \widetilde{\sigma}^{2}-\widetilde{\delta}^{2} \widetilde{\sigma}^{2}+\left(4 \widetilde{\nu}^{2}+4 \widetilde{\nu} \widetilde{\sigma}+\widetilde{\sigma}^{2}\right) \widetilde{\alpha}^{2}+\left(\widetilde{\gamma}^{2}-\widetilde{\delta}^{2}\right) \widetilde{\nu}^{2}+\left(2 \widetilde{\gamma}^{2} \widetilde{\sigma}+\widetilde{\delta}^{3}+(\widetilde{\gamma}-2 \widetilde{\sigma}) \widetilde{\delta}^{2}\right) \widetilde{\nu}=0
\end{gathered}
$$

While in case I, the integrability condition could be expressed as a linear relation (4.34) between the rates, we have here two quartic relations. We note that, as in case I, the value of the diffusion constant $\widetilde{D}$ does not enter into the integrability conditions. For a given reaction-diffusion system, eqs. (4.56,4.57) can be used to check whether or not the model is integrable through the transformation into an XXZ chain. 
Since the type of integrability we consider here mainly involves the spectrum of the Hamiltonian $H$, it is particularly easy to extract the relaxation time towards the steady state and the mean particle density in the steady state. The relaxation time $\tau$ is the inverse of the mass gap $Q$ and thus given by

$$
\frac{1}{\tau}=Q=2 P R
$$

In particular, since both $P$ and $R$ are negative, it follows that the relaxation time $\tau$ is always finite. A tedious calculation gives $\tau$ in terms of the reaction rates

$$
\begin{aligned}
\tau^{-1} & =-2 \frac{(-2 \widetilde{\sigma}-6 \widetilde{\nu}+\widetilde{\beta}) \widetilde{\alpha}-2 \widetilde{\nu}^{2}+(\widetilde{\beta}-4 \widetilde{\sigma}) \widetilde{\nu}-2 \widetilde{\delta} \widetilde{\sigma}+\widetilde{\beta} \widetilde{\sigma}-2 \widetilde{\sigma}^{2}-\widetilde{\delta}^{2}}{(-2 \widetilde{\sigma}-4 \widetilde{\nu}+\widetilde{\beta}) \widetilde{\alpha}-\widetilde{\delta} \widetilde{\nu}-\widetilde{\delta}(\widetilde{\delta}+\widetilde{\sigma})} \\
& \times \frac{\left(\left(-8 \widetilde{\nu}^{2}+(-12 \widetilde{\sigma}+2 \widetilde{\beta}+2 \widetilde{\delta}) \widetilde{\nu}+2 \widetilde{\sigma}(-2 \widetilde{\sigma}+\widetilde{\beta})\right) \widetilde{\alpha}-2 \widetilde{\delta}(3 \widetilde{\delta}+\widetilde{\beta}) \widetilde{\nu}-2 \widetilde{\delta} \widetilde{\sigma}(\widetilde{\delta}+\widetilde{\beta})\right)}{\left(2 \widetilde{\alpha} \widetilde{\nu}+2 \widetilde{\nu}^{2}+(-\widetilde{\beta}+4 \widetilde{\sigma}-\widetilde{\delta}) \widetilde{\nu}-\widetilde{\sigma}(-2 \widetilde{\sigma}-\widetilde{\delta}+\widetilde{\beta})\right)}
\end{aligned}
$$

We remark that in this form there is no longer an explicit dependence on neither the coagulation rate $\widetilde{\gamma}$ nor the diffusion constant $\widetilde{D}$.

Finally, the mean particle density in the steady state is found from $\widetilde{\rho}_{\infty}=q_{1}$. In terms of the rates, it reads

$$
\widetilde{\rho}_{\infty}=\frac{(-2 \widetilde{\sigma}-4 \widetilde{\nu}+\widetilde{\beta}) \widetilde{\alpha}-\widetilde{\delta} \widetilde{\nu}-\widetilde{\delta}(\widetilde{\delta}+\widetilde{\sigma})}{(-2 \widetilde{\sigma}-6 \widetilde{\nu}+\widetilde{\beta}) \widetilde{\alpha}-2 \widetilde{\nu}^{2}+(\widetilde{\beta}-4 \widetilde{\sigma}) \widetilde{\nu}-\widetilde{\delta} \widetilde{\delta}^{2}-2 \widetilde{\delta} \widetilde{\sigma}+\widetilde{\beta} \widetilde{\sigma}-2 \widetilde{\sigma}^{2}}
$$

which is also not explicitly dependent on $\widetilde{\gamma}$ or $\widetilde{D}$.

\subsection{Summary}

In this section, we have found the parity-symmetric stochastic processes which are integrable through a similarity transformation of the type (2.19) onto a real XXZ quantum chain. Up to rescaling, we find a non-trivial class of integrable systems, which depends on three parameters. These systems approach their steady steady state exponentially fast. Their rates are explicitly given in eqs. (4.37) and the constraints on the free parameters $q_{1,2}, P$ and $R$ are given in (4.51). Since, working within the setting of case III, the integrable model is for the parity-symmetric case identical to the known system eq. (2.21), we can read off from table 2 the time dependence of one- and two-point correlators in the $t \rightarrow \infty$ limit. 


\section{Calculation of the density for uncorrelated initial states with translational invariance}

We will now illustrate the use of the similarity transformations developed in the previous sections. We will calculate the mean density $\tilde{\rho}(t)$ for the various cases presented in table 3 for an uncorrelated initial state with density $\tilde{\rho}_{0}$. Since the mean density $\widetilde{\rho}$ is translation invariant, the result will not depend on the diffusion bias $D_{L}-D_{R}$. Since the bias is proportional to $D_{1}-D_{2}$, it is enough to set $D_{1}=D_{2}=1$, which fixes the time scale as well, see eq. (3.12). This calculation is greatly simplified using the free fermion formulation given by the Hamiltonian eq. (3.1).

The initial state is of the product form

$$
\left|\tilde{\rho}_{0}\right\rangle=\bigotimes_{j=1}^{L}\left(\begin{array}{c}
1-\tilde{\rho}_{0} \\
\tilde{\rho}_{0}
\end{array}\right)_{j} .
$$

We wish to calculate the matrix element

$$
\left\langle s\left|\tilde{n}_{x} \exp (-\tilde{H} t)\right| \tilde{\rho}_{0}\right\rangle=\left\langle s\left|B n_{x} \exp (-H t) B^{-1}\right| \tilde{\rho}_{0}\right\rangle
$$

averaged over all sites. In section 3, we had already calculated $n_{x}$ for the four systems of table 3 and related the parameters of the stochastic Hamiltonians to the parameters of the free fermion Hamiltonian (3.1). Since the transformation $(2.19)$ is local and $\left|\widetilde{\rho}_{0}\right\rangle$ is a product state, the state $B^{-1}\left|\tilde{\rho}_{0}\right\rangle$ is also a product state. As pointed out in the previous section, the normalization of a state is arbitrary and therefore we can always represent $\left|\rho_{0}\right\rangle=B^{-1}\left|\tilde{\rho}_{0}\right\rangle$ in the form (5.1) with $\tilde{\rho}_{0}$ substituted by $\rho_{0}$. Also, $\langle s| B=\langle\omega|$, where $\langle\omega|=\bigotimes_{j=1}^{L}(\omega, 1-\omega)$. The ratios $\frac{\rho_{0}}{1-\rho_{0}}$ and $\frac{1-\omega}{\omega}$ can be found for each separate case of section 3 by applying $B^{-1}$ to $\left|\tilde{\rho}_{0}\right\rangle$ and $B$ to $\langle s|$.

The operator $n_{x}$ was written for models I-III in terms of the Pauli spin operators $\sigma_{x}^{y}$ and $\sigma_{x}^{z}$ in section 3 (for model IV, trivially $n_{x}=\tilde{n}_{x}$ ). Due to the form of $\langle\omega|$ we have that

$$
\langle\omega| \sigma_{x}^{y}=\mathrm{i} \frac{1-\omega}{\omega}\langle\omega|\left(1-\tilde{n}_{x}\right)-\mathrm{i} \frac{\omega}{1-\omega}\langle\omega| \tilde{n}_{x}
$$

(if $\omega \neq 0,1$ ). Furthermore, $\sigma_{x}^{z}=1-2 \tilde{n}_{x}$. Therefore the calculation of the density is reduced to the calculation of the matrix element $\sum_{x}\left\langle\omega\left|\tilde{n}_{x} \exp (-H t)\right| \rho_{0}\right\rangle$. In order to do this, we write the Hamiltonian (3.1) in terms of spinless fermion operators by means of a Jordan-Wigner transformation, see appendix A. Then we express the fermion creation and annihilation operators at a given site $x$ in terms of their discrete Fourier transforms to momentum space. As is well known from the properties of the Jordan-Wigner transformation, we have antiperiodic (periodic) boundary conditions in the sector 
with an even (odd) number of particles, e.g. 67, 55, 61]. In terms of the spin language, each new fermion corresponds to a spin flip, the ferromagnetic state with all spins up being the fermion vacuum. For simplicity, we shall work in the even sector, since this simplification does not affect the results in the thermodynamic limit. The Hamiltonian (3.1) becomes

$$
H=(1-h) L+\sum_{p>0}\left(2 h-2 \cos \left(\frac{2 \pi p}{L}\right)\right)\left(\hat{b}_{p}^{\dagger} \hat{b}_{p}+\hat{b}_{-p}^{\dagger} \hat{b}_{-p}\right)-2 \sin \left(\frac{2 \pi p}{L}\right)\left(\eta_{2} \hat{b}_{-p}^{\dagger} \hat{b}_{p}^{\dagger}+\eta_{1} \hat{b}_{p} \hat{b}_{-p}\right)
$$

where $p$ runs over the half-integers $1 / 2, \ldots, L / 2-1 / 2$ because of the antiperiodic boundary conditions. The number operator is written in terms of fermion operators as $\sum_{x} \tilde{n}_{x}=\sum_{p} \hat{b}_{p}^{\dagger} \hat{b}_{p}$.

Finally it can be shown [55, 68] that the projection of the states $\langle\omega|$ and $\left|\rho_{0}\right\rangle$ over the even sector can be written as

$$
\left\langle\left.\omega\right|^{e v e n}=\langle 0| \prod_{p>0}\left(1+f_{\omega}^{2} \cot \left(\frac{\pi p}{L}\right) \hat{b}_{p} \hat{b}_{-p}\right)\right.
$$

with $f_{\omega}=\frac{1-\omega}{\omega}$ and

$$
\left|\rho_{0}\right\rangle^{\text {even }}=\mathcal{N}^{-1} \prod_{p>0}\left(1+\mu^{2} \cot \left(\frac{\pi p}{L}\right) \hat{b}_{-p}^{\dagger} \hat{b}_{p}^{\dagger}\right)|0\rangle
$$

where $\mu=\rho_{0} /\left(1-\rho_{0}\right)$ and the normalization $\mathcal{N}=\left\langle\omega \mid \rho_{0}\right\rangle^{\text {even }}$.

So we have reduced our problem to the calculation of $\left\langle\left.\omega\right|^{\text {even }} \hat{b}_{p}^{\dagger} \hat{b}_{p} \exp (-H t) \mid \rho_{0}\right\rangle^{\text {even }}$. Notice that both states $\left\langle\left.\omega\right|^{\text {even }} \text { and } \mid \rho_{0}\right\rangle^{\text {even }}$ belong to a translation invariant subspace of the original Hilbert space with zero momentum. In this subspace, the operators $\hat{b}_{p} \hat{b}_{-p}, \hat{b}_{-p}^{\dagger} \hat{b}_{p}^{\dagger}$ and $1-\hat{b}_{p}^{\dagger} \hat{b}_{p}-\hat{b}_{-p}^{\dagger} \hat{b}_{-p}$ are isomorphic to a Pauli spin algebra [68], corresponding respectively to $\sigma_{p}^{+}, \sigma_{p}^{-}$and $\sigma_{p}^{z}$. One can therefore develop the term $\exp (-H t)$ using the known rules for the exponential of an expression involving Pauli spin matrices. First notice that

$$
\sum_{p}\left\langle\left.\omega\right|^{\text {even }} \hat{b}_{p}^{\dagger} \hat{b}_{p} \exp (-H t) \mid \rho_{0}\right\rangle^{\text {even }}=2 \sum_{p>0} f_{\omega}^{2} \cot \left(\frac{\pi p}{L}\right)\left\langle\left.\omega\right|^{\text {even }} \hat{b}_{p} \hat{b}_{-p} \exp (-H t) \mid \rho_{0}\right\rangle^{\text {even }}
$$

since

$$
\left\langle\left.\omega\right|^{\text {even }}\left(\hat{b}_{p}^{\dagger}+f_{\omega}^{2} \cot \left(\frac{\pi p}{L}\right) \hat{b}_{-p}\right)=0\right.
$$

which follows directly from eq. (5.5).

Since $H=\sum_{p>0} H_{p},\left\langle\left.\omega\right|^{\text {even }} H=0\right.$ and $\left\langle\left.\omega\right|^{\text {even }}\right.$ is a product state also in momentum space, we have $\left\langle\left.\omega\right|^{\text {even }} H_{p}=0\right.$ separately. Therefore we can write

$$
\begin{aligned}
\sum_{x}\left\langle\left.\omega\right|^{\text {even }} \tilde{n}_{x} \exp (-H t) \mid \rho_{0}\right\rangle^{\text {even }} & =2 \sum_{p>0} f_{\omega}^{2} \cot \left(\frac{\pi p}{L}\right)\left\langle\left.\omega\right|^{\text {even }} \hat{b}_{p} \hat{b}_{-p} \exp (-H t) \mid \rho_{0}\right\rangle^{\text {even }} \\
& =2 \sum_{p>0} f_{\omega}^{2} \cot \left(\frac{\pi p}{L}\right)\left\langle\left.\omega\right|^{\text {even }} \sigma_{p}^{+} \exp \left(-H_{p} t\right) \mid \rho_{0}\right\rangle^{\text {even }}
\end{aligned}
$$


with $H_{p}=2\left(1-h \cos \left(\frac{\pi p}{L}\right)\right)-\left(\alpha_{p} \sigma_{p}^{x}+\beta_{p} \sigma_{p}^{y}+\gamma_{p} \sigma_{p}^{z}\right)$. The constants $\alpha_{p}, \beta_{p}$ and $\gamma_{p}$ are given by $\alpha_{p}=\left(\eta_{1}+\eta_{2}\right) \sin \left(\frac{\pi p}{L}\right), \beta_{p}=\mathrm{i}\left(\eta_{1}-\eta_{2}\right) \sin \left(\frac{\pi p}{L}\right)$ and $\gamma_{p}=\left(2 h-2 \cos \left(\frac{\pi p}{L}\right)\right)$. If we define a vector $\boldsymbol{a}$ by the components $\left(\alpha_{p}, \beta_{p}, \gamma_{p}\right)$ we have

$$
\exp \left(\boldsymbol{a} \cdot \boldsymbol{\sigma}_{\boldsymbol{p}} t\right)=\cosh (|\boldsymbol{a}| t)+\frac{\boldsymbol{a} \cdot \boldsymbol{\sigma}_{\boldsymbol{p}}}{|\boldsymbol{a}|} \sinh (|\boldsymbol{a}| t)
$$

where $\boldsymbol{\sigma}_{\boldsymbol{p}}=\left(\sigma_{p}^{x}, \sigma_{p}^{y}, \sigma_{p}^{z}\right)$ and $|\boldsymbol{a}|=\sqrt{\alpha_{p}^{2}+\beta_{p}^{2}+\gamma_{p}^{2}}$. Substituting the definitions above and using the identity $h^{2}+\eta_{1} \eta_{2}=1$ one finds that $|\boldsymbol{a}|=2-2 h \cos \left(\frac{2 \pi p}{L}\right)$. Rexpressing $\sigma_{p}^{x}$ and $\sigma_{p}^{y}$ in terms of $\sigma_{p}^{+}$, $\sigma_{p}^{-}$, one gets

$$
\begin{aligned}
\exp \left(\boldsymbol{a} \cdot \boldsymbol{\sigma}_{\boldsymbol{p}} t\right)= & \cosh \left(\left(2-2 h \cos \left(\frac{2 \pi p}{L}\right)\right) t\right)+\sinh \left(\left(2-2 h \cos \left(\frac{2 \pi p}{L}\right)\right) t\right) \times \\
& \times\left(\frac{h-\cos \left(\frac{2 \pi p}{L}\right)}{1-h \cos \left(\frac{2 \pi p}{L}\right)} \sigma_{p}^{z}+\frac{\eta_{2} \sin \left(\frac{2 \pi p}{L}\right)}{1-h \cos \left(\frac{2 \pi p}{L}\right)} \sigma_{p}^{-}+\frac{\eta_{1} \sin \left(\frac{2 \pi p}{L}\right)}{1-h \cos \left(\frac{2 \pi p}{L}\right)} \sigma_{p}^{+}\right)
\end{aligned}
$$

Developing $\exp \left(-H_{p} t\right)$ in (5.9) with the help of (5.11), using the algebra of the $\sigma$ matrices and the property of the $\left|\rho_{0}\right\rangle^{\text {even }}$ state

$$
\sigma_{p}^{-}\left|\rho_{0}\right\rangle^{\text {even }}=\left(\mu^{-2} \tan \left(\frac{\pi p}{L}\right)-\mu^{-4} \tan ^{2}\left(\frac{\pi p}{L}\right) \sigma_{p}^{+}\right)\left|\rho_{0}\right\rangle^{\text {even }}
$$

one gets after a little algebra the expression

$$
\begin{aligned}
\sum_{x}\left\langle\left.\omega\right|^{\text {even }} \tilde{n}_{x} \exp (-H t) \mid \rho_{0}\right\rangle^{\text {even }}=2 & \sum_{p>0} f_{\omega}^{2} \cot \left(\frac{\pi p}{L}\right)\left\langle\left.\omega\right|^{\text {even }} \sigma_{p}^{+} \mid \rho_{0}\right\rangle^{\text {even }} \\
\times & {\left[1+\left(\mu^{-2} \eta_{2}-(1+h)\right) \frac{1-e^{-4\left(1-h \cos \left(\frac{2 \pi p}{L}\right)\right) t}}{1-h \cos \left(\frac{2 \pi p}{L}\right)} \sin ^{2}\left(\frac{\pi p}{L}\right)\right] . }
\end{aligned}
$$

Now it can be easily shown that $\left\langle\left.\omega\right|^{e v e n} \sigma_{p}^{+} \mid \rho_{0}\right\rangle^{e v e n}=\frac{\mu^{2} \cot \left(\frac{\pi p}{L}\right)}{1+\mu^{2} f_{\omega}^{2} \cot ^{2}\left(\frac{\pi p}{L}\right)}$. Substituting this expression in $(5.13)$ we get

$$
\begin{aligned}
& \sum_{x}\left\langle\left.\omega\right|^{e v e n} \tilde{n}_{x} \exp (-H t) \mid \rho_{0}\right\rangle^{e v e n}=2 \sum_{p>0} \frac{\mu^{2} f_{\omega}^{2} \cot ^{2}\left(\frac{\pi p}{L}\right)}{1+\mu^{2} f_{\omega}^{2} \cot ^{2}\left(\frac{\pi p}{L}\right)} \\
& \times\left[1+\left(\mu^{-2} \eta_{2}-(1+h)\right) \frac{1-e^{-4\left(1-h \cos \left(\frac{2 \pi p}{L}\right)\right) t}}{1-h \cos \left(\frac{2 \pi p}{L}\right)} \sin ^{2}\left(\frac{\pi p}{L}\right)\right] .
\end{aligned}
$$

As stated above the values of $f_{\omega}$ and of $\mu$ can be found from the application of the transformation matrix to the original states. However, one can extract $f_{\omega}^{2}$ from the condition $\left\langle\left.\omega\right|^{e v e n} H_{p}=0\right.$. We obtain $f_{\omega}^{2}=\frac{1-h}{\eta_{2}}$, this result being valid for all the four cases of section 3. Separating the time-independent 
part of (5.14) and using this condition, one obtains after dividing by $L$ and taking the thermodynamic limit

$$
\begin{aligned}
\rho(t)= & \frac{1}{2 \pi} \int_{-\pi}^{\pi} d p \frac{1}{1+\frac{1+h}{1-h} \tan ^{2}\left(\frac{p}{2}\right)} \\
& -\frac{1}{2 \pi} \int_{-\pi}^{\pi} d p \frac{f_{\omega}^{2}\left(\eta_{2}-(1+h) \mu^{2}\right)}{\mu^{2} f_{\omega}^{2}+\tan ^{2}\left(\frac{p}{2}\right)} \frac{e^{-4(1-h \cos p) t}}{1-h \cos p} \sin ^{2}\left(\frac{p}{2}\right) .
\end{aligned}
$$

Performing the first integral, one obtains

$$
\rho(t)=\frac{1}{1+\sqrt{\frac{1+h}{1-h}}}-\frac{1}{2 \pi} \int_{-\pi}^{\pi} d p \frac{f_{\omega}^{2}\left(\eta_{2}-(1+h) \mu^{2}\right)}{\mu^{2} f_{\omega}^{2}+\tan ^{2}\left(\frac{p}{2}\right)} \frac{e^{-4(1-h \cos p) t}}{1-h \cos p} \sin ^{2}\left(\frac{p}{2}\right)
$$

which is the central result of this section.

When $h= \pm 1$, the generic long-time behaviour of the density is $\rho(t) \simeq \rho_{\infty}+\mathcal{A} t^{-1 / 2}$, where $\mathcal{A}$ is some constant. On the other hand, when $|h|<1$, the generic long time behaviour is $\rho(t) \simeq \rho_{\infty}+$ $\mathcal{A}^{\prime} t^{-3 / 2} \exp (-t / \tau)$, where the relaxation time is

$$
\tau^{-1}=4(1-h)
$$

and $\mathcal{A}^{\prime}$ is another constant. To proceed further we need the values of $h, \eta_{2}, \mu^{2}$ and $f_{\omega}$ for each of the four cases of table 3. We have

1. For model I, we use eqns. (3.58, 3.59) together with eq. (5.3) to express the action of the Pauli spin matrices in the state $\langle\omega|$ in terms of $\tilde{n}_{x}$. We obtain for the particle density

$$
\tilde{\rho}(t)=\frac{2}{\sqrt{1+A^{2}}} \rho(t)-\frac{1}{\sqrt{1+A^{2}}}
$$

where $\rho(t)$ is given by (5.16) with $h$ and $\eta_{2}$ as a function of $A$ were defined in section 3 and

$$
f_{\omega}^{2}=-\left[\frac{\sqrt{1+A^{2}}+1}{A}\right]^{2}, \mu^{2}=-\frac{A^{2}}{\left(1+\sqrt{1+A^{2}}\right)^{2}}\left[\frac{1+\tilde{\rho_{0}} \sqrt{1+A^{2}}}{1-\tilde{\rho_{0}} \sqrt{1+A^{2}}}\right]^{2}
$$

where $\tilde{\rho}_{0}$ is the initial particle density. Using (5.16) and (5.18), we get the steady state particle density

$$
\tilde{\rho}_{\infty}=\frac{1}{1+A^{2}} .
$$

Model I describes particles undergoing diffusion and interacting with each other through death and creation processes, as defined in table 11. Two extreme limits can immediately be understood intuitively. When the death processes are absent $(h \rightarrow-1$ or $A \rightarrow 0)$, the steady state density is 
$\rho_{\infty}=1$ and the approach to the steady state is algebraic. On the other hand, if only the death process is present $(h \rightarrow 0$ or $A \rightarrow \infty), \rho_{\infty}=0$ and the approach to the steady state is exponential. While in the first case, particles must diffuse to create local configurations which are favorable for more reactions taking place and which involves time scales of order $t^{1 / 2}$, in the second case diffusion is absent and particles are just taken out of the system with a certain rate. Thus a finite cluster of particles will disappear within a finite time which is proportional to the cluster size and the death rate.

2. For model II, where we have symmetric diffusion with coagulation and decoagulation, we repeat what we have done for the case above. This model had already been studied in detail before 38, 63. We obtain for the density

$$
\tilde{\rho}(t)=\frac{2}{\sqrt{1+A^{2}}} \rho(t)-\frac{1}{\sqrt{1+A^{2}}}+1
$$

with $\rho(t)$ given as above but with $h$ and $\eta_{2}$ given by (3.48) and

$$
f_{\omega}^{2}=-\left[\frac{A}{\sqrt{1+A^{2}}+1}\right]^{2}, \quad \mu^{2}=-\left[\frac{\sqrt{1+A^{2}}+1}{A}\right]^{2}\left[\frac{1-\left(1-\widetilde{\rho}_{0}\right) \sqrt{1+A^{2}}}{1+\left(1-\widetilde{\rho}_{0}\right) \sqrt{1+A^{2}}}\right]^{2} .
$$

The steady state density is

$$
\tilde{\rho}_{\infty}=1-\frac{1}{1+A^{2}}
$$

We point out that under a particle-hole transformation $\widetilde{\rho} \rightarrow 1-\widetilde{\rho}$ (and consequently $\mu \rightarrow 1 / \mu$ and $\left.f_{\omega}^{2} \rightarrow 1 / f_{\omega}^{2}\right)$ we just recover the results found for model I.

3. For model III, where we have symmetric diffusion with decoagulation and death, we repeat the steps of the previous cases. We obtain for the density

$$
\tilde{\rho}(t)=-\frac{2 A}{A^{2}-1} \rho(t)+\frac{1}{1+A}
$$

with $h$ and $\eta_{2}$ given by $(3.45)$ and

$$
f_{\omega}^{2}=-\left[\frac{A-1}{A+1}\right]^{2}, \mu^{2}=-\left(\frac{A \tilde{\rho}_{0}-\left(1-\tilde{\rho}_{0}\right)}{A \tilde{\rho}_{0}+\left(1-\tilde{\rho}_{0}\right)}\right)^{2}
$$

In order to calculate the equilibrium density we have to distinguish three different cases, i.e. $|A|<1,|A|=1$ and $|A|>1$. In the first case the steady state density is $\rho_{\infty}=1$, this result being easy to understand since the rate of decoagulation $(\widetilde{\beta}=2)$ is larger than the death rate $\left(\widetilde{\delta}=2 A^{2}\right)$. On the other hand, when $|A|>1, \rho_{\infty}=0$. When $|A|=1$ the two processes 
equilibrate each other and $\rho_{\infty}=1 / 2$. From (3.45) we see that when $A= \pm 1, h= \pm 1$ and the decay towards the steady state is algebraic. For all other values of $A$ the decay is exponential.

This is in agreement with numerical studies in the biased voter model, see 62 and references therein. The exponential decay of the biased voter model can be intuitively understood using the same argument as presented for model I. For the symmetric voter model, the algebraic decay is expected since this model is dual [64 to the Glauber-Ising model [14 at zero temperature.

4. Finally, model IV just corresponds to model of symmetric diffusion with pair creation and annihilation. We simply have $\tilde{\rho}(t)=\rho(t)$ with $\rho(t)$ given by (5.16), with $h=\tilde{\alpha}-\tilde{\nu}$ and $\eta_{1}=2 \tilde{\alpha}$, $\eta_{2}=2 \tilde{\nu}, 2 \tilde{\alpha}$ and $2 \tilde{\nu}=2-2 \tilde{\alpha}$ being respectively the rates of for pair annihilation and pair creation, see table 1. The constant $f_{\omega}=1$ and $\mu=\tilde{\rho}_{0} /\left(1-\tilde{\rho}_{0}\right)$.

If we take $h= \pm 1$, then we have pure pair annihilation and pure pair creation, respectively, and we recover previously known results [54, 55, 38]. The decay to the steady state of the particle density is algebraic and of the form $t^{-1 / 2}$.

For $0<h<1$, we obtain an unexpected result. For $\mu=0$, the time-dependent density had been calculated before [61] and we reproduce their result. The long-time behaviour of the density is generically $\rho(t)-\rho_{\infty} \sim t^{-1 / 2} \exp (-t / \tau)$. On the other hand, if $0<\mu \leq 1$, we get from (5.16) that $\rho(t)-\rho_{\infty} \sim t^{-3 / 2} \exp (-t / \tau)$. In addition, we have because of the particle-hole symmetry that the result is invariant under the transformation $h \rightarrow-h, \mu \rightarrow 1 / \mu$. So the long-time behaviour of the system does depend in this case on the initial condition.四

In summary, the main result of this section is eq. (5.16), together with eqs. (5.18, 5.21, 5.24) which yield the particle densities in models I, II and III. While we have limited ourselves to extract but the leading behaviour for large times, further terms could be readily obtained.

\section{Conclusions}

In this paper, we have addressed the problem of explicitly calculating the long-time behaviour of timedependent averages of several types of reaction-diffusion systems in situations (here in low dimensions) where the presence of strong fluctuation effects prevents the use of simple kinetic equations. We have seen that, in particular in one dimension, the relationship of the master equation with the quantum Hamiltonians of known integrable quantum chains can be fruitfully employed. Our aim has been to

\footnotetext{
${ }^{15}$ This comment also applies to models I, II and III if $\mu=0$ and $0<h<1$ or $\mu=\infty$ and $-1<h<0$.
} 
show how to obtain, through a certain similarity transformation of the form eq. (2.19), the equivalent stochastic systems as characterized through their quantum Hamiltonian. Technically, the difficulty lies in satisfying simultaneously the set of coupled inequalities coming from the positivity of the rates which enter into the master equation, but from the examples presented here it should be clear how to proceed for more general models.

We have obtained the following:

1. Starting from the most general free fermion Hamiltonian with site-independent nearest-neighbor interactions, eq. (3.65), we have found all equivalent (non-trivial) stochastic systems, as listed in table 3. The temporal behaviour of the averages of all systems can be found from a single calculation as shown in section 5 and we have seen that a single parameter $h$ describes the physics. Specifically, we have for the density $\rho(t)$ at large times

$$
\rho(t)-\rho_{\infty} \sim\left\{\begin{array}{llr}
t^{-1 / 2} & ; \quad & h=1 \\
t^{-3 / 2} \exp (-t / \tau) & ; \quad 0<h<1
\end{array}\right.
$$

(provided the initial density $\rho_{0} \neq 0$ ) where $\rho_{\infty}$ is the steady-state density and $\tau=\frac{1}{4}(1-h)^{-1}$ is the relaxation time. Because of the particle-hole symmetry, the same form holds for the density of holes when $-1 \leq h<0$. It is known that there exist larger classes of reaction-diffusion systems which have the same Hamiltonian spectrum as a free fermion chain with site-dependent interactions [54, 48]. The explicit transformation generalising (2.19) between these systems remains to be found. Another interesting extension would be the consideration of these stochastic systems on quasiperiodically modulated lattices.

2. As a second example, we have started from the most general Hamiltonian which has the same spectrum as the XXZ chain (with site-independent interactions) and is thus treatable thorugh the Bethe ansatz. Up to a rescaling in the rates, we have seen in section 4 that there is a threeparameter family of stochastic systems equivalent to the integrable XXZ chain. The conditions for this type of integrability can be stated exclusively in terms of the reaction rates. Furthermore, we have seen that if we require parity symmetry (no left-right bias in the reaction rates) this system is equivalent (working with the Hamiltonian $H^{I I I}$ in section 4) to the known reaction-diffusion system eq. (2.21) with only irreversible reactions. The long-time behaviour of the model (2.21) depends on the two parameters $\delta$ and $\Delta=1+\delta-\alpha-\gamma$ and is given in table 2. While for $\delta=0$, densities and correlators decay algebraically in time, we find that for $\delta \neq 0$, the relaxation times 
for multiparticle correlators depend on wether or not there exist low-lying bound states in the multiparticle sectors of the XXZ chain.

This observation should remind us to be careful when extrapolating results an insights from many ongoing calculations, which often implicitly contain a free fermion condition (which is usually formulated as specifying an infinite reaction rate on the encounter of two particles), to more general situations, where non-trivial bound states of the quantum chain may reflect themselves in the values of the relaxation times.

\section{Acknowledgements}

We are grateful to G.M. Schütz for valuable comments during the early stages of this work. It is also a pleasure to thank R.B. Stinchcombe and R. Wilman for useful discussions. MH and EO were supported by a grant of the EC program 'Human Capital and Mobility'. JES is supported by the grant PRAXIS XXI/BD/3733/94 - Portugal. 


\section{Appendix A. Non-hermitian free fermion Hamiltonians}

Here we discuss how the standard techniques [67] of reducing the diagonalization of $2^{L} \times 2^{L}$ free fermion Hamiltonian matrices to the diagonalization of a much smaller matrix can be extended to non-hermitian cases as well. For periodic boundary conditions, the complete diagonalization can be achieved by a sequence of Jordan-Wigner, Fourier and Bogoliubov transformations, as detailed in [55, 61, 63. Here we want a general formulation which treats all boundary conditions and space-dependent couplings on the same level.

The problem of diagonalizing a non-hermitian quantum Hamiltonian such as (3.1) is through a Jordan-Wigner transformation

$$
\sigma_{m}^{+}=c_{m} \exp \left(i \pi \sum_{j=1}^{m-1} c_{j}^{\dagger} c_{j}\right) \quad, \quad \sigma_{m}^{-}=\exp \left(i \pi \sum_{j=1}^{m-1} c_{j}^{\dagger} c_{j}\right) c_{m}^{\dagger}
$$

reduced to diagonalizing the following quadratic form

$$
H=-\sum_{n, m=1}^{L}\left[c_{n}^{\dagger} A_{n m} c_{m}+\frac{1}{2} c_{n}^{\dagger} B_{n m} c_{m}^{\dagger}+\frac{1}{2} c_{n} D_{n m} c_{m}\right]
$$

where $A, B, D$ are $L \times L$ matrices. For the system (3.1) one has for periodic boundary conditions

$$
\begin{aligned}
& A=\left(\begin{array}{cccccc}
h & D_{2} & & & & -D_{1}(-1)^{\mathcal{N}} \\
D_{1} & h & D_{2} & & & \\
& D_{1} & h & D_{2} & & \\
& & \ddots & \ddots & \ddots & \\
& & & D_{1} & h & D_{2} \\
-D_{2}(-1)^{\mathcal{N}} & & & & D_{1} & h
\end{array}\right)
\end{aligned}
$$

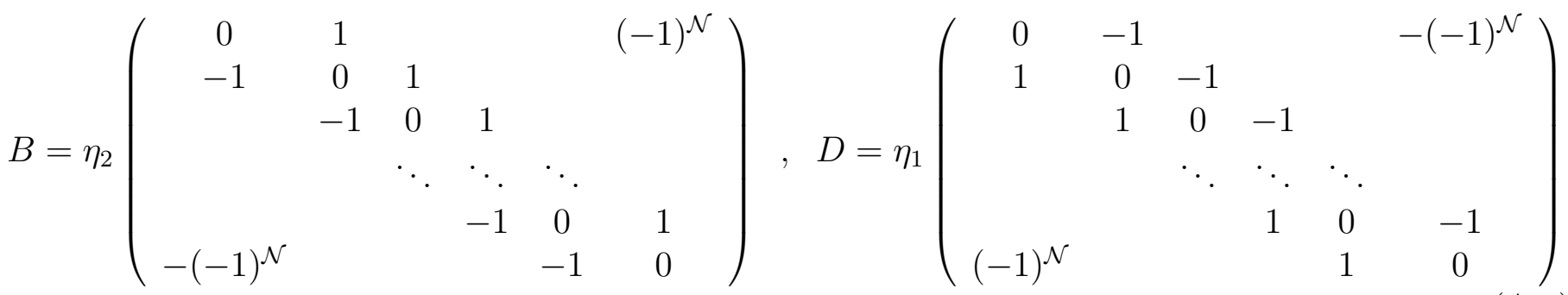

where $\mathcal{N}=\sum_{j=1}^{L} c_{m}^{\dagger} c_{m}$ is the fermion number operator and the $c_{m}$ satisfy the usual anticommutation relations. For more general nearest-neighbor interactions and/or boundary conditions, the precise form of the matrices $A, B, D$ will be affected but not the diagonalization technique to be described.

Following [67, we seek new fermionic variables $\xi_{q}$ which render $H$ diagonal

$$
H=\sum_{q} \Lambda_{q}\left(\xi_{q}^{+} \xi_{q}-\frac{1}{2}\right)
$$


and which are defined through the following canonical transformation

$$
\xi_{q}^{+}=\sum_{m}\left(\alpha v_{q m} c_{m}^{\dagger}+\alpha^{-1} u_{q m} c_{m}\right) \quad, \quad \xi_{q}=\sum_{m}\left(\alpha u_{q m}^{*} c_{m}^{\dagger}+\alpha^{-1} v_{q m}^{*} c_{m}\right)
$$

where $u, v$ are $L \times L$ matrices and $\alpha$ is a free parameter. For $\alpha=1$ we recover the canonical transformation for hermitian $H$ [67]. We stress that $\xi_{q}^{+} \neq \xi_{q}^{\dagger}$, the hermitian conjugate of $\xi_{q}$, when $\alpha \neq 1$. But all what we need are the anticommutation relations

$$
\left\{\xi_{q}^{+}, \xi_{p}\right\}=\delta_{q, p},\left\{\xi_{q}^{+}, \xi_{p}^{+}\right\}=\left\{\xi_{q}, \xi_{p}\right\}=0
$$

which imply that

$$
\sum_{m}\left(u_{q m} u_{p m}^{*}+v_{q m} v_{p m}^{*}\right)=\delta_{q, p}, \sum_{m}\left(u_{q m} v_{p m}+v_{q m} u_{p m}\right)=0
$$

We can thus use $\xi_{q}^{+}$and $\xi_{q}$ as particle creation and annihilation operators to generate the entire spectrum of $H$ from the one-particle energies $\Lambda_{q}$. Now, from (A.5) we must have $\left[\xi_{q}, H\right]=\Lambda_{q} \xi_{q}$ and this leads to

$$
\Lambda_{q} \alpha u_{q m}^{*}=\sum_{n}\left(\alpha u_{q n} A_{m n}-\alpha^{-1} v_{q n} B_{n m}\right), \Lambda_{q} \alpha^{-1} v_{q m}^{*}=-\sum_{n}\left(\alpha u_{q n} D_{n m}+\alpha^{-1} v_{q n} A_{n m}\right)
$$

From the structure of the Hamiltonian (3.1), it is apparent that $B=-\left(\eta_{2} / \eta_{1}\right) D$. We now choose $\alpha=\left(\eta_{2} / \eta_{1}\right)^{1 / 4}$. Introducing a vector notation $\left(\overleftarrow{u_{q}}\right)_{n}=u_{q n}$, we then have

$$
\Lambda_{q}\left({\overleftarrow{u_{q}}}^{*},{\overleftarrow{v_{q}}}^{*}\right)=\left({\overleftarrow{u_{q}}}^{*}, \overleftarrow{v}_{q}^{*}\right)\left(\begin{array}{cc}
A^{T} & -\alpha^{2} D \\
\alpha^{2} D & -A
\end{array}\right)
$$

which reduces the problem to the diagonalization of a $2 L \times 2 L$ matrix.

A further simplification can be achieved when $A$ is symmetric, $A=A^{T}$ (which in the context of the model (3.1) means $\left.D_{1}=D_{2}\right)$. We then find, analogously to 67

$$
\Lambda_{q}^{2}\left(\overleftarrow{u}_{q}^{*}+\overleftarrow{v}_{q}^{*}\right)=\left(\overleftarrow{u}_{q}^{*}+\overleftarrow{v}_{q}^{*}\right) \mathcal{M}, \mathcal{M}=\left(A+\alpha^{2} D\right)\left(A-\alpha^{2} D\right)
$$

and have reduced the problem to the diagonalization of an $L \times L$ matrix. In particular, since $\mathcal{M}$ is hermitian, it follows in this case that all $\Lambda_{q}$ are real and can be taken to be positive. The hermitian case treated in [67] is recovered for $\alpha=1$.

\section{Appendix B. Some remarks on the transformation (3.3)}

We add some technical remarks on the transformation (3.3) used to find stochastic systems from the free fermion Hamiltonian (3.1). 


\section{B.1 The case $D_{1}+D_{2}=0$}

When $D_{1}+D_{2}=0$, the analysis of section 3 has to be repeated. First, probability conservation (2.8) is implemented by fixing $\eta_{1,2}$

$$
\eta_{1,2}= \pm h(\eta(a, Y))^{ \pm 1} \quad, \quad \eta(a, Y)=\left(\frac{1-a+Y+a Y}{-1+a+Y+a Y}\right)^{2}
$$

The parity symmetric matrix elements are

$$
\begin{aligned}
-2 \widetilde{\alpha} & =-a^{2} h\left(1-a+Y^{2}+a Y^{2}\right) \cdot G_{1} \cdot M \\
-2 \widetilde{\nu} & =a^{-1} h\left(-1+a+Y^{2}+a Y^{2}\right) \cdot G_{2} \cdot M \\
-\widetilde{\gamma} & =\frac{1}{2} h a\left(a^{2}-1\right)\left(Y^{2}-1\right) \cdot G_{1} \cdot M \\
-\widetilde{\delta} & =h a\left(Y^{2}-1\right)\left(1-a+Y^{2}+a Y^{2}\right) \cdot G_{3} \cdot M \\
-\widetilde{\beta} & =-h\left(Y^{2}-1\right)\left(-1+a+Y^{2}+a Y^{2}\right) \cdot G_{3} \cdot M \\
-\widetilde{\sigma} & =\frac{1}{2 a} h\left(a^{2}-1\right)\left(Y^{2}-1\right) \cdot G_{2} \cdot M \\
-\widetilde{D} & =-\frac{1}{2} h\left(a^{2}-1\right)\left(Y^{2}-1\right)^{2} \cdot G_{2} \cdot M
\end{aligned}
$$

where

$$
\begin{aligned}
G_{1} & =1-2 a+a^{2}+6 Y^{2}-2 a^{2} Y^{2}+Y^{4}+2 a Y^{4}+a^{2} Y^{4} \\
G_{2} & =1-2 a+a^{2}-2 Y^{2}+6 a^{2} Y^{2}+Y^{4}+2 a Y^{4}+a^{2} Y^{4} \\
G_{3} & =1-2 a+a^{2}+Y^{2}+2 a Y^{2}+a^{2} Y^{2} \\
M^{-1} & =Y(1-a+Y+a Y)^{2}(-1+a+Y+a Y)^{2}
\end{aligned}
$$

and we see that $h$ only enters into the matrix elements as a constant factor and thus plays no role whatsoever. The parity nonsymmetric matrix elements are

$$
\begin{aligned}
\widetilde{\delta}^{\prime} & =a\left(D_{1}-g\right) \frac{Y^{2}-1}{4 Y} \\
\widetilde{\sigma}^{\prime} & =a^{-1}\left(D_{1}+g\right) \frac{Y^{2}-1}{4 Y} \\
\widetilde{\gamma}^{\prime} & =a\left(D_{1}+g\right) \frac{Y^{2}-1}{4 Y} \\
-\widetilde{\beta}^{\prime} & =a^{-1}\left(D_{1}-g\right) \frac{Y^{2}-1}{4 Y} \\
\widetilde{D^{\prime}} & =-D_{1} \frac{Y^{2}+1}{2 Y}
\end{aligned}
$$


Second, we apply the reality criterion. Since both $\widetilde{\delta}^{\prime} / \widetilde{\beta}^{\prime}$ and $\widetilde{\gamma}^{\prime} / \widetilde{\sigma}^{\prime}$ must be real, it follows that $a^{2}$ is real. In fact, we find that the reality conditions (3.21) also hold here. Third, one localizes the curves in $(a, Y)$ space where the parity symmetric rates change sign. Mapping out the corresponding areas, we find as before that the positivity conditions (2.8) for the rates cannot simultaneously be satisfied. Finally, one might try to use the factorized structure of the matrix elements in (B.2) to put selectively some rates to zero. Carrying out the calculation, we find that no stochastic systems occur, since there will be always pairs of parity-symmetric rates which are not positive multiples of each other.

In conclusion, the case $D_{1}+D_{2}=0$ does not lead to any stochastic system.

\section{B.2 Alternative forms of the transformation matrix}

Here we briefly discuss a few alternative transformation matrices. Begin with the transformation matrix

$$
B=\left(\begin{array}{cc}
x & y \\
0 & x^{-1}
\end{array}\right)
$$

where $x$ and $y$ are free parameters. It is enough here to consider the unbiased case $D_{1}=D_{2}=1$. Probability conservation is then implemented through $g=0$ and

$$
\eta_{1}=(h+1)(1+x y)^{2} / x^{4}, \quad \eta_{2}=(1-h) x^{4} /(1+x y)^{2}
$$

For the rates, we find in particular

$$
\widetilde{\sigma}=-\widetilde{\beta}=(h-1) x y /(1+x y)^{2}
$$

and, from the positivity conditions, we must have either $x y=0$ or $h=1$. In the first case, the transformation $B$ is singular for $x=0$ and trivial for $y=0$. In the second case, $\eta_{2}=0$ and we find

$$
\widetilde{H}+1=\left(\begin{array}{cccc}
0 & 0 & 0 & -2(1+2 x y) \\
0 & 1 & -1 & 2 x y \\
0 & -1 & 1 & 2 x y \\
0 & 0 & 0 & 2
\end{array}\right)
$$

which is indeed stochastic for $-\frac{1}{2} \leq x y \leq 0$. We recover the annihilation-coagulation model [10, 39] discussed in section 2. As discussed in section 3, we can include surface fields to allow for biased reaction-diffusion rates. Alternatively, one might try

$$
B=\left(\begin{array}{cc}
x & -y^{-1} \\
y & 0
\end{array}\right)
$$

The only stochastic form is $(\mathbb{B . 8})$ with $y \rightarrow 1 / y$. Considering the inverses of the above transformation matrices corresponds to the particle-vacancy exchange $A \leftrightarrow \emptyset$. 
Finally, we consider (3.1) with $D_{1}=D_{2}=D$ and $\eta_{1}=\eta_{2}=\eta$. The transformation matrix is

$$
B=\left(\begin{array}{ll}
b_{11} & b_{12} \\
b_{21} & b_{22}
\end{array}\right)
$$

Let $\widetilde{C}_{m}=\sum_{k=1}^{4}\left(\widetilde{H}_{j, j+1}\right)_{k, m}$. A necessary condition for probability conservation is $\widetilde{C}_{2}=\widetilde{C}_{3}$. This implies

$$
\frac{\left(b_{11}+b_{21}\right)\left(b_{21}+b_{22}\right) 2 g}{\operatorname{det} B}=0
$$

The case $g=0$ was discussed in section 3 . If $b_{11}+b_{21}=0$, the two other conditions from probability conservation require that

$$
h-\eta\left(\frac{b_{22}}{b_{11}}\right)^{2}=h-\eta\left(\frac{b_{12}}{b_{11}}\right)^{2}=h+\eta \frac{b_{12} b_{22}}{b_{11}^{2}}
$$

This implies that either $b_{12}^{2}=b_{22}^{2}$ or that $\eta=0$. In the first case, $b_{12}=-b_{22}$ renders $B$ singular and from $b_{12}=b_{22}$, it follows $\eta=0$ anyway. So we take the second case. From $\widetilde{\alpha}$ and $\widetilde{\gamma}$, it then follows that $D=0$ as well. The resulting Hamiltonian is

$$
\widetilde{H}=\left(\begin{array}{cccc}
-2 h V & 2 h_{2} U & 2 h_{1} U & 0 \\
2 h_{2} V & -2 h_{1} V-2 h_{2} U & 0 & 2 h_{1} U \\
2 h_{1} V & 0 & -2 h_{1} U-2 h_{2} V & 2 h_{2} U \\
0 & 2 h_{1} V & 2 h_{2} V & -2 h U
\end{array}\right)
$$

where

$$
U=\frac{b_{12}}{b_{12}+b_{22}}, \quad V=\frac{b_{22}}{b_{12}+b_{22}}
$$

This is stochastic provided $h_{1}, h_{2} \leq 0$ and $b_{12} \cdot b_{22} \geq 0$. Physically, the system is trivial, since it consists of a set of non-interacting particles which are spontaneously created and decay spontaneously. Similarly, if from (B.11) we have $b_{21}+b_{22}=0$, we get $\eta=D=0$ and

$$
\widetilde{H}=-\left(\begin{array}{cccc}
-2 h V & 2 h_{2} U & 2 h_{1} U & 0 \\
2 h_{2} V & -2 h_{1} V-2 h_{2} U & 0 & 2 h_{1} U \\
2 h_{1} V & 0 & -2 h_{1} U-2 h_{2} V & 2 h_{2} U \\
0 & 2 h_{1} V & 2 h_{2} V & -2 h U
\end{array}\right)
$$

with

$$
U=\frac{b_{11}}{b_{11}+b_{21}}, \quad V=\frac{b_{21}}{b_{11}+b_{21}}
$$

which is stochastic if $h_{1}, h_{2} \geq 0$ and $b_{11}$ and $b_{21}$ have the same sign. The physics is again trivial.

\section{Appendix C. Quantum chain formulation for the biased voter model}

Conventionally, the biased voter model [62] is defined as a Markov process where the sites on the lattice are either in state 0 or state 1 . If site $j$ is in state 0 , it changes to state 1 at a rate $\lambda r_{1}$ where $r_{1}$ is the 
number of nearest neighbors of the site $j$ which are in state 1 . Similarly, sites in state 1 change to state 0 at rate $r_{0}$. Thus the elementary processes involve three sites rather than two as it is in the quantum Hamiltonians we consider here. Now consider the stochastic quantum Hamiltonian $H=\sum_{j} H_{j, j+1}$ with two-body matrix

$$
H_{j, j+1}=\left(\begin{array}{cccc}
0 & -1 & -1 & 0 \\
0 & \lambda+1 & 0 & 0 \\
0 & 0 & \lambda+1 & 0 \\
0 & -\lambda & -\lambda & 0
\end{array}\right)
$$

From this we construct the three-sites contribution

$$
H_{j-1, j, j+1}=\mathbf{1} \otimes H_{j, j+1}+H_{j-1, j} \otimes \mathbf{1}
$$

which completely describes the transitions of the central site $j$. The transitions of the central site occur with the rates

$$
\begin{array}{cccccc}
\emptyset A \emptyset \rightarrow \emptyset \emptyset \emptyset & 2 & ; & A \emptyset A & \rightarrow A A A & 2 \lambda \\
\emptyset A A \rightarrow \emptyset \emptyset A & 1 & ; & \emptyset \emptyset A & \rightarrow \emptyset A A & \lambda \\
A A \emptyset \rightarrow A \emptyset \emptyset & 1 & ; & A \emptyset \emptyset \rightarrow A A \emptyset & \lambda \\
A A A \rightarrow A \emptyset A & 0 & ; & \emptyset \emptyset \emptyset \rightarrow \emptyset A \emptyset & 0
\end{array}
$$

Now, if one identifies $A$ with the state 1 and $\emptyset$ with the state 0 , one reproduces for the central site $j$ the rules of the biased voter model as specified above. Carrying out the sum over all lattice sites yields the same rules for all sites $j$.

\section{Appendix D. The case $P=0$ for the $\mathrm{XXZ}$ chain}

We give here the details of the calculations leading to the results stated in section 4 . The cases II and III must be considered separately.

\section{D.1 Case II}

The following notation is used. We write $b_{1}=b_{11}$ and $b_{2}=b_{22}$ and use the parameters $Q$ and $\Omega$ from table 4 . Then the parity-symmetric rates are

$$
\begin{aligned}
\widetilde{\alpha} & =\frac{\Omega}{2} \frac{b_{1}^{3}}{b_{2}} \\
\widetilde{\beta} & =\frac{b_{1}-1}{2 b_{2}}\left[2 \Omega b_{1}\left(b_{1}-1\right)-b_{2} Q\right] \\
\widetilde{\gamma} & =-\frac{b_{1}}{2 b_{2}}\left[2 \Omega b_{1}\left(b_{1}-\frac{1}{2}\right)-b_{2} Q\right] \\
\widetilde{\delta} & =\frac{b_{1}}{2 b_{2}}\left[2 \Omega b_{1}\left(b_{1}-1\right)+b_{2} Q\right]
\end{aligned}
$$




$$
\begin{aligned}
\widetilde{\nu} & =\frac{\Omega}{2} \frac{\left(b_{1}-1\right)^{3}}{b_{2}} \\
\widetilde{\sigma} & =-\frac{b_{1}-1}{2 b_{2}}\left[2 \Omega\left(b_{1}-\frac{1}{2}\right)\left(b_{1}-1\right)+b_{2} Q\right] \\
\widetilde{D} & =-\frac{1}{2 b_{2}}\left[2 \Omega\left(b_{1}-\frac{1}{2}\right)\left(b_{1}-1\right)+b_{2}\left(h_{23}+h_{32}\right)\right]
\end{aligned}
$$

Now, we can always arrange to have $\Omega$ positive. To see this, recall that the transformation matrix $B$ viewed as function of $b_{2}$ satisfies

$$
B\left(b_{2}\right)=B\left(-b_{2}\right)\left(\begin{array}{cc}
1 & 0 \\
0 & -1
\end{array}\right)
$$

Therefore a change of sign in $b_{2}$ changes the sign of the matrix elements $h_{12}, h_{24}, h_{34}$. Since $h_{12}$ does not occur in eqs. (D.1), we can always absorb the sign of $\Omega$ into $b_{2}$. Next, from the positivity of $\widetilde{\alpha}$ and $\widetilde{\nu}$ one has the inequalities $\Omega b_{1} b_{2} \geq 0$ and $\Omega\left(b_{1}-1\right) b_{2} \geq 0$. The following two cases are to be distinguished: 1. $b_{1} \geq 1$ and $b_{2} \geq 0$. From $\widetilde{\beta} \geq 0$ we get $b_{1}\left(b_{1}-1\right) \geq Q /(2 \Omega) b_{2}$ or $b_{1} \geq 1+\left(Q /(2 \Omega)\left(b_{2} / b_{1}\right)=: 1+\mathcal{U}\right.$. On the other hand, from $\widetilde{\gamma} \geq 0$ we have $b_{1}\left(b_{1}-\frac{1}{2}\right) \leq Q /(2 \Omega) b_{2}$ or $1 \leq b_{1} \leq \frac{1}{2}+(Q / 2 \Omega)\left(b_{2} / b_{1}\right)=\frac{1}{2}+\mathcal{U}$.

This implies that $\mathcal{U} \geq \frac{1}{2}>0$. Now, taking the two inequalities together, we have $1+\mathcal{U} \leq b_{1} \leq \frac{1}{2}+\mathcal{U}$ which is impossible. The only escape is to set $\Omega=Q=0$.

2. $b_{1} \leq 0$ and $b_{2} \leq 0$. From the positivity of $\widetilde{\gamma}$ and $\tilde{\sigma}$ we get $2 \Omega\left(b_{1}-x\right)\left(b_{1}-1 / 2\right) \mp b_{2} Q \leq 0$, and $x=0,1$, respectively. Adding these two relations gives $\left(b_{1}-1\right)^{2} \leq 1 / 2$, which would imply $0 \geq b_{1}=1 / 2$, which is impossible. Again the only escape is to set $\Omega=Q=0$.

But if $\Omega=Q=0$, all rates with the exception of $\widetilde{D}$ vanish, which means that the only possibility is pure diffusion.

\section{D.2 Case III}

Using the same notation as above for case II, we find the parity-symmetric rates

$$
\begin{aligned}
-2 \widetilde{\alpha} & =b_{1}^{2}\left(-b_{1}(\Omega+Q)\right) /\left(b_{1}+b_{2}-1\right) \\
-2 \widetilde{\nu} & =\left(1-b_{1}\right)^{2}\left(-b_{1}(\Omega+Q)+\Omega+Q\right) \\
-2 \widetilde{\gamma} & =b_{1}\left(2(\Omega+Q) b_{1}^{2}-b_{1}(\Omega+2 Q)-b_{2} Q+Q\right) /\left(b_{1}+b_{2}-1\right) \\
-2 \widetilde{\delta} & =-b_{1}\left(2(\Omega+Q) b_{1}^{2}-b_{1}(2 \Omega+Q)+b_{2} Q-Q\right) /\left(b_{1}+b_{2}-1\right) \\
-2 \widetilde{\beta} & =\left(1-b_{1}\right)\left(2(\Omega+Q) b_{1}^{2}-b_{1}(2 \Omega+3 Q)-b_{2} Q+Q\right) /\left(b_{1}+b_{2}-1\right) \\
-2 \widetilde{\sigma} & =\left(b_{1}-1\right)\left(2(\Omega+Q) b_{1}^{2}-b_{1}(3 \Omega+2 Q)+b_{2} Q+\Omega\right) /\left(b_{1}+b_{2}-1\right) \\
-2 \widetilde{D} & =\left[2 b_{1}^{3}(\Omega+Q)-b_{1}^{2}(3 \Omega+3 Q)+b_{1}\left(\Omega+Q+h_{23}+h_{32}\right)\right. \\
& \left.+\left(b_{2}-1\right)\left(h_{23}+h_{32}\right)\right] /\left(b_{1}+b_{2}-1\right)
\end{aligned}
$$


For a single steady state we have $0<b_{1}<1$ and we get the inequalities $(Q+\Omega) b_{1} \leq 0$ from $\widetilde{\alpha} \geq 0$ and $(Q+\Omega)\left(b_{1}-1\right) \leq 0$ from $\widetilde{\nu} \geq 0$. This implies that $h_{44}=Q=-\Omega$. Inserting into the rates, one sees that the remaining positiviy conditions become

$$
\left(b_{1}+b_{2}-1\right)>0,\left(h_{23}+h_{32}\right) \leq 0
$$

and one recovers the stochastic Hamiltonian given in section 4.

\section{Appendix E. The long time behaviour of correlation functions}

In this appendix we give a brief discussion of the results presented in Table 2, namely we show how the long time behaviour of the one and two-point correlation functions may be obtained by a truncation of the equations of motion for these quantities which becomes exact at long times [10. It is easy to see that the long time behaviour of the one and two-point equal time correlation functions is determined by the lowest lying state in the one and two-particle sectors respectively, which is non-orthogonal to the initial state, provided that there is not a state in a sector with a larger number of particles that has a lower energy. We have seen in section 2 that the spectrum of Hamiltonian (2.21) is identical to the spectrum of $H_{X X Z}(h, \Delta, \delta)$. So the study of the spectrum of the one and two-particle sector of $H_{X X Z}$ allows one to obtain directly the time decay of these two correlation functions. In particular it can be shown, and we will do this below, that there is a line of bounds states in the two particle sector for any $\Delta \neq 0$. The presence of those states has to be taken into account in the discussion of the behaviour of the two point correlation functions. The energy of lowest state in the one-particle sector is, as given by (4.5), equal to $E=2 \delta\left(p_{1}=0\right)$. So the decay of the one-particle correlation function will be determined by this quantity. The proof of the existence of a line of bound states in the two-particle sector is also straightforward. By definition a bound state between two particles, 1 and 2 say, exists when their momenta acquire an imaginary part. We see from equation (4.8) that such an imaginary part must be included in the phase shift $\psi_{p_{1} p_{2}}$ and since we are interested in the thermodynamic limit we therefore put $\psi_{p_{1} p_{2}}=\mathrm{i} \phi L$. Hence $p_{1}=\frac{2 \pi}{L} n_{1}+i \phi$ and $p_{2}=\frac{2 \pi}{L} n_{2}-i \phi$. Substituting these two equations in (4.9) with $\psi_{p_{1} p_{2}}$ of the above form one obtains

$$
\frac{(1+\delta-\alpha-\gamma) \sin \left[\frac{\pi}{L}\left(n_{1}-n_{2}\right)+i \phi\right]}{\cos \left[\frac{\pi}{L}\left(n_{1}+n_{2}\right)\right]-(1+\delta-\alpha-\gamma) \cos \left[\frac{\pi}{L}\left(n_{1}-n_{2}\right)+i \phi\right]}=\cot \left(\frac{i L \phi}{2}\right) \rightarrow \mp i
$$

for $L$ large. The minus (plus) sign applies when $\phi$ is positive (negative), and we have substituted $h_{11}$, $h_{22}, h_{33}, h_{44}, h_{23}$ and $h_{32}$ by their values as given in (2.21). We shall from now on, and as in section 
2, use the abbreviation $\Delta=1+\delta-\alpha-\gamma$. We can, after some trivial manipulations write (E.1) as $\cos \left[\frac{\pi}{L}\left(n_{1}+n_{2}\right)\right]=\Delta e^{-|\phi|} e^{ \pm i \frac{\pi}{L}\left(n_{1}-n_{2}\right)}$. From this equation it follows that $n_{1}-n_{2}=k \pi$ where $k$ is an integer. It also follows that if $|\Delta|<1$ then the maximum value $\cos \left[\frac{\pi}{L}\left(n_{1}+n_{2}\right)\right]$ can achieve is $|\Delta|<1$. So there is no bound state with zero total momentum within this range of $\Delta$. The energy of the bound state can be calculated from (4.5) after the appropriate substitutions and the use of the cosine addition formula and is equal to

$$
E_{b}=4(1+\delta)-2 \Delta-\frac{2}{\Delta} \cos ^{2}\left[\frac{\pi}{L}\left(n_{1}+n_{2}\right)\right]
$$

Now one can ask when this energy will be lower than the lowest lying state in the continuum namely the state with energy $E=4 \delta\left(p_{1}=p_{2}=0\right)$. This will happen if $4-2\left(\Delta+\frac{1}{\Delta} \cos ^{2}\left[\frac{\pi}{L}\left(n_{1}+n_{2}\right)\right]\right)<0$ subjected to the restriction $\cos \left[\frac{\pi}{L}\left(n_{1}+n_{2}\right)\right]=(-1)^{k} \Delta e^{-|\phi|}$. From these equations one obtains $\Delta>1$ and $E=4 \delta-2\left(\Delta-2+\Delta^{-1}\right)$ for the energy of the lowest lying state in the line of bound states $\left(n_{1}=-n_{2}\right)$. So for this range of the parameter the long time behaviour of two point correlation functions will be determined by the energy of this state. For the other values of $\Delta$ the decay is determined by the state in the continuum with lowest energy $(E=4 \delta)$. These are the results presented in Table 2.

Let us now show how these results arise in the context of the equations of motion for the correlation functions, following [10]. First let us consider the one-point correlation function. The equation of motion for the expectation value is obtained by using the Heisenberg equation of motion $\frac{d}{d t}\left\langle s\left|\tilde{n}_{j}(t)\right| \Psi\right\rangle=$ $\left\langle s\left|\left[H, \tilde{n}_{j}(t)\right]\right| \Psi\right\rangle$ where $H$ is given by (2.21) and we are taking the average in the initial state $|\Psi\rangle$ in the way indicated by (2.7). Using the properties of $\langle s|$ one obtains

$$
\begin{aligned}
\frac{d}{d t}<\tilde{n}_{j}(t)>= & <\tilde{n}_{j+1}(t)>+<\tilde{n}_{j-1}(t)>-2(1+\delta)<\tilde{n}_{j}(t)> \\
& +(\delta-\gamma-2 \alpha)\left(<\tilde{n}_{j}(t) \tilde{n}_{j+1}(t)>+<\tilde{n}_{j-1}(t) \tilde{n}_{j}(t)>\right)
\end{aligned}
$$

where we are using the abbreviation $\left\langle\tilde{n}_{j}(t)\right\rangle=\left\langle s\left|\tilde{n}_{j}(t)\right| \Psi\right\rangle$. Now one knows that the energy of the two particle lowest lying state (either the state with $p_{1}=p_{2}=0$ for $\Delta \leq 1$ or the bound state for $\Delta>1$ ) is higher than the energy of the lowest lying state of the one particle sector (which has an energy equal to $\left.E=2 \delta\left(p_{1}=0\right)\right)$. So the last term of the r.h.s of $(\mathbb{E} .3)$ has a faster decay than the remaining ones since the decay of this term is determined by a higher value of the energy. Hence we can drop it at large times [10]. Assuming a translationally invariant initial state with $<n_{j}(0)>=\rho_{0}$, one finds for the average particle density at $t$ large $<\tilde{n}_{j}(t)>=\rho_{0} e^{-2 \delta t}$, which is the result presented in Table 2. The equation for the two point correlation function is obtained in a similar way. Taking the 
expectation value of the Heisenberg equation of motion for $\tilde{n}_{j}(t) \tilde{n}_{l}(t)(l>j)$ one obtains

$$
\begin{aligned}
\frac{d}{d t}<\tilde{n}_{j}(t) \tilde{n}_{l}(t)>= & <\tilde{n}_{j+1}(t) \tilde{n}_{l}(t)>+<\tilde{n}_{j-1}(t) \tilde{n}_{l}(t)>+<\tilde{n}_{j}(t) \tilde{n}_{l-1}(t)> \\
& +<\tilde{n}_{j}(t) \tilde{n}_{l+1}(t)>-4(1+\delta)<\tilde{n}_{j}(t) \tilde{n}_{l}(t)> \\
& +(\delta-\gamma-2 \alpha)\left(<\tilde{n}_{j}(t) \tilde{n}_{j+1}(t) \tilde{n}_{l}(t)>+<\tilde{n}_{j-1}(t) \tilde{n}_{j}(t) \tilde{n}_{l}(t)>\right. \\
& \left.+<\tilde{n}_{j}(t) \tilde{n}_{l}(t) \tilde{n}_{l+1}(t)>+<\tilde{n}_{j}(t) \tilde{n}_{l-1}(t) \tilde{n}_{l}(t)>\right)
\end{aligned}
$$

for $l \neq j+1$ and

$$
\begin{aligned}
\frac{d}{d t}<\tilde{n}_{j}(t) \tilde{n}_{j+1}(t)>= & <\tilde{n}_{j-1}(t) \tilde{n}_{j}(t)>+<\tilde{n}_{j}(t) \tilde{n}_{j+2}(t)>-2(1+\delta+\gamma+\alpha)<\tilde{n}_{j}(t) \tilde{n}_{j+1}(t)> \\
& +(\delta-\gamma-2 \alpha)\left(<\tilde{n}_{j}(t) \tilde{n}_{j+1}(t) \tilde{n}_{j+2}(t)>+<\tilde{n}_{j-1}(t) \tilde{n}_{j}(t) \tilde{n}_{j+1}(t)>\right)(\mathrm{E} .5)
\end{aligned}
$$

for $l=j+1$. Now one proceeds as before. It is easy to see that there is no bound state involving three particles in the three particle sector. This is so because then all the momenta of the three particles would have an imaginary part and the energy of the state would be complex. This is impossible given that $H_{X X Z}$, which determines the spectrum, is hermitian. So the energy of the lowest lying state in the three particle sector is higher than the energy of the lowest lying state in the two particle sector. Therefore the three particle correlators decay faster then the two-particle correlators and can at large times be dropped from equations $(\mathbb{E . 4}$ and $(\mathbb{E} .5)$. If we assume, as above a translational invariant initial state then the truncated equations for the two-point correlation function become, where $C(r, t)=<\tilde{n}_{i}(t) \tilde{n}_{i+r}(t)>$

$$
\frac{d}{d t} C(r, t)=-4(1+\delta) C(r, t)+2(C(r+1, t)+C(r-1, t))
$$

for $r \neq 1$ and

$$
\frac{d}{d t} C(1, t)=-2(1+\delta+\gamma+\alpha) C(1, t)+2 C(2, t)
$$

for $r=1$. Now equation ( $\mathbb{E} .7)$ can be given the form (E.6) if we define $C(0, t)=\Delta C(1, t)$. So now we have to solve equation (E.6) with this boundary condition. The solution is

$$
C(r, t)=e^{-4(1+\delta) t} \sum_{y=1}^{\infty}\left[a_{y} I_{r-y}(4 t)+b_{y} I_{r+y-1}(4 t)\right]
$$

where $I_{n}(4 t)$ is the modified Bessel function of order $n$, the $a_{y}$ 's are constants defined by the initial distribution $\square$ and $b_{y}=\Delta a_{y}-\left(1-\Delta^{2}\right) \sum_{k=1}^{y-1} \Delta^{y-1-k} a_{k}$. We substitute this in (E.8) and interchange

\footnotetext{
${ }^{16}$ If $\delta=2 \alpha+\gamma$, eqs. (E.6, E.7) are exact at all times. Then the $a_{y}$ are given by the initial conditions of the full physical problem. Otherwise, this relation is considerably more complicated.
} 
the sums between $y$ and $k$. We get after a few manipulations

$$
\begin{aligned}
C(r, t)= & e^{-4(1+\delta) t} \sum_{y=1}^{\infty}\left[a_{y} I_{r-y}(4 t)+\Delta a_{y} I_{r+y-1}(4 t)\right] \\
& -\left(1-\Delta^{2}\right) e^{-4(1+\delta) t} \sum_{k=1}^{\infty} a_{k} \Delta^{-k} \sum_{y=-\infty}^{+\infty} I_{y+r-1}(4 t) \Delta^{y-1} \\
& +\left(1-\Delta^{2}\right) e^{-4(1+\delta) t} \sum_{k=1}^{\infty} a_{k} \sum_{y=-\infty}^{0} I_{y+r+k-1}(4 t) \Delta^{y-1}
\end{aligned}
$$

where we have completed the sum in $y$ in the second term and accordingly subtracted the third term. Since $e^{-4 t} I_{n}(4 t) \leq 1$ then the sum over $y$ in the third term is convergent if $|\Delta|>1$. Hence, provided that the sum over $a_{k}$ is convergent this term is bounded by $\left(1-\Delta^{2}\right) e^{-4 \delta t} \frac{\left|\sum_{k=1}^{\infty} a_{k}\right|}{|\Delta-1|}$ when $|\Delta|>1$. One can then explicitly perform the sum over $y$ in the second term, using the generating function for modified Bessel functions. Hence if $|\Delta|>1$ one gets

$$
\begin{aligned}
C(r, t)= & e^{-4(1+\delta) t} \sum_{y=1}^{\infty}\left[a_{y} I_{r-y}(4 t)+\Delta a_{y} I_{r+y-1}(4 t)\right] \\
& -\left(1-\Delta^{2}\right) \Delta^{-r} e^{-\left[4 \delta-2\left(\Delta-2+\Delta^{-1}\right)\right] t} \sum_{k=1}^{\infty} a_{k} \Delta^{-k} \\
& +\left(1-\Delta^{2}\right) e^{-4(1+\delta) t} \sum_{k=1}^{\infty} a_{k} \sum_{y=-\infty}^{0} I_{y+r+k-1}(4 t) \Delta^{y-1}
\end{aligned}
$$

One sees that at long times the first and third terms of this equation decay like $e^{-4 \delta t}$. Hence if $\Delta>1$ the decay of the two-point correlation functions is dominated by the second term and the long time behaviour of the two point correlation function would be proportional to $e^{-\left[4 \delta-2\left(\Delta-2+\Delta^{-1}\right)\right] t}$. Also we see that the second term does not appear if $|\Delta|<1$, which is in agreement with the result obtained by the Bethe Ansatz that there is no bound state with total zero momenta in this range of the parameter $\Delta$, otherwise such state would appear in the decay of correlation functions from a translational invariant state. Hence we again obtain the results of Table 2.

\section{Appendix F. Role of the boundary conditions on the Hamilto- nian spectrum}

We discuss the relationship between the quantum Hamiltonian spectra for free and periodic boundary conditions. The main point already becomes apparent when just considering asymmetric diffusion without any further reactions [21, 47, 27]. While this problem is still very technical for the case of a finite particle density, the basic mechanism becomes apparent in a simple way already for a finite 
number of particles. To make this paper more self-contained, we recall the argument here, following 477.

For free boundary conditions on a chain of $L$ sites, the quantum Hamiltonian reads

$$
H^{\prime}=-\frac{1}{4 \Delta} \sum_{j=1}^{L-1}\left[\sigma_{j}^{x} \sigma_{j+1}^{x}+\sigma_{j}^{y} \sigma_{j+1}^{y}+\Delta \sigma_{j}^{z} \sigma_{j+1}^{z}-\frac{q-q^{-1}}{2}\left(\sigma_{j}^{z}-\sigma_{j+1}^{z}\right)-\Delta\right]
$$

where

$$
\Delta=\frac{q+q^{-1}}{2}, q=\sqrt{\frac{1-\epsilon}{1+\epsilon}}
$$

This is the well-known XXZ quantum chain which is symmetric under the quantum group $U_{q} S U(2)$ [45, 46]. It follows from the Bethe ansatz that for $\epsilon \neq 0$ the energies for $L$ large become $E_{L}{ }^{\prime} \sim 1-\Delta^{-1}$ in each sector $[7$ with $N$ particles (see also below). We stress that the quantum Hamiltonian of the diffusion process on a periodic lattice cannot be obtained by simply taking periodic boundary conditions in eq. (F.1). Rather, from the master equation $H$ can be written down directly [21]

$$
H=-\frac{1}{4} \sum_{j=1}^{L}\left[\vec{\sigma}_{j} \cdot \vec{\sigma}_{j+1}-1+i \epsilon\left(\sigma_{j}^{x} \sigma_{j+1}^{y}-\sigma_{j}^{y} \sigma_{j+1}^{x}\right)\right]
$$

where $\sigma^{x, y, z}$ are Pauli matrices and $\epsilon$ is related to the diffusion bias via equation (F.2). It is well known that the low-lying spectrum of $H$ is gapless (see also below).

To understand the relation between $H$ and $H^{\prime}$, we introduce $\sigma^{ \pm}=\frac{1}{2}\left(\sigma^{x} \pm i \sigma^{y}\right)$, and consider the non-singular operator

$$
U=\exp \left(\pi g \sum_{j=1}^{L} j \sigma_{j}^{z}\right) \quad, U \sigma_{j}^{ \pm} U^{-1}=e^{ \pm 2 \pi g j} \sigma_{j}^{ \pm}
$$

Choosing $q=e^{2 \pi g}$, we obtain

$$
H^{\prime \prime}=U H U^{-1}=-\frac{1}{2\left(q+q^{-1}\right)} \sum_{j=1}^{L}\left[\sigma_{j}^{x} \sigma_{j+1}^{x}+\sigma_{j}^{y} \sigma_{j+1}^{y}+\Delta \sigma_{j}^{z} \sigma_{j+1}^{z}-\Delta\right]
$$

which is indeed (almost) the Hamiltonian $H^{\prime}$. The distinction comes from the boundary conditions. The surface field $\left(q-q^{-1}\right)\left(\sigma_{1}^{z}-\sigma_{L}^{z}\right) / 8 \Delta$ is absent in $H^{\prime \prime}$ and one has

$$
\sigma_{L+1}^{ \pm}=q^{\mp L} \sigma_{1}^{ \pm}, \sigma_{L+1}^{z}=\sigma_{1}^{z}
$$

which make $H^{\prime \prime}$ non-hermitian, as $H$ already is. It is these unusal boundary conditions which give rise to the different properties of $H$ and $H^{\prime}$, as we now show.

\footnotetext{
${ }^{17}$ Since $H^{\prime}$ commutes with the quantum group generators $S_{ \pm}$and $S_{z}$, for each state $|M\rangle$ in the $M$-particle sector with energy $E^{\prime}$ there a $(N+M)$-particle state $|N+M\rangle \sim S_{-}^{N}|M\rangle$ with the same energy, see [28].
} 
The calculation of the spectrum of $H^{\prime \prime}$ proceeds via the Bethe ansatz [69]. First, we consider the sector with $N=1$ particle. Then the energies are in this sector

$$
E=1-\Delta^{-1} \cos \theta, \quad \theta=2 \pi\left(i g+\frac{n}{L}\right)
$$

where $n$ is an integer from the set $\{0, \pm 1, \ldots, \pm(L / 2-1), L / 2\}$. For $L$ large, the energies become

$$
E=1-\frac{\cos \left(2 \pi\left(i g+\frac{n}{L}\right)\right)}{\cosh (2 \pi g)} \simeq 2 \pi^{2}\left(\frac{n}{L}\right)^{2}+2 \pi i \tanh (2 \pi g) \frac{n}{L}+\ldots
$$

We see that the real part $\Re E \sim L^{-2}$ and the imaginary part $\Im E \sim L^{-1}$.

Next, we take the sector $N=2$. From the Bethe ansatz [69] we have

$$
\begin{aligned}
& E=\left(2 \Delta-\cos \theta-\cos \theta^{\prime}\right) \Delta^{-1} \\
& \theta L-2 \pi i g L=2 \pi I-\Theta\left(\theta, \theta^{\prime}\right), \quad \theta^{\prime} L-2 \pi i g L=2 \pi I^{\prime}-\Theta\left(\theta^{\prime}, \theta\right)
\end{aligned}
$$

where $I, I^{\prime}$ are distinct half-integers from the set $\left\{ \pm \frac{1}{2}, \pm \frac{3}{2}, \ldots, \pm \frac{L-1}{2}\right\}$ and

$$
\Theta\left(\theta, \theta^{\prime}\right)=2 \arctan \left(\frac{\Delta \sin \left(\frac{1}{2}\left(\theta-\theta^{\prime}\right)\right)}{\cos \left(\frac{1}{2}\left(\theta+\theta^{\prime}\right)\right)-\Delta \cos \left(\frac{1}{2}\left(\theta-\theta^{\prime}\right)\right)}\right)
$$

Define $\widetilde{\theta}=\theta-2 \pi i g, \widetilde{\theta}^{\prime}=\theta^{\prime}-2 \pi i g$. Then, for small values of the arguments

$$
\Theta\left(\widetilde{\theta}, \widetilde{\theta^{\prime}}\right) \simeq 2 \arctan \left(i \operatorname{coth}(2 \pi g) \cdot \frac{\widetilde{\theta}-\widetilde{\theta}^{\prime}}{\widetilde{\theta}+\widetilde{\theta}^{\prime}}\right)
$$

which is of order unity. Therefore

$$
\widetilde{\theta}=\frac{2 \pi}{L} a, \quad \widetilde{\theta}^{\prime}=\frac{2 \pi}{L} a^{\prime}
$$

where $a, a^{\prime}$ are of order $\mathcal{O}(1)$. It follows that the same cancellation as observed in the sector $N=1$ also takes place here and also that the observed scaling of the energies does not change. Finally, for $N$ arbitrary

$$
E=\Delta^{-1}\left(N \Delta-\sum_{n=1}^{N} \cos \theta_{n}\right)
$$

with

$$
\theta_{m} L-2 \pi i g L=2 \pi I_{m}-\sum_{n=1}^{N} \Theta\left(\theta_{m}, \theta_{n}\right)
$$

and the same argument can be repeated. It follows that for any value of $\epsilon$, the spectrum shows in the $L \rightarrow \infty$ limit no energy gap.

While this argument shows that for a finite number of particles, the real part of the energies scales as $L^{-2}$, a much more detailed analysis of the Bethe ansatz equations shows [21, 27 that for a finite density, 
one rather has a scaling $\Re E \sim L^{-3 / 2}$. This arguments also goes through when particle reactions are added [47]. In the case of more than one species of particles, the relation between $H$ and $H^{\prime}$ becomes even more involved, as explained in [26].

A simple interpretation of this result is as follows. For periodic boundary conditions, the particles are free to chase each other. In fact, through a Galilei transformation on can go into the frame where the diffusion becomes left-right symmetric, see [70]. It is not surprising that it should take a long time for the system to reach a steady state (in fact, $\Im E$ is proportional to the steady state current density) and that the relaxation time should grow beyond all bounds with increasing system size $L$. On the other hand, for free boundary conditions the particles cannot leave the system. Rather, they pile up on one of the boundaries. The time to reach a steady state is of the order of the time a single particle needs to cross the system from one boundary to the other and should be finite. 


\section{References}

[1] J. Marro and R. Dickman, "Nonequilibrium phase transitions in lattice models", Cambridge University Press, Cambridge, 1997.

[2] V. Privman (ed.) "Nonequlibrium Statistical Mechanics in One Dimension", Cambridge University Press, Cambridge, 1996.

[3] A. McKane, M. Droz, J. Vannimenus and D. Wolf (Eds), "Scale Invariance, Interfaces and Non-Equilibrium Dynamics", NATO-ASI Vol. B344, Plenum Press, New York, 1995.

[4] B. Schmittmann and R.K.P. Zia, in "Phase Transitions and Critical Phenomena" (C. Domb and J. Lebowitz Eds.), Vol. 17, Academic Press, London, 1995.

[5] M. Droz, in "Diffusion processes: Experiments, theory, simulations", Springer Lecture Notes in Physics, Vol. 438, p. 105-114, Springer Verlag, Heidelberg, 1995.

[6] D. Tousaint and F. Wilczek, J. Chem. Phys. 78 (1983), 2642.

[7] J. Prasad and R. Kopelman, Chem. Phys. Lett. 157 (1989), 535.

[8] R. Kopelman, C. S. Li and Z.-Y. Shi, J. Luminescence 45 (1990), 40.

[9] R. Kroon, H. Fleurent and R. Sprik, Phys. Rev. E47 (1993), 2462.

[10] M. Henkel, E. Orlandini and G. M. Schütz, J. Phys. A28 (1995), 6335.

[11] M. V. Smoluchowski, Z. Phys. Chem. 92 (1917), 129.

[12] V. Kuzovkov and E. Kotomin, Rep. Prog. Phys. 51 (1988), 1479.

[13] J. L. Cardy, Renormalisation group approach to reaction-diffusion problems, Oxford preprint, condmat/9607163.

[14] R. J. Glauber, J. Math. Phys. 4 (1963), 294.

[15] M. Doi, J. Phys. A9 (1976), 1465.

[16] L. P. Kadanoff and J. Swift, Phys. Rev 165 (1968), 310.

[17] P. Grassberger and M. Scheunert, Fortschr. Phys. 28 (1980), 547.

[18] U. Felderhof, Rep. Math. Phys. 1 (1970), 219.

[19] S. Alexander and T. Holstein, Phys. Rev. B18 (1978), 301.

[20] D. Kandel, E. Domany and B. Nienhuis, J. Phys. A23 (1990), L755.

[21] L.-H. Gwa and H. Spohn, Phys. Rev. A46 (1992), 844.

[22] G. Schütz, J. Stat. Phys. 71 (1993), 471.

[23] F. C. Alcaraz and V. Rittenberg, Phys. Lett. B314 (1993), 377.

[24] F. C. Alcaraz, M. Droz, M. Henkel and V. Rittenberg, Ann. of Phys. 230 (1994), 250.

[25] G. M. Schütz, J. Stat. Phys. 79 (1995), 243.

[26] S. R. Dahmen, J. Phys. A28 (1995), 905. 
[27] D. Kim, Phys. Rev. E52 (1995), 3512.

[28] S. Sandow and G. Schütz, Europhys. Lett. 26 (1994), 7.

[29] H. Hinrichsen, S. Sandow and I. Peschel, J. Phys. A29 (1996) 2643.

[30] G.T. Barkema and G.M. Schütz, Europhys. Lett. 35 (1996), 139.

[31] V.I. Tokar, Phys. Rev. E53 (1996), 1411.

[32] S. Albeverio, S.-M. Fei, Symmetry, integrable chain model and stochastic processes, Bochum preprint, hep-th/9605130.

[33] G. Albertini, S. R. Dahmen and B. Wehefritz, Nucl. Phys. B493 (1997), 541.

[34] H. Hinrichsen, V. Rittenberg and H. Simon, J. Stat. Phys. 86 (1997), 1203.

[35] D.C. Mattis, "The Theory of Magnetism", Vol. 1, Springer Verlag, Berlin, 1988.

[36] M. Gaudin, "La fonction d'onde de Bethe", Masson, Paris, 1983.

[37] R. J. Baxter, "Exactly solved models in statistical mechanics", Academic Press, New York, 1982.

[38] K. Krebs, M. P. Pfannmüller, B. Wehefritz and H. Hinrichsen, J. Stat. Phys. 78 (1995), 1429.

[39] H. Simon, J. Phys. A28 (1995), 6585.

[40] V. Privman, A. M. R. Cadilhe and M. L. Glasser, J. Stat. Phys. 81 (1995), 881.

[41] R.B. Stinchcombe, M. Grynberg and M. Barma, Phys. Rev. E47 (1993), 4018.

[42] Z. Racz, Phys. Rev. Lett. 55 (1985), 1707.

[43] J. Santos, J. Phys. A 30 (1997), 3249.

[44] C. Hyver, J. Theor. Biol. 36 (1972), 133;

J. Keizer, J. Stat. Phys. 6 (1972), 67;

J. Schnakenberg, Rev. Mod. Phys. 48 (1976), 571.

[45] A.N. Kirrilov and N. Yu. Reshetikhin, LOMI preprint (1988).

[46] V. Pasquier and H. Saleur, Nucl. Phys. B330 (1990), 523.

[47] M. Henkel and G. M. Schütz, Physica A206 (1994), 187.

[48] I. Peschel, V. Rittenberg and U. Schultze, Nucl. Phys. B430 (1995), 633.

[49] J. D. Johnson and B. M. McCoy, Phys. Rev. A6 (1972), 1613.

[50] M. Takanishi, Prog. Theor. Phys. 50 (1973), 1519.

[51] V. Privman, Phys. Rev. A46 (1992), R6140.

[52] D. Balboni, P.-A. Rey and M. Droz, Phys. Rev. E52 (1995), 6220.

[53] C.N. Yang and C.P. Yang, Phys. Rev. 150 (1966), 321.

[54] D. ben-Avraham, M. A. Burschka and C. R. Doering, J. Stat. Phys. 60 (1990), 695;

J. L. Spouge, Phys. Rev. Lett. 60 (1988), 871;

F. Family and J. G. Amar, J. Stat. Phys. 65 (1991), 1235. 
[55] A. A. Lushnikov, Sov. Phys. JETP 64 (1986), 811; Phys. Lett. 120A (1987), 135.

[56] L. Peliti, J. Physique 46 (1985), 1469.

[57] R. Dickman, Phys. Rev. B40 (1989), 4005.

[58] M. Henkel, D. Karevski and J.F.F. Mendes, unpublished (1997).

[59] K. Kang and S. Redner, Phys. Rev. Lett. 52 (1984), 955.

[60] P.-A. Rey and M. Droz, J. Phys. A 30 (1997), 1101.

[61] M. D. Grynberg, T. J. Newman and R. B. Stinchcombe, Phys. Rev. E50 (1994), 957;

M.D. Grynberg and R.B. Stinchcombe, Phys. Rev. Lett. 74 (1995), 1242.

[62] R. Dickman and A. Yu. Tretyakov, Phys. Rev. E52 (1995), 3218.

[63] H. Hinrichsen, K. Krebs and I. Peschel, Z. Phys. B100 (1996), 105.

[64] E. Siggia, Phys. Rev. B16 (1977), 2319.

[65] F. Igloí, I. Peschel and L. Turban, Adv. Phys. 42 (1993), 683.

[66] H. Bethe, Z. Phys. 71 (1931), 205.

[67] E. Lieb, T. Schultz and D. Mattis, Ann. of Phys. 16 (1961), 407.

[68] G. M. Schütz, J. Phys. A28 (1995), 3405.

[69] F.C. Alcaraz, M.N. Barber and M.T. Batchelor, Ann. of Phys. 182 (1988), 280.

[70] M. Henkel and G.M. Schütz, Int. J. Mod. Phys. B8 (1994), 3487. 


\section{Figure captions}

Fig. 1 Test of the positivity of the reaction rates in the case when the transformation parameters $a$ and $Y$ are both real and $h^{2}>1$. In each subfigure, the shaded regions correspond to the domains where one of the following reaction rates is negative with (a) $\widetilde{\alpha}$ (b) $\widetilde{\gamma}$ (c) $\widetilde{\nu}$ and (d) $\widetilde{\sigma}$.

Fig. 2 Test of the positivity of the reaction rates in the case when $a$ and $Y$ are both real and $h=\frac{1}{2} \sqrt{3}$. Shaded regions correspond to one of the reaction rates being negative with (a) $\widetilde{\alpha}$ (b) $\widetilde{\gamma}$ (c) $\widetilde{\nu}$ and (d) $\tilde{\sigma}$.

Fig. 3 Test of the positivity of the reaction rates when the transformation parameters $A$ and $q$ are real and $h^{2}>1$. Shaded regions correspond to one of the reaction rates being negative with (a) $\widetilde{\alpha}(\mathrm{b})$ $\widetilde{\gamma}(\mathrm{c}) \widetilde{\nu}$ and $(\mathrm{d}) \widetilde{\sigma}$. 
(a)

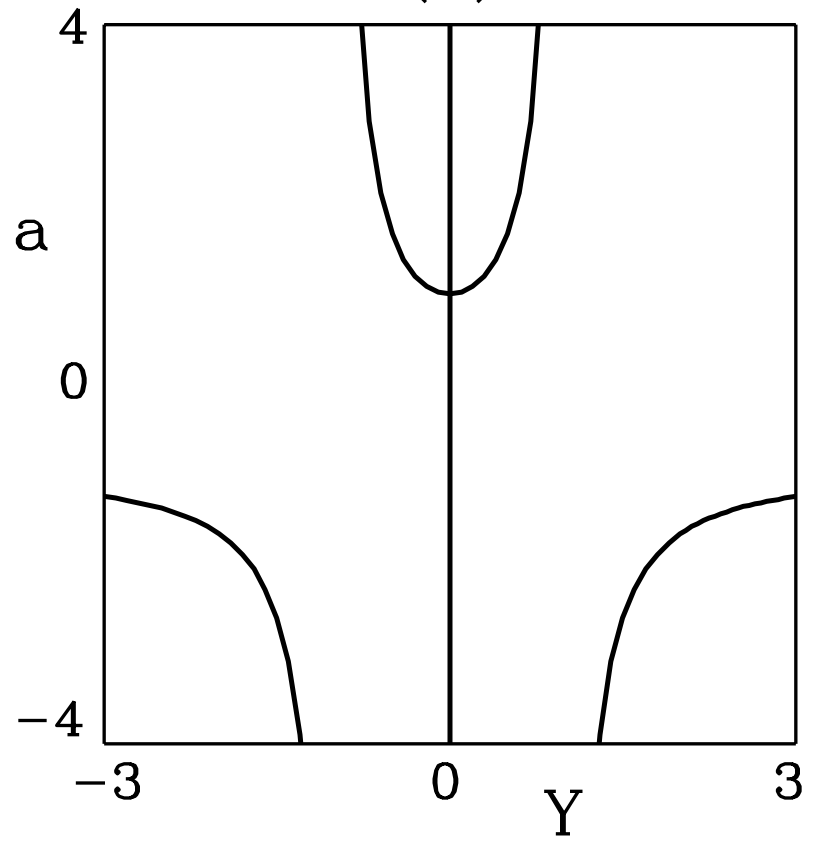

(c)

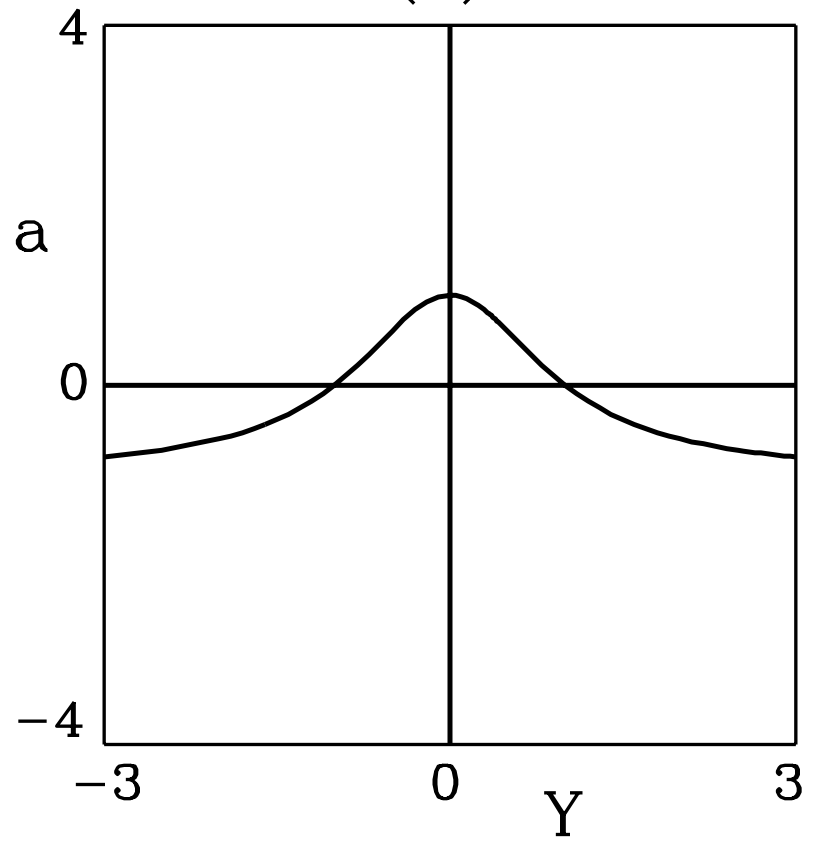

(b)

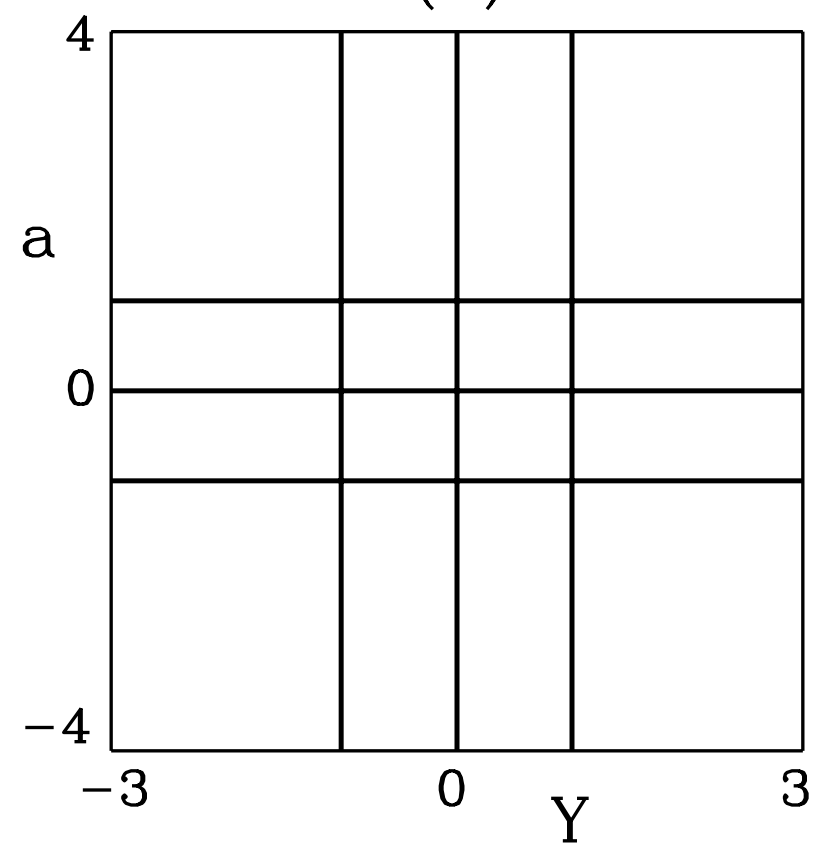

(d)

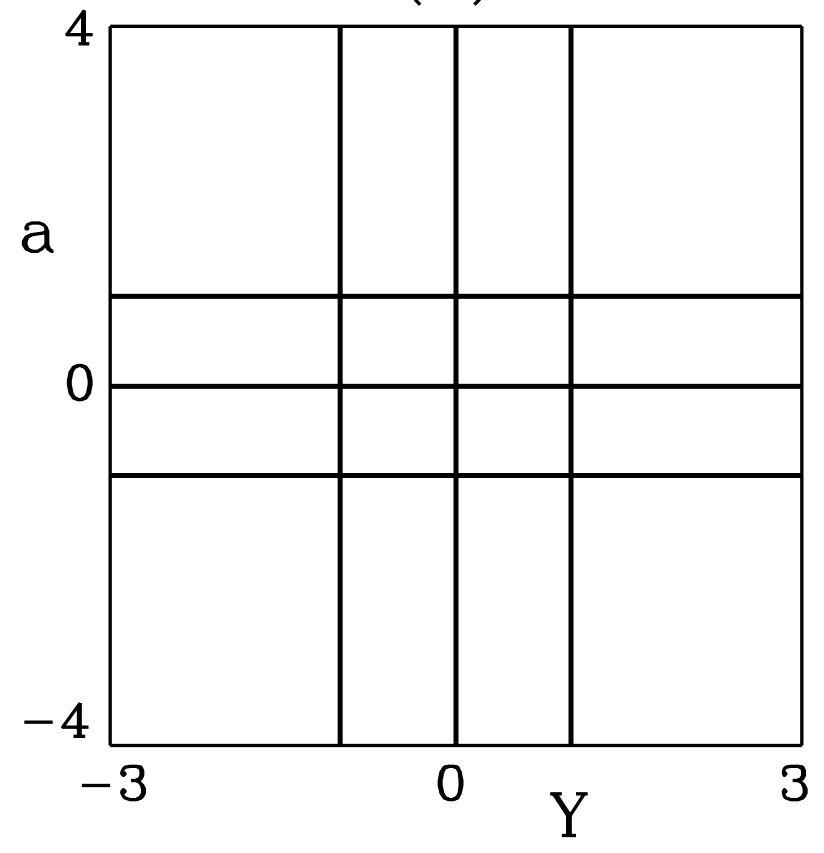


(a)

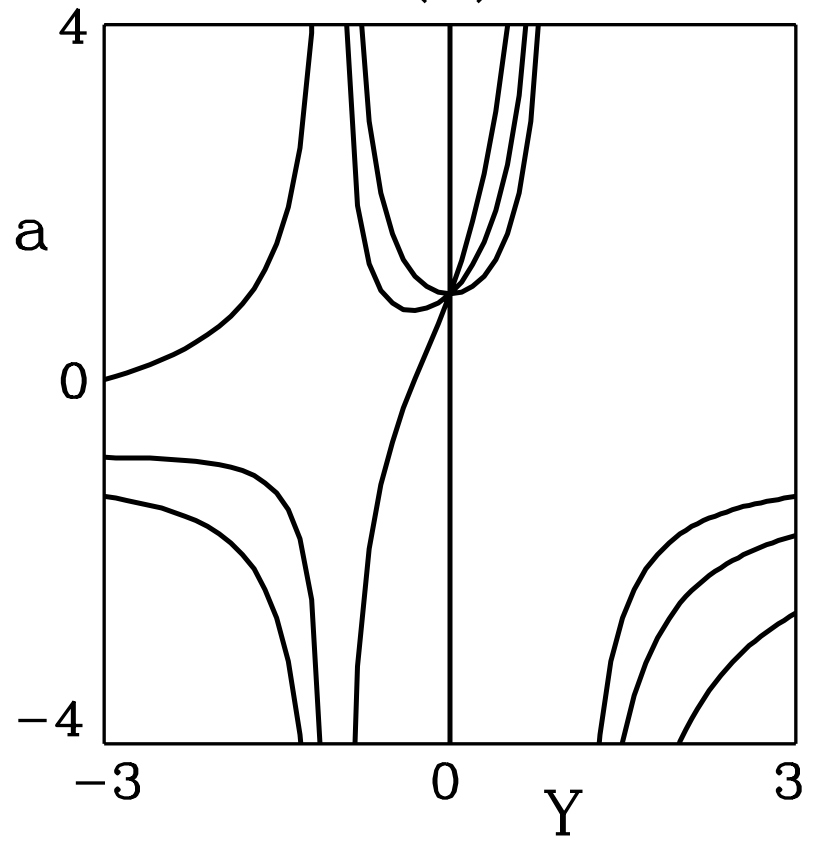

(c)

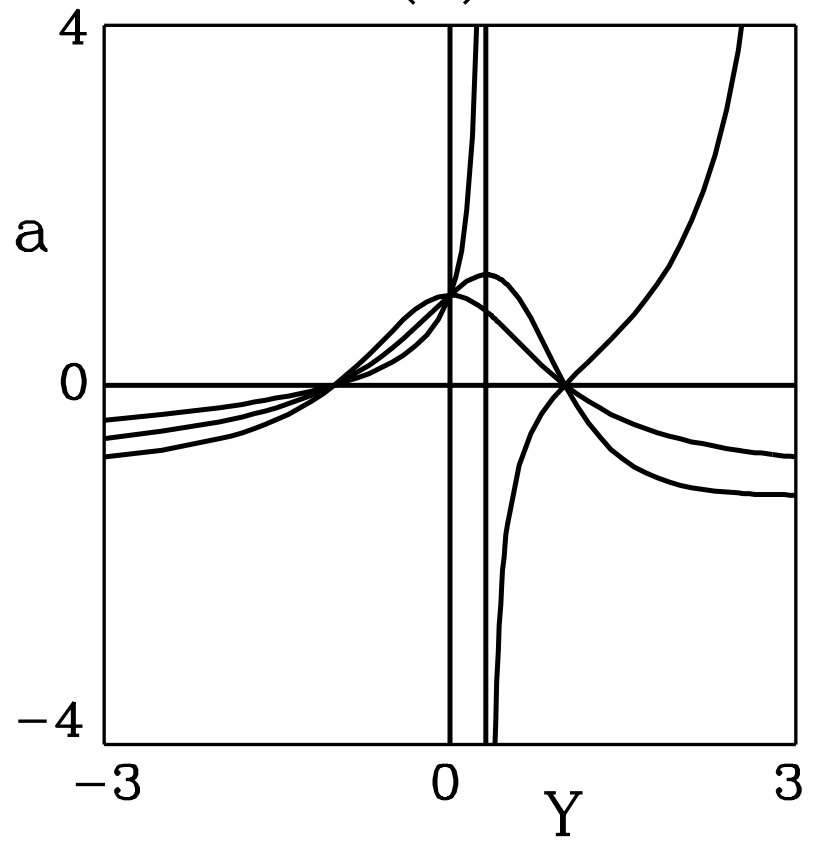

(b)

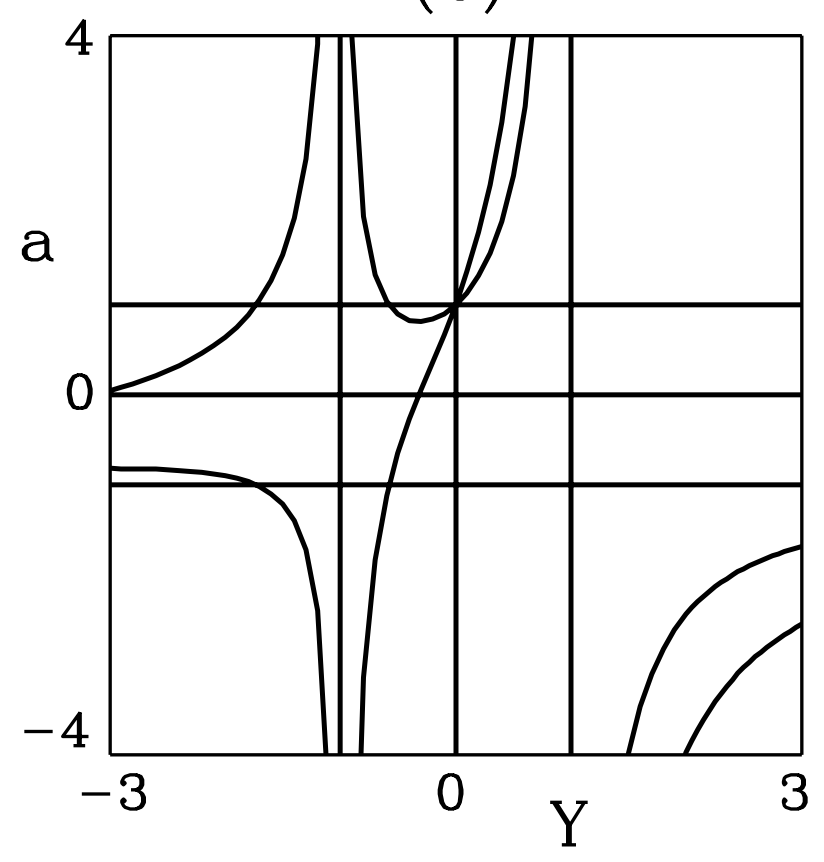

(d)

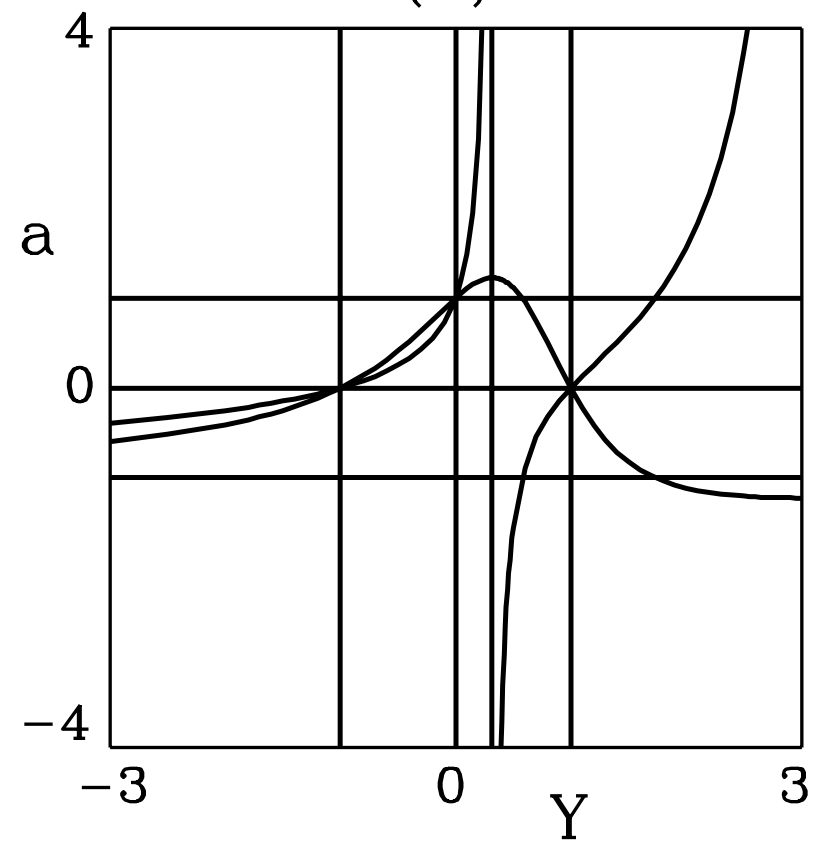



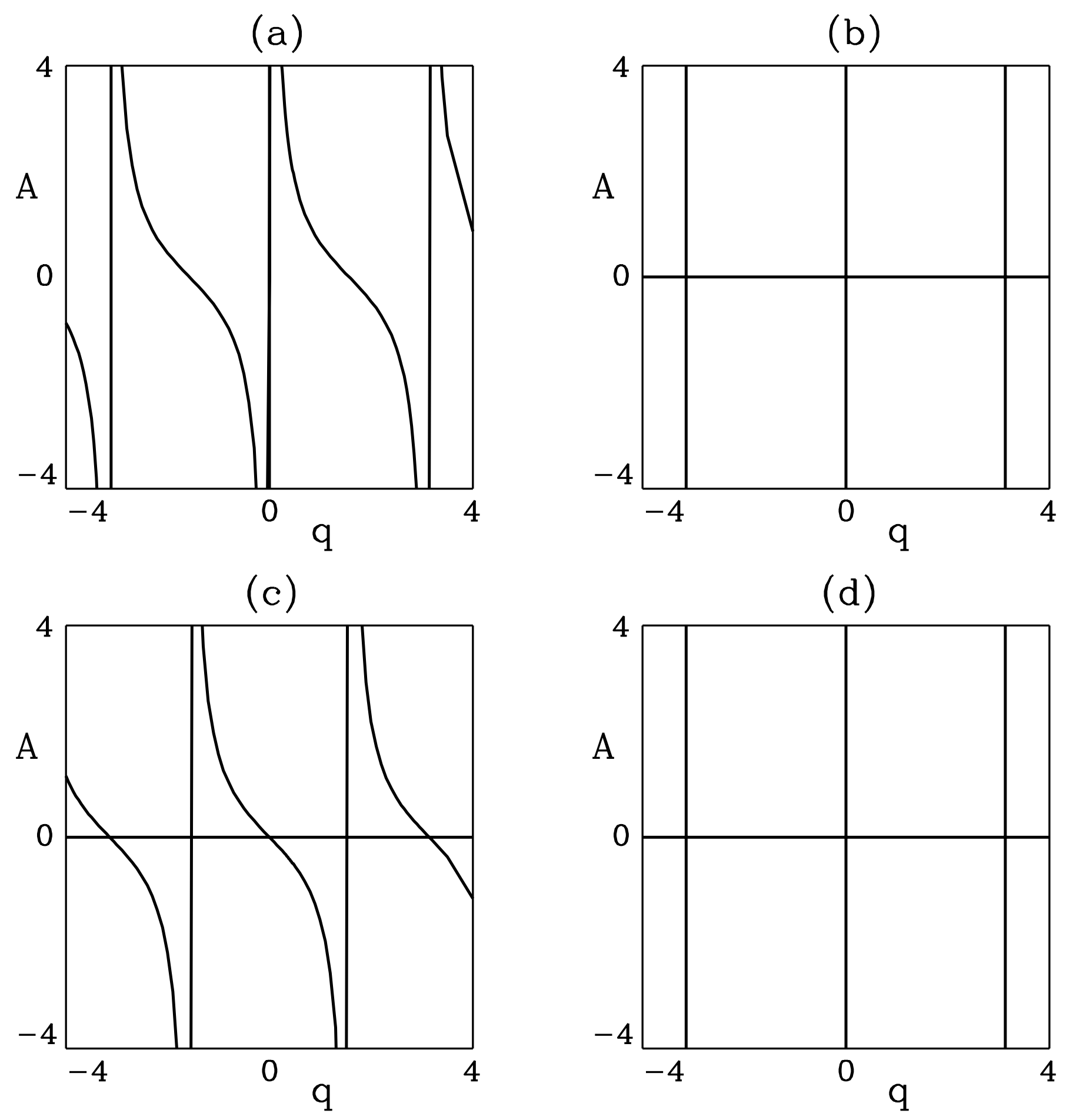